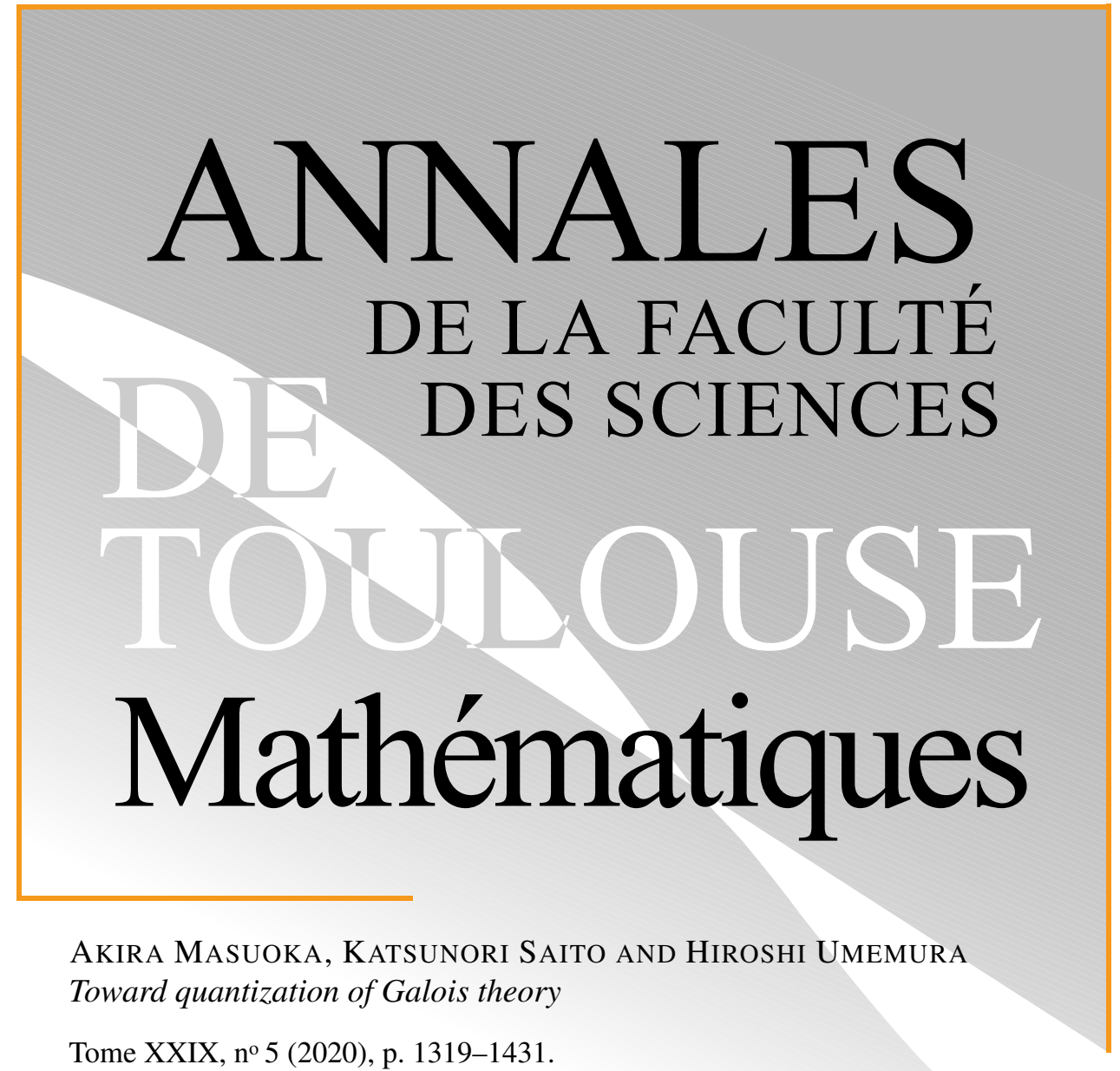

https://doi.org/10.5802/afst.1663

(C) Université Paul Sabatier, Toulouse, 2020.

L'accès aux articles de la revue «Annales de la faculté des sciences de Toulouse Mathématiques » (http://afst.centre-mersenne.org/) implique l'accord avec les conditions générales d'utilisation (http://afst.centre-mersenne.org/legal/). Les articles sont publiés sous la license CC-BY 4.0.

(c) $\underset{B Y}{(1)}$

Publication membre du centre

Mersenne pour l'édition scientifique ouverte MERSENNE http://www.centre-mersenne.org/ 


\title{
Toward quantization of Galois theory
}

\author{
Akira Masuoka ${ }^{(1)}$, Katsunori Saito ${ }^{(2)}$ And Hiroshi Umemura ${ }^{(3)}$
}

Abstract. - This article was born from our mathematical experiments, the first explorations of the unknown continent of quantized Galois theory. Known is classical Galois theory for linear differential equations, called Picard-Vessiot theory. Reformulating Picard-Vessiot theory in the language of Hopf algebras, we find a new way to a generalization, in which the commutative ring $\mathbb{C}[\mathrm{d} / \mathrm{d} x]$ of differential operators with constant coefficients is replaced by an arbitrary Hopf algebra of operators that may be non-commutative [1]. The Hopf algebras in this generalized theory are, however, basically assumed to be co-commutative since the theory is interested only in commutative rings with operators. Consequently their Galois groups are linear algebraic groups and the Galois theory is not quantized. Heiderich [1] combined the Hopf Galois theory [7] for linear equations with Umemura's general Galois theory [22] for non-linear differential equations.

In Parts I and II we use Heiderich's idea to investigate concrete examples of linear or non-linear difference-differential (or qsi) equations. In Part I we show through some examples that quantization of the Galois groups is indeed realized. The quantized Galois groups are Hopf algebras that are neither commutative nor co-commutative. In fact, quantization occurs even for linear equations. In Part II we investigate a special example of linear qsi equations to prove unique existence of the non-commutative Picard-Vessiot rings. These investigations of examples lead successfully to general results obtained in Part III, in which the examples are generalized to Hopf linear equations over a constant field $C$. The results are formulated in terms of arbitrary $C$-Hopf algebras $H$ and left $H$-modules of finite $C$-dimension, and they involve non-commutative Tannaka categories.

RÉSumÉ. - Cet article est né de nos expériences mathématiques, les premières explorations dans le continent inconnu de la théorie de Galois quantifiée. Nous connaissons la théorie de Galois classique des équations différentielles linéaires, appelée théorie de Picard-Vessiot. Quand la théorie de Picard-Vessiot est formulée dans le langage des algèbres de Hopf, elle ouvre une nouvelle voie vers la généralisation. Nous pourrions remplacer l'algèbre commutative $\mathbb{C}[\mathrm{d} / \mathrm{d} x]$ des opérateurs différentiels à coefficients constants par une algébre de Hopf d'opérateurs quelconque comme cela

(1) Institute of Mathematics, University of Tsukuba, Japan akira@math.tsukuba.ac.jp

Supported by JSPS KAKENHI Grant Number 17K05189.

(2) Graduate School of Mathematics, Nagoya University, Japan m07026e@math.nagoya-u.ac.jp

(3) Graduate School of Mathematics, Nagoya University, Japan — Hiroshi Umemura passed away on March 8, 2019. 
est fait dans [1]. Ces travaux s'intéressent uniquement aux anneaux commutatifs à opérateurs, les algèbres de Hopf dans cette théorie sont essentiellement supposées cocommutatives. Par conséquent, leurs groupes de Galois sont des groupes algébriques linéaires et la théorie de Galois n'est pas quantifiée.

Heiderich [7] a découvert que l'on peut réunir la théorie de Picard-Vessiot généralisée [1] et notre théorie de Galois [22] des équations différentielles non-linéaires. Nous appliquons son idée à certains exemples concrets et montrons, dans la première partie, que la théorie de Galois est bien quantifiée c'est-à-dire, le groupe de Galois qui est une algèbre de Hopf, n'est ni commutative, ni co-commutative, même pour les équations linéaires.

Dans la deuxième partie, nous analysons une équation différentielle et aux différences linéaire particulière. Nous montrons l'existence et l'unicité de l'extention de Picard-Vessiot non-commutative de l'équation.

L'analyse détaillée nous permet d'établir dans la troisième partie, pour une algèbre de Hopf quelconque d'opérateurs, la théorie de Galois quantique des équations linéaires à coefficients constants. Nous démontrons aussi l'équivalence des catégories tannakiennes non-commutatives. Ainsi donc, pour toute algèbre de Hopf $H$ sur un corps $C$ et tout $H$-module à gauche $M$ qui soit un espace $C$-vectoriel de dimension finie, nous avons la théorie de Galois exprimée en terme d'algèbres de Hopf.

\section{Introduction}

\subsection{Quantization of Galois theory}

The pursuit of $q$-analogue of hypergeometric series goes back to Heine [8], 1846. However, the Galois group of $q$-hypergeometric series is not a quantum group but it is a linear algebraic group. This shows that so far as we consider the $q$-analogue of the hypergeometric equation according to Heine, Galois theory is not quantized. In fact, generally we know that the Galois group of a linear difference equation is a linear algebraic group. So we may as well consider that Heine's $q$-hypergeometric series would be unsatisfactory as a quantization. To be more precise, the following question comes into our mind.

QUESTION 1.1. - Does there exist a thorough quantization of hypergeometric series in such a way that Galois groups of quantized series are general quantum groups?

More generally, we wonder if there would be a quantized Galois theory.

We owe Question 1.1 to Y. André [2] who first studied linear differencedifferential equations in the framework of non-commutative geometry. He encountered only linear algebraic groups. Later, Hardouin [5] also studied Picard-Vessiot theory of $q$-skew iterative $\sigma$-differential field extensions but 
also in this theory, the Galois group is a linear algebraic group. We clarified the situation in [26]. Namely, so far as we study linear difference-differential equations, however twisted or non-commutative the ring of difference and differential operators might be, Galois group, according to general Hopf Galois theory, is a linear algebraic group.

All the attempts of answering affirmatively Question 1.1 had been so far failed. Our results settle the Question 1.1 for linear equations with constant coefficients. See Part III. Question 1.1 is vague and we have to start by clarifying the nature of Question 1.1.

\subsection{What is $q$ ?}

In alphabet we have 26 letters. That is surely a small set if we compare it with the huge set of Chinese characters. It would be certainly by chance that the letter $q$ appears often in mathematics in different contexts.

(1) $q$ represents the $q$-analogue studied by L. Euler [4] and E. Heine [8] and many mathematicians since the last century.

(2) $q$ represents the number $q=p^{n}$ of elements of the finite field $\mathbb{F}_{q}=$ $\mathbb{F}_{p^{n}}$, where $p$ is a prime number.

(3) $q$ represents the variable $q=e^{2 \pi i \tau}$ in which modular functions are expanded as Laurent series; $\tau$ ranges over the upper-half plane.

(4) $q$ represents quantum physics, that is, non-commutative geometry.

These subjects were not a priori logically supposed to be related. However, there are surprising mathematical relations. For example, Euler [4] proved the pentagonal identity in 1740 which belongs to (1) and Jacobi [9] showed Euler's identity is a consequence of the triple product formula, revealing an unexpected relation between (1) and (3). So it is important to discover surprising relations among the subjects. Question 1 asks if there exist a Galois theoretic relations between (1) and (4).

\subsection{Our solution to Question 1}

In Part II, we explain our method of quantization of Picard-Vessiot extension by one of the simplest examples, which we generalize in Part III. Let $q \neq 0$ be a complex number with $q^{n} \neq 1$ for every positive integer $n$. Let $t$ be a variable over $\mathbb{C}$. We consider a difference-differential field or in our 
terminology a $q$ si field $\left(\mathbb{C}(t), \sigma^{ \pm 1}, \theta\right)$ so that $\sigma^{ \pm 1}: \mathbb{C}(t) \rightarrow \mathbb{C}(t), t \mapsto q^{ \pm 1} t$ are field automorphisms over $\mathbb{C}$ and $\theta: \mathbb{C}(t) \rightarrow \mathbb{C}(t)$ sends $f(t) \in \mathbb{C}(t)$ to

$$
\frac{f(q t)-f(t)}{(q-1) t} \text {. }
$$

We wonder, what is the Galois group of the qsi field extension $\left(\mathbb{C}(t), \sigma^{ \pm 1}, \theta\right) / \mathbb{C}$.

However, the extension is not, a Galois extension in any traditional sense. So we can not naively speak of its Galois group. (A qsi field is an abbreviation for a $q$-skew iterative $\sigma$-differential field. See Definition 3.5.)

Given a differential field extension $L / K$, the general Galois theory of Umemura [22, 23] and its generalization by Heiderich [7] construct a normalization $\mathcal{L} / \mathcal{K}$ of $L / K$. The construction, applied to the difference-differential field extension $\left(\mathbb{C}(t), \sigma^{ \pm 1}, \theta\right) / \mathbb{C}$ under some appropriate modification, produces a normalization of the qsi field extension $\left(\mathbb{C}(t), \sigma^{ \pm 1}, \theta\right) / \mathbb{C}$.

We have an inclusion $M:=\mathbb{C} .1+\mathbb{C} . t \subset \mathbb{C}(t)$ of difference-differential modules and the sub-field of $\mathbb{C}(t)$ generated by the qsi module $M$ coincides with the field $\mathbb{C}(t)$. So we could imagine from the analogous differential inclusion morphism $(\mathbb{C} .1+\mathbb{C} . t, \mathrm{~d} / \mathrm{d} t) \rightarrow(\mathbb{C}(t), \mathrm{d} / \mathrm{d} t) / \mathbb{C}$, the Galois group of the qsi extension coincides with the Galois group of the qsi module $M$.

The latter is a quantized Picard-Vessiot group. So we have to determine the Picard-Vessiot group of the qsi module $M$. We can show there exists a non-commutative Picard-Vessiot ring $R / \mathbb{C}$ of the difference-differential module $M$ :

(1) The difference-differential ring $R$ trivializes the difference-differential module $M$.

(2) The difference-differential ring $R$ is simple, i.e., there is no nontrivial bilateral invariant ideal of $R$.

(3) There exists a Hopf algebra $H$ such that the rigid tensor category of right $H$-co-modules of finite type is equivalent to the rigid tensor category $\{\{M\}\}$ generated by the difference-differential module $M$. These properties characterize the Hopf algebra $H$ and the noncommutative Picard-Vessiot extension $R / \mathbb{C}$. So the Hopf algebra $H$ that is neither commutative nor co-commutative, is the quantum Galois group of the difference-differential module $M$.

By the same idea we get generalized Picard-Vessiot extensions for qsi linear equations.

Alternatively, it is possible, given an $M$ as above, first to construct the Hopf algebra $H$ which has the property (3) above, and then to show that 
this $H$ itself acts as a non-commutative Picard-Vessiot ring, satisfying (1) and (2) above. A main idea is to present difference-differential modules as modules over an appropriate Hopf algebra, $\mathcal{H}_{q}$. This will be accomplished in Part III for an arbitrary Hopf algebra instead of the special $\mathcal{H}_{q}$.

\subsection{The composition of the paper}

The paper consists of Introduction, Parts I, II and III. Part I is a prospective part; we first review a general Galois theory for differential equations, difference equations and Hopf Galois theory, and then consider through examples what the Galois group of a qsi extension field should be. In fact we will describe explicitly the formal completions of such Galois groups for the following three examples of qsi extension fields with the constant field $\mathbb{C}$ :

(i) $\mathbb{C}(t) / \mathbb{C}$ in Section 4 .

(ii) $\mathbb{C}\left(t, t^{\alpha}\right) / \mathbb{C}$ in Section 5 .

(iii) $\mathbb{C}(t, \log t) / \mathbb{C}$ in Section 6 .

The first example reduces to the simplest linear qsi equation explained in the beginning of 1.3. It is a conjecture that the other two are not reducible to a linear qsi equation and have a huge Galois groups. In Part II, we study qsi equations and we give examples of quantized Picard-Vessiot extensions. In Part III we give Hopf-algebraic interpretations to some of the results obtained so far. The results will be proved for any finite-type module $M$ over an arbitrary Hopf algebra. The main idea is to construct for a given $M$, the Hopf algebra $H$ which has the property (3) above, and then to show that this $H$ and some generalized objects as well satisfy (1) and (2) above.

Throughout we work over a constant field $C$. In Parts I and II the characteristic of $C$ is assumed to be zero, for simplicity; also, the assumption is natural when we refer therein to the classical situation for differential equations. The assumption turns out, however, to be inessential in Part III, in which the characteristic is assumed to be arbitrary. We consider $C$-algebras. Except for Lie algebras, all the rings or algebras are associative $C$-algebras and contain the identity element. So the field $C$ is in the center of the algebras. Morphisms between them are unitary $C$-morphisms. For a commutative algebra $A$, we denote by $(\mathrm{Alg} / A)$ the category of commutative $A$-algebras, which we sometimes denote by $(\mathrm{CAlg} / A)$ to emphasize that we are dealing with commutative $A$-algebras. In fact, to study quantum groups, we have to also consider non-commutative $A$-algebras. We denote by $(\mathrm{NCAlg} / A)$ the category of not necessarily commutative $A$-algebras $B$ such that $A$ (or to be more precise, the image of $A$ in $B$ ) is contained in the center of $B$. 
Part I. Quantization of $q$-skew iterative $\sigma$-differential equations

\section{Foundation of a general Galois theory [22, 24, 25]}

\subsection{Notation}

Let us recall basic notation. Let $(R, \delta)$ be a differential ring so that $\delta$ : $R \rightarrow R$ is a derivation of a commutative ring $R$ of characteristic 0 . When there is no danger of confusion we simply say that $R$ is a differential ring, without referring to the derivation $\delta$. We often have to talk, however, about the abstract ring $R$ that we denote by $R^{\natural}$. For a commutative ring $S$ of characteristic 0 , the power series ring $S[[X]]$ with derivation $\mathrm{d} / \mathrm{d} X$ gives us an example of differential ring.

\subsection{General Galois theory of differential field extensions}

Let us start by recalling our general Galois theory of differential field extensions.

\subsubsection{Universal Taylor morphism}

Let $(R, \delta)$ be a differential algebra so that $R$ is a commutative $C$-algebra and $\delta: R \rightarrow R$ is a $C$-derivation:

(1) $\delta: R \rightarrow R$ is a $C$-linear map.

(2) $\delta(a b)=\delta(a) b+a \delta(b)$ for all $a, b \in R$.

For the differential algebra $(R, \delta)$ and a commutative $C$-algebra $S$, a Taylor morphism is a differential morphism

$$
(R, \delta) \longrightarrow(S[[X]], \mathrm{d} / \mathrm{d} X) .
$$

Given a differential ring $(R, \delta)$, among the Taylor morphisms (2.1), there exists the universal one. In fact, for an element $a \in R$, we define the power series

$$
\iota(a)=\sum_{n=0}^{\infty} \frac{1}{n !} \delta^{n}(a) X^{n} \in R^{\natural}[[X]] .
$$

Then the map

$$
\iota:(R, \delta) \longrightarrow\left(R^{\natural}[[X]], \mathrm{d} / \mathrm{d} X\right)
$$

is the universal Taylor morphism. 


\subsubsection{Galois hull $\mathcal{L} / \mathcal{K}$ for a differential field extension $L / k$}

Let $(L, \delta) /(k, \delta)$ be a differential field extension such that the abstract field $L^{\natural}$ is finitely generated over the abstract base field $k^{\natural}$. We constructed the Galois hull $\mathcal{L} / \mathcal{K}$ in the following manner.

We take a mutually commutative basis

$$
\left\{D_{1}, D_{2}, \ldots, D_{d}\right\}
$$

of the $L^{\natural}$-vector space $\operatorname{Der}\left(L^{\natural} / k^{\natural}\right)$ of $k^{\natural}$-derivations of the abstract field $L^{\natural}$. So we have

$$
\left[D_{i}, D_{j}\right]=D_{i} D_{j}-D_{j} D_{i}=0 \quad \text { for } \quad 1 \leqslant i, j \leqslant d .
$$

Now we introduce a partial differential structure on the abstract field $L^{\natural}$ using the derivations $\left\{D_{1}, D_{2}, \ldots, D_{d}\right\}$. Namely we set

$$
L^{\sharp}:=\left(L^{\natural},\left\{D_{1}, D_{2}, \ldots, D_{d}\right\}\right)
$$

that is a partial differential field. Similarly we define a differential structure on the power series ring $L^{\natural}[[X]]$ with coefficients in $L^{\natural}$ by considering the derivations

$$
\left\{D_{1}, D_{2}, \ldots, D_{d}\right\}
$$

that operate on the coefficients of the power series. In other words, we work with the differential ring $L^{\sharp}[[X]]$. So the power series ring $L^{\natural}[[X]]$ has differential structure

$$
\left\{\mathrm{d} / \mathrm{d} X, D_{1}, D_{2}, \ldots, D_{d}\right\}
$$

that consists of the differentiation $\mathrm{d} / \mathrm{d} X$ with respect to the variable $X$ and the derivations given before. Since there is no danger of confusion of the choice of the differential operator $\mathrm{d} / \mathrm{d} X$, we denote this differential ring by

$$
L^{\sharp}[[X]] .
$$

We have the universal Taylor morphism

$$
\iota: L \longrightarrow L^{\natural}[[X]]
$$

that is a differential morphism. We added further the $\left\{D_{1}, D_{2}, \ldots, D_{d}\right\}$ differential structure on $L^{\mathrm{\natural}}[[X]]$ or we replace the target space $L^{\mathrm{\natural}}[[X]]$ of the universal Taylor morphism (2.3) by $L^{\sharp}[[X]]$ so that we have

$$
\iota: L \longrightarrow L^{\sharp}[[X]] \text {. }
$$

In Definition 2.1 below, we work in the differential $\operatorname{ring} L^{\sharp}[[X]]$ with differential operators $\mathrm{d} / \mathrm{d} X$ and $\left\{D_{1}, D_{2}, \ldots, D_{d}\right\}$. We identify the differential field $L^{\sharp}$ with the set of power series consisting only of constant terms. 
Namely,

$$
L^{\sharp}=\left\{\sum_{n=0}^{\infty} a_{n} X^{n} \in L^{\sharp}[[X]] \mid \text { The coefficients } a_{n}=0 \text { for every } n \geqslant 1\right\} .
$$

Therefore $L^{\sharp}$ is a differential sub-field of the differential ring $L^{\sharp}[[X]]$. The differential operator d/d $X$ kills $L^{\sharp}$. Similarly, we set

$$
k^{\sharp}:=\left\{\sum_{n=0}^{\infty} a_{n} X^{n} \in L^{\sharp}[[X]] \mid \begin{array}{l}
\text { The coefficients } a_{0} \in k \\
\text { and } a_{n}=0 \text { for every } n \geqslant 1
\end{array}\right\} .
$$

So all the differential operators $\mathrm{d} / \mathrm{d} X, D_{1}, D_{2}, \ldots, D_{d}$ act trivially on $k^{\sharp}$ and so $k^{\sharp}$ is a differential sub-field of $L^{\sharp}$ and hence of the differential algebra $L^{\sharp}[[X]]$.

Definition 2.1. - The Galois hull $\mathcal{L} / \mathcal{K}$ is the differential sub-algebra of $L^{\sharp}[[X]]$, where $\mathcal{L}$ is the differential sub-algebra generated by the image $\iota(L)$ and $L^{\sharp}$ and $\mathcal{K}$ is the sub-algebra generated by the image $\iota(k)$ and $L^{\sharp}$. So $\mathcal{L} / \mathcal{K}$ is a differential algebra extension with differential operators $\mathrm{d} / \mathrm{d} X$ and $\left\{D_{1}, D_{2}, \ldots, D_{d}\right\}$.

\subsubsection{Universal Taylor morphism for a partial differential ring}

The universal Taylor morphism has a generalization for partial differential ring. Let

$$
\left(R,\left\{\partial_{1}, \partial_{2}, \ldots, \partial_{d}\right\}\right)
$$

be a partial differential ring. So $R$ is a commutative ring of characteristic 0 and $\partial_{i}: R \rightarrow R$ are mutually commutative derivations. For a ring $S$, the power series ring

$$
\left(S\left[\left[X_{1}, X_{2}, \ldots, X_{d}\right]\right],\left\{\frac{\partial}{\partial X_{1}}, \frac{\partial}{\partial X_{2}}, \ldots, \frac{\partial}{\partial X_{d}}\right\}\right)
$$

gives us an example of partial differential ring.

A Taylor morphism is a differential morphism

$$
\begin{aligned}
\left(R,\left\{\partial_{1}, \partial_{2}, \ldots, \partial_{d}\right\}\right) & \\
\longrightarrow & \left(S\left[\left[X_{1}, X_{2}, \ldots, X_{d}\right]\right],\left\{\frac{\partial}{\partial X_{1}}, \frac{\partial}{\partial X_{2}}, \ldots, \frac{\partial}{\partial X_{d}}\right\}\right) .
\end{aligned}
$$

For a differential algebra $\left(R,\left\{\partial_{1}, \partial_{2}, \ldots, \partial_{d}\right\}\right)$, among Taylor morphisms (2.4), there exists the universal one $\iota_{R}$ given below. 
Definition 2.2. - The universal Taylor morphism is a differential morphism

$$
\begin{aligned}
\iota_{R}:\left(R,\left\{\partial_{1}, \partial_{2}, \ldots, \partial_{d}\right\}\right) \\
\longrightarrow\left(R^{\natural}\left[\left[X_{1}, X_{2}, \ldots, X_{d}\right]\right],\left\{\frac{\partial}{\partial X_{1}}, \frac{\partial}{\partial X_{2}}, \ldots, \frac{\partial}{\partial X_{d}}\right\}\right)
\end{aligned}
$$

defined by the formal power series expansion

$$
\iota_{R}(a)=\sum_{n \in \mathbb{N}^{d}} \frac{1}{n !} \partial^{n}(a) X^{n}
$$

for an element $a \in R$, where we use the standard notation for multi-index.

Namely, for $n=\left(n_{1}, n_{2}, \ldots, n_{d}\right) \in \mathbb{N}^{d}$,

$$
|n|=\sum_{i=1}^{d} n_{i}, \quad \partial^{n}=\partial_{1}^{n_{1}} \partial_{2}^{n_{2}} \ldots \partial_{d}^{n_{d}} \quad n !=n_{1} ! n_{2} ! \ldots n_{d} !
$$

and

$$
X^{n}=X_{1}^{n_{1}} X_{2}^{n_{2}} \ldots X_{d}^{n_{d}} .
$$

See [22, Proposition (1.4)].

\subsubsection{The functor $\mathcal{F}_{L / k}$ of infinitesimal deformations for a differ- ential field extension}

For the partial differential field $L^{\sharp}$, we have the universal Taylor morphism

$$
\iota_{L^{\sharp}}: L^{\sharp} \longrightarrow L^{\natural}\left[\left[W_{1}, W_{2}, \ldots, W_{d}\right]\right]=L^{\natural}[[W]],
$$

where we replaced the variables $X$ 's in $(2.5)$ by the variables $W$ 's for a notational reason. The universal Taylor morphism (2.6) gives a differential morphism

$$
L^{\sharp}[[X]] \longrightarrow L^{\natural}\left[\left[W_{1}, W_{2}, \ldots, W_{d}\right]\right][[X]] .
$$

Restricting the morphism (2.7) to the differential sub-algebra $\mathcal{L}$ of $L^{\sharp}[[X]]$, we get a differential morphism $\mathcal{L} \rightarrow L^{\natural}\left[\left[W_{1}, W_{2}, \ldots, W_{d}\right]\right][[X]]$ that we denote by $\iota$. So we have the differential morphism

$$
\iota: \mathcal{L} \longrightarrow L^{\natural}\left[\left[W_{1}, W_{2}, \ldots, W_{d}\right]\right][[X]] .
$$

Similarly for every commutative $L^{\natural}$-algebra $A$, thanks to the differential morphism

$$
\begin{gathered}
L^{\natural}[[W]] \longrightarrow A[[W]] \\
\quad-1327-
\end{gathered}
$$


arising from the structural morphism $L^{\natural} \rightarrow A$, we have the canonical differential morphism

$$
\iota: \mathcal{L} \longrightarrow A\left[\left[W_{1}, W_{2}, \ldots, W_{d}\right]\right][[X]] .
$$

We define the functor

$$
\mathcal{F}_{L / k}:\left(A l g / L^{\natural}\right) \longrightarrow(\text { Set })
$$

from the category ( $\left.\mathrm{Alg} / L^{\natural}\right)$ of commutative $L^{\natural}$-algebras to the category (Set) of sets, by associating to an $L^{\natural}$-algebra $A$, the set of infinitesimal deformations of the canonical morphism (2.8). So

$$
\begin{aligned}
& \mathcal{F}_{L / k}(A) \\
& =\left\{\begin{array}{l|l}
f: \mathcal{L} \rightarrow A\left[\left[W_{1}, W_{2}, \ldots, W_{d}\right]\right][[X]] & \begin{array}{l}
f \text { is a differential morphism congruent } \\
\text { to the canonical morphism } \iota \text { modulo } \\
\text { nilpotent elements such that } f=\iota \\
\text { when restricted on the sub-algebra } \mathcal{K}
\end{array}
\end{array}\right\} .
\end{aligned}
$$

\subsubsection{Group functor Inf-Gal $(L / k)$ of infinitesimal automorphisms for a differential field extension}

The Galois group in our Galois theory is the group functor

$$
\operatorname{Inf-Gal}(L / k):\left(\operatorname{Alg} / L^{\natural}\right) \longrightarrow(\operatorname{Grp})
$$

defined by

$$
\begin{aligned}
& \operatorname{Inf-Gal}(L / k)(A) \\
& =\left\{\begin{array}{l|l}
f: \mathcal{L} \widehat{\otimes}_{L^{\sharp}} A[[W]] \rightarrow \mathcal{L} \widehat{\otimes}_{L^{\sharp}} A[[W]] & \begin{array}{l}
f \text { is a differential } \mathcal{K} \widehat{\otimes}_{L^{\sharp}} A[[W]] \text {-automorphism } \\
\text { continuous with respect to the } W \text {-adic } \\
\text { topology and congruent to the identity } \\
\text { modulo nilpotent elements }
\end{array}
\end{array}\right\}
\end{aligned}
$$

for a commutative $L^{\natural}$-algebra $A$. Here the completion is taken with respect to the $W$-adic topology. See [16, Definition 2.19].

Then the group functor $\operatorname{Inf}-\operatorname{Gal}(L / k)$ operates on the functor $\mathcal{F}_{L / k}$ in such a way that the operation $\left(\operatorname{Inf}-\operatorname{Gal}(L / k), \mathcal{F}_{L / k}\right)$ is a torsor $[22$, Theorem (5.11)].

\subsubsection{Origin of the group structure}

For the differential equations, the Galois group is a group functor. We are going to generalize differential Galois theory in such a way that the Galois group is a quantum group. Quantum group is a generalization of 
affine algebraic group. We can not, however, regard a quantum group as a group functor. Therefore, we have to understand the origin of the group functor Inf-Gal. We illustrate it by an example.

Example 2.3. - Let us consider a differential field extension

$$
L / k:=(\mathbb{C}(y), \delta) / \mathbb{C}
$$

such that $y$ is transcendental over the field $\mathbb{C}$ and

$$
\delta(y)=y \quad \text { and } \quad \delta(\mathbb{C})=0 .
$$

Thus $k=\mathbb{C}$ is the field of constants of $L$.

The universal Taylor morphism

$$
\iota: L \longrightarrow L^{\natural}[[X]]
$$

maps $y \in L$ to

$$
Y:=y \exp X \in L^{\natural}[[X]] .
$$

Since the $L^{\natural}$-vector space $\operatorname{Der}\left(L^{\natural} / k^{\natural}\right)$ is one-dimensional with basis d/dy, we have $L^{\sharp}=\left(L^{\natural}, \mathrm{d} / \mathrm{d} y\right)$. Since we have the relations

$$
\frac{\partial Y}{\partial X}=Y, \quad y \frac{\partial Y}{\partial y}=Y
$$

in the power series ring $L^{\sharp}[[X]]$, the Galois hull $\mathcal{L} / \mathcal{K}$ is given by

$$
\mathcal{L}=\mathcal{K} . \mathbb{C}(\exp X), \quad \mathcal{K}=L^{\sharp} \subset L^{\sharp}[[X]] .
$$

Now let us look at the infinitesimal deformation functor $\mathcal{F}_{L / k}$. To this end, we Taylor-expand the coefficients of the power series in $L^{\sharp}[[X]]$ to get

$$
\iota: L \longrightarrow L^{\sharp}[[X]] \longrightarrow L^{\natural}[[W]][[X]]=L^{\natural}[[W, X]]
$$

so that

$$
\iota(y)=(y+W) \exp X \in L^{\natural}[[W, X]] .
$$

We identify $L^{\sharp}[[X]]$ with its image in $L^{\natural}[[W]][[X]]=L^{\natural}[[W, X]]$. In particular we identify $Y=y \exp X \in L^{\sharp}[[X]]$ with $Y(W, X)=(y+W) \exp X \in$ $L^{\natural}[[W, X]]$. Equalities $(2.11)$ become in $L^{\natural}[[W, X]]$

$$
\frac{\partial Y(W, X)}{\partial X}=Y(W, X), \quad(y+W) \frac{\partial Y(W, X)}{\partial W}=Y
$$

It follows from (2.13) that for a commutative $L^{\natural}$-algebra $A$, an infinitesimal deformation $\varphi \in \mathcal{F}_{L / k}(A)$ is determined by the image

$$
\varphi(Y(W, X))=c Y(W, X) \in A[[W, X]],
$$

where $c \in A$. Conversely any invertible element $c \in A$ infinitesimally close to 1 defines an infinitesimal deformation so that we conclude

$$
\mathcal{F}_{L / k}(A)=\{c \in A \mid c-1 \text { is nilpotent }\} .
$$


Where does the group structure come from?

There are two ways of answering to this question, which are closely related.

(I) Algebraic answer. - By (2.14), we have

$$
\varphi(y)=c(y+W) \exp X \in A[[W, X]],
$$

where $c-1 \in A$ is a nilpotent element. Consequently we have

$$
\varphi(y)=Y((c-1) y+c W, X) .
$$

In other words, $\varphi(y)$ coincides with

$$
\left.Y(W, X)\right|_{W=(c-1) y+c W} .
$$

Equivalently $\varphi(y)$ is obtained by substituting $(c-1) y+c W$ for $W$ in $Y(W, X)$. This is quite natural in view of differential equations (2.13). We have only to look at the initial condition at $X=0$ of the two solutions

$$
Y(W, X), \quad \varphi(y)=c(y+W) Y(W, X)
$$

of the differential equation $\partial \mathrm{Y} / \partial X=\mathrm{Y}$. The transformation

$$
W \longmapsto(c-1) y+c W \text { where } c \in A \text { and } c-1 \text { is nilpotent, }
$$

is an infinitesimal coordinate transformation of the initial condition and the multiplicative structure of $c$ is nothing but the composite of coordinate transformations (2.17).

(II) Geometric answer. - For this let us look at the dynamical system defined by the differential equation (2.10); it is the dynamical system on the line $\mathbb{C}$ described by

$$
y \mapsto Y=y \exp X .
$$

Observing the dynamical system through algebraic differential equations is equivalent to considering the deformations of the Galois hull. So the (infinitesimal) deformation functor measures the ambiguity of the observation. In other words, the result due to our method is (2.15). In terms of the initial condition, it looks as

$$
\left.y \mapsto c Y\right|_{X=0}=\left.c y \exp X\right|_{X=0}=c y .
$$

Namely,

$$
y \mapsto c y .
$$

If we have two transformations (2.18)

$$
y \mapsto c y, \quad y \mapsto c^{\prime} y
$$

the composite transformation corresponds to the product

$$
y \mapsto c c^{\prime} y .
$$

Our generalization depends on the first answer (I). See Section 7. 


\subsection{Difference Galois theory}

If we replace the universal Taylor morphism by the universal Euler morphism, we can construct a general Galois theory of difference equations $[16,17]$.

\subsubsection{Universal Euler morphism}

Let $(R, \sigma)$ be a $C$-difference algebra so that $\sigma: R \rightarrow R$ is a $C$-algebra automorphism of a commutative $C$-algebra $R$. See Remark 3.7. When there is no danger of confusion we simply say that $R$ is a difference ring, without referring to the automorphism $\sigma$. We often have to talk however about the abstract ring $R$ that we denote by $R^{\natural}$. For a commutative $\operatorname{ring} S$, we denote by $F(\mathbb{Z}, S)$ the ring of functions on the set of integers $\mathbb{Z}$ taking values in the ring $S$. For a function $f \in F(\mathbb{Z}, S)$, we define the shifted function $\Sigma f \in$ $F(\mathbb{Z}, S)$ by

$$
(\Sigma f)(n)=f(n+1) \quad \text { for every } n \in \mathbb{Z} .
$$

Hence the shift operator

$$
\Sigma: F(\mathbb{Z}, S) \longrightarrow F(\mathbb{Z}, S)
$$

is an automorphism of the $\operatorname{ring} F(\mathbb{Z}, S)$ so that $(F(\mathbb{Z}, S), \Sigma)$ is a difference ring.

Remark 2.4. - In this Paragraph 2.3.1 and the next 2.3.2, in particular for the existence of the universal Euler morphism, we do not need the commutativity assumption of the underlying ring.

Let $(R, \sigma)$ be a difference ring and $S$ a ring. An Euler morphism is a difference morphism

$$
(R, \sigma) \longrightarrow(F(\mathbb{Z}, S), \Sigma) .
$$

Given a difference ring $(R, \sigma)$, among the Euler morphisms (2.19), there exists the universal one. In fact, for an element $a \in R$, we define the function $u[a] \in F\left(\mathbb{Z}, R^{\natural}\right)$ by

$$
u[a](n)=\sigma^{n}(a) \quad \text { for } n \in \mathbb{Z} .
$$

Then the map

$$
\iota:(R, \sigma) \longrightarrow\left(F\left(\mathbb{Z}, R^{\natural}\right), \Sigma\right) \quad a \longmapsto u[a]
$$

is the universal Euler morphism [16, Proposition 2.5]. 


\subsubsection{Galois hull $\mathcal{L} / \mathcal{K}$ for a difference field extension $L / k$}

Let $(L, \sigma) /(k, \sigma)$ be a difference field extension such that the abstract field $L^{\natural}$ is finitely generated over the abstract base field $k^{\natural}$. We constructed the Galois hull $\mathcal{L} / \mathcal{K}$ as in the differential case. Namely, we take a mutually commutative basis

$$
\left\{D_{1}, D_{2}, \ldots, D_{d}\right\}
$$

of the $L^{\natural}$-vector space $\operatorname{Der}\left(L^{\natural} / k^{\natural}\right)$ of $k^{\natural}$-derivations of the abstract field $L^{\natural}$.

We introduce the partial differential field

$$
L^{\sharp}:=\left(L^{\natural},\left\{D_{1}, D_{2}, \ldots, D_{d}\right\}\right) .
$$

Similarly we define a differential structure on the $\operatorname{ring} F\left(\mathbb{Z}, L^{\natural}\right)$ of functions taking values in $L^{\natural}$ by considering the derivations

$$
\left\{D_{1}, D_{2}, \ldots, D_{d}\right\} \text {. }
$$

In other words, we work with the differential ring $F\left(\mathbb{Z}, L^{\sharp}\right)$. So the ring $F\left(\mathbb{Z}, L^{\natural}\right)$ has the difference-differential structure

$$
\left\{\Sigma, D_{1}, \ldots, D_{d}\right\}
$$

that consists of the shift operator $\Sigma$ and the derivations given before. Since there is no danger of confusion of the choice of the difference operator $\Sigma$, we denote this difference-differential ring by

$$
F\left(\mathbb{Z}, L^{\sharp}\right) .
$$

We have the universal Euler morphism

$$
\iota: L \longrightarrow F\left(\mathbb{Z}, L^{\natural}\right)
$$

that is a difference morphism. We added further the $\left\{D_{1}, D_{2}, \ldots, D_{d}\right\}$-differential structure on $F\left(\mathbb{Z}, L^{\natural}\right)$ or we replace the target space $F\left(\mathbb{Z}, L^{\natural}\right)$ of the universal Euler morphism $(2.21)$ by $F\left(\mathbb{Z}, L^{\sharp}\right)$ so that we have

$$
\iota: L \longrightarrow F\left(\mathbb{Z}, L^{\sharp}\right) \text {. }
$$

In Definition 2.5 below, we work in the difference-differential $\operatorname{ring} F\left(\mathbb{Z}, L^{\sharp}\right)$ with difference operator $\Sigma$ and differential operators $\left\{D_{1}, D_{2}, \ldots, D_{d}\right\}$. We identify with $L^{\sharp}$ the set of constant functions on $\mathbb{Z}$. Namely,

$$
L^{\sharp}=\left\{f \in F\left(\mathbb{Z}, L^{\sharp}\right) \mid f(0)=f( \pm 1)=f( \pm 2)=\cdots \in L^{\sharp}\right\} .
$$

Therefore $L^{\sharp}$ is a difference-differential sub-field of the difference-differential $\operatorname{ring} F\left(\mathbb{Z}, L^{\sharp}\right)$. The action of the shift operator on $L^{\sharp}$ being trivial, the notation is adequate. Similarly, we set

$$
k^{\sharp}:=\left\{f \in F\left(\mathbb{Z}, L^{\sharp}\right) \mid f(0)=f( \pm 1)=f( \pm 2)=\cdots \in k \subset L^{\sharp}\right\} .
$$


So both the shift operator and the derivations act trivially on $k^{\sharp}$ and so $k^{\sharp}$ is a difference-differential sub-field of $L^{\sharp}$ and hence of the difference-differential algebra $F\left(\mathbb{Z}, L^{\sharp}\right)$.

Definition 2.5. - The Galois hull $\mathcal{L} / \mathcal{K}$ is a difference-differential subalgebra extension of $F\left(\mathbb{Z}, L^{\sharp}\right)$, where $\mathcal{L}$ is the difference-differential subalgebra generated by the image $\iota(L)$ and $L^{\sharp}$ and $\mathcal{K}$ is the sub-algebra generated by the image $\iota(k)$ and $L^{\sharp}$. So $\mathcal{L} / \mathcal{K}$ is a difference-differential algebra extension with difference operator $\Sigma$ and derivations $\left\{D_{1}, D_{2}, \ldots, D_{d}\right\}$.

\subsubsection{The functor $\mathcal{F}_{L / k}$ of infinitesimal deformations for a differ- ence field extension}

For the partial differential field $L^{\sharp}$, we have the universal Taylor morphism

$$
\iota_{L^{\sharp}}: L^{\sharp} \longrightarrow L^{\natural}\left[\left[W_{1}, W_{2}, \ldots, W_{d}\right]\right]=L^{\natural}[[W]] .
$$

The universal Taylor morphism (2.22) gives a difference-differential morphism

$$
F\left(\mathbb{Z}, L^{\sharp}\right) \longrightarrow F\left(\mathbb{Z}, L^{\natural}\left[\left[W_{1}, W_{2}, \ldots, W_{d}\right]\right]\right) .
$$

Restricting the morphism (2.23) to the difference-differential sub-algebra $\mathcal{L}$ of $F\left(\mathbb{Z}, L^{\sharp}\right)$, we get a difference-differential morphism $\mathcal{L} \rightarrow F\left(\mathbb{Z}, L^{\natural}\left[\left[W_{1}\right.\right.\right.$, $\left.\left.\left.W_{2}, \ldots, W_{d}\right]\right]\right)$ that we denote by $\iota$. So we have the difference-differential morphism

$$
\iota: \mathcal{L} \longrightarrow F\left(\mathbb{Z}, L^{\natural}\left[\left[W_{1}, W_{2}, \ldots, W_{d}\right]\right]\right) .
$$

For every commutative $L^{\natural}$-algebra $A$, the structure morphism $L^{\natural} \rightarrow A$ gives rise to the differential morphism

$$
L^{\natural}[[W]] \longrightarrow A[[W]],
$$

and it induces the canonical difference-differential morphism

$$
\iota: \mathcal{L} \longrightarrow F\left(\mathbb{Z}, A\left[\left[W_{1}, \ldots, W_{d}\right]\right]\right)
$$

which is analogous to (and generalizes, in fact) the one in (2.24).

We define the functor

$$
\mathcal{F}_{L / k}:\left(\mathrm{Alg} / L^{\natural}\right) \longrightarrow(\mathrm{Set})
$$

from the category (Alg $/ L^{\natural}$ ) of commutative $L^{\natural}$-algebras to the category (Set) of sets, by associating to a commutative $L^{\natural}$-algebra $A$, the set of infinitesimal 
deformations of the canonical morphism (2.24). So

$\mathcal{F}_{L / k}(A)$

$=\left\{\begin{array}{l|l}f: \mathcal{L} \rightarrow F\left(\mathbb{Z}, A\left[\left[W_{1}, W_{2}, \ldots, W_{d}\right]\right]\right) & \begin{array}{l}f \text { is a difference-differential morphism } \\ \text { congruent to the canonical morphism } \iota \\ \text { modulo nilpotent elements such that } \\ f=\iota \text { when restricted on the sub-algebra } \mathcal{K}\end{array}\end{array}\right\}$.

See [16, Definition 2.13] for a rigorous definition.

\subsubsection{Group functor Inf-Gal $(L / k)$ of infinitesimal automorphisms for a difference field extension}

The Galois group in our Galois theory is the group functor

$$
\operatorname{Inf}-\operatorname{Gal}(L / k):\left(\operatorname{Alg} / L^{\natural}\right) \longrightarrow(\operatorname{Grp})
$$

defined by

$$
\begin{aligned}
& \operatorname{Inf-Gal}(L / k)(A)
\end{aligned}
$$

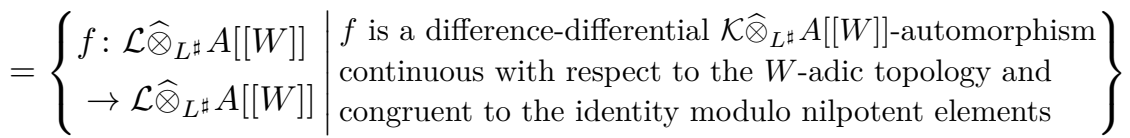

for a commutative $L^{\natural}$-algebra $A$. Here the completion is taken with respect to the $W$-adic topology. See [16, Definition 2.19].

Then the group functor $\operatorname{Inf}-\operatorname{Gal}(L / k)$ operates on the functor $\mathcal{F}_{L / k}$ in such a way that the operation $\left(\operatorname{Inf}-\operatorname{Gal}(L / k), \mathcal{F}_{L / k}\right)$ is a torsor [16, Theorem 2.20].

The group functor $\operatorname{Inf}-\operatorname{Gal}(L / k)$ arises from the same origin as in the differential case, namely from automorphisms of the initial conditions as we explained in 2.2.6. The principle is the same in the quantum case as well, in which case the Galois hulls will turn, however, to be non-commutative. We are going to show by examples that the principle works well to produce quantum groups as Galois groups. The examples are three, given in Sections 4-6 below, each.

\subsection{Introduction of more precise notations}

So far, we explained general differential Galois theory and general difference Galois theory. To go further, we have to make our notations more precise. 
For example, we defined the Galois hull for a differential field extension in Definition 2.1 and the Galois hull for a difference field extension in Definition 2.5. Since they are defined by the same principle, we denoted both of them by $\mathcal{L} / \mathcal{K}$. We have to, however, distinguish them.

Definition 2.6. - We denote the Galois hull for a differential field extension by $\mathcal{L}_{\delta} / \mathcal{K}_{\delta}$ and we use the symbol $\mathcal{L}_{\sigma} / \mathcal{K}_{\sigma}$ for the Galois hull of a difference field extension.

We also have to distinguish the functors $\mathcal{F}_{L / k}$ and $\operatorname{Inf}-\mathrm{Gal}(L / k)$ in the differential case and in the difference case: we add the suffix $\delta$ for the differential case and the suffix $\sigma$ for the difference case:

(1) We use $\mathcal{F}_{\delta L / k}$ and $\operatorname{Inf}_{-G^{\prime}}(L / k)$, when we deal with differential algebras.

(2) We use $\mathcal{F}_{\sigma L / k}$ and $\operatorname{Inf}_{-G^{\prime}}(L / k)$ for difference algebras.

\section{Hopf Galois theory}

Picard-Vessiot theory is a Galois theory of linear differential or difference equations. The idea of introducing Hopf algebra in Picard-Vessiot theory is traced back to Sweedler [20]. Specialists in Hopf algebra succeeded in unifying Picard-Vessiot theories for differential equations and difference equations [1]. They further succeeded in generalizing the Picard-Vessiot theory for difference-differential equations, where the operators are not necessarily commutative. Heiderich [7] combined the idea of Picard-Vessiot theory via Hopf algebra with our general Galois theory for non-linear equations [16, 22]. This is a wonderful idea. By our examples it will become, however, apparent that his results require a certain modification in the non-co-commutative case. His general theory includes a wide class of difference and differential algebras.

\subsection{The idea of Heiderich's theory}

There are two major advantages in Heiderich's theory.

(1) Unified study of differential equations and difference equations in non-linear case.

(2) Generalization of universal Euler morphism and Taylor morphism. 
For vector spaces $M, N$ over the field $C$, we denote by ${ }_{C} \mathbf{M}(M, N)$ the $C$-vector space of $C$-linear maps from $M$ to $N$. Let $\mathcal{H}$ be a $C$-Hopf algebra or more generally, a $C$-bi-algebra, and let $A$ be a commutative $C$-algebra. Suppose

$$
\Psi \in_{C} \mathbf{M}\left(A \otimes_{C} \mathcal{H}, A\right)={ }_{C} \mathbf{M}\left(A,_{C} \mathbf{M}(\mathcal{H}, A)\right) .
$$

Thus, $\Psi$ defines two $C$-linear maps

(1) $\Psi_{1}: A \otimes_{C} \mathcal{H} \rightarrow A$,

(2) $\Psi_{2}: A \rightarrow{ }_{C} \mathbf{M}(\mathcal{H}, A)$.

Definition 3.1. - We say that $(A, \Psi)$ is an $\mathcal{H}$-module algebra if the following equivalent conditions (see [20, p. 153]) are satisfied.

(1) The $C$-linear map $\Psi_{1}: A \otimes_{C} \mathcal{H} \rightarrow A$ makes $A$ into a left $\mathcal{H}$-module in such a way that we have in the algebra $A$,

$$
h(a b)=\sum\left(h_{(1)} a\right)\left(h_{(2)} b\right) \in A,
$$

for every element $h \in \mathcal{H}$ and $a, b \in A$, where we use the sigma notation so that

$$
\Delta(h)=\sum h_{(1)} \otimes h_{(2)},
$$

$\Delta: \mathcal{H} \rightarrow \mathcal{H} \otimes \mathcal{H}$ being the co-multiplication of the Hopf algebra $\mathcal{H}$.

(2) The C-linear map

$$
\Psi_{2}: A \longrightarrow{ }_{C} \mathbf{M}(\mathcal{H}, A)
$$

is a $C$-algebra morphism, where ${ }_{C} \mathbf{M}(\mathcal{H}, A)$ forms a $C$-algebra with respect to the convolution product [20, p. 70].

Example 3.2. - To recognize Heiderich's idea for the example of differential algebra, suppose that $\mathcal{H}$ is the $C$-Hopf algebra $C\left[\mathbb{G}_{a C}\right]=C[t]$ of the coordinate ring of the additive group scheme $\mathbb{G}_{a C}$ over the field $C$. Then the dual algebra ${ }_{C} \mathbf{M}(\mathcal{H}, A)$ is the formal power series ring $A[[X]]$. It is a comfortable exercise to examine that $(A, \Psi)$ is an $\mathcal{H}$-module algebra if and only if $A$ is a differential algebra with derivation $\delta$ such that $\delta(C)=0$, when we define $\delta$ by $\Psi(a \otimes t)=\delta(a)$ for every $a \in A$. Notice that the $C$-algebra morphism

$$
\Psi_{2}: A \longrightarrow{ }_{C} \mathbf{M}(\mathcal{H}, A)=A[[X]]
$$

is precisely the universal Taylor morphism. Indeed, one sees

$$
\Psi_{2}(a)=\sum_{n=0}^{\infty} \frac{1}{n !} \delta^{n}(a) X^{n} \in A[[X]]
$$

for every $a \in A$. See Heiderich [7, 2.3.4]. 
If we alternatively take as $\mathcal{H}$, the Hopf algebra $C\left[\mathbb{G}_{m C}\right]$ of the coordinate ring of the multiplicative group $\mathbb{G}_{m C}$ for $\mathcal{H}$, then we get difference structure and the universal Euler morphism. See [7, 2.3.1]. Indeed, Heiderich takes as $\mathcal{H}$ any co-commutative Hopf algebra to get an algebra $A$ with operation of the algebra $\mathcal{H}$ and a morphism

$$
\Psi_{2}: A \longrightarrow{ }_{C} \mathbf{M}(\mathcal{H}, A)
$$

generalizing the universal Taylor morphism and Euler morphism. He then defined the Galois hull $\mathcal{L} / \mathcal{K}$ and developed a general Galois theory for a field extension $L / k$ with operation of the algebra $\mathcal{H}$. As a point the cocommutativity assumption on $\mathcal{H}$ makes the dual algebra ${ }_{C} \mathrm{M}(\mathcal{H}, A)$ into a commutative algebra, and the Galois hull $\mathcal{L} / \mathcal{K}$ is realized as sub-algebras in the commutative algebra ${ }_{C} \mathbf{M}(\mathcal{H}, A)$. We remark that the Galois hull may be regarded as an algebraic counterpart of the geometric object, algebraic Lie groupoid. See Malgrange [11]. Therefore, the most fascinating question is

QUESTION 3.3. - Let us consider a non-co-commutative bi-algebra $\mathcal{H}$ and assume that the Galois hull $\mathcal{L} / \mathcal{K}$ that is a sub-algebra of the dual algebra ${ }_{C} \mathrm{M}(\mathcal{H}, A)$, is not a commutative algebra. Does the Galois hull $\mathcal{L} / \mathcal{K}$ quantize the algebraic Lie groupoid?

We are going to answer affirmatively the question by analyzing examples of $q$-skew iterative $\sigma$-differential field extensions.

Remark 3.4. - In view of Hardouin [5] and Masuoka and Yanagawa [14], one may think that even if the Hopf algebra $\mathcal{H}$ is non-co-commutative, the Galois hull $\mathcal{L}$ would be a commutative sub-algebra of the non-commutative algebra ${ }_{C} \mathbf{M}(\mathcal{H}, A)$ and the Galois group would be a linear algebraic group, so far as one works only with linear difference-differential equations; see also [26]. However, our examples will show that quantization of Galois theory does occur not only for non-linear equation but also for linear ones; First Example of ours in Section 4 is indeed the first example showing such an unexpected phenomenon.

\section{2. $q$-skew iterative $\sigma$-differential algebra $[5,6]$}

The first non-trivial example of a Hopf Galois theory dependent on a non-co-commutative Hopf algebra is Galois theory of $q$-skew iterative $\sigma$ differential field extensions; they are abbreviated as $q$-SI $\sigma$-differential field extensions. Furthermore we simply call them qsi field extensions. See Definition 3.5. 


\subsubsection{Definition of $q s i$ algebra}

Let $q \neq 0$ be an element of the field $C$. We use a standard notation of $q$-binomial coefficients. To this end, let $Q$ be a variable over the field $C$.

We set $[n]_{Q}=\sum_{i=0}^{n-1} Q^{i} \in C[Q]$ for positive integer $n$. We need also $q$-factorial

$$
[n]_{Q} !:=\prod_{i=1}^{n}[i]_{Q} \text { for a positive integer } n \text { and }[0]_{Q} !:=1 .
$$

So $[n]_{Q} \in C[Q]$. The $Q$-binomial coefficient is defined for $m, n \in \mathbb{N}$ by

$$
\left(\begin{array}{c}
m \\
n
\end{array}\right)_{Q}= \begin{cases}\frac{[m]_{Q} !}{[m-n]_{Q} ![n]_{Q} !} & \text { if } m \geqslant n, \\
0 & \text { if } m<n .\end{cases}
$$

Then we can show that the rational function

$$
\left(\begin{array}{c}
m \\
n
\end{array}\right)_{Q} \in C(Q)
$$

is in fact a polynomial or

$$
\left(\begin{array}{l}
m \\
n
\end{array}\right)_{Q} \in C[Q]
$$

We have a ring morphism

$$
C[Q] \longrightarrow C, \quad Q \longmapsto q
$$

over $C$ and we denote the image of the polynomial

$$
\left(\begin{array}{c}
m \\
n
\end{array}\right)_{Q}
$$

under morphism (3.1) by

$$
\left(\begin{array}{c}
m \\
n
\end{array}\right)_{q}
$$

Definition 3.5. - A q-skew iterative $\sigma$-differential algebra $(A, \sigma$, $\left.\sigma^{-1}, \theta^{*}\right)=\left(A, \sigma,\left\{\theta^{(i)}\right\}_{i \in \mathbb{N}}\right)$, a qsi algebra or a qsi algebra for short, consists of a $C$-algebra $A$ that is eventually non-commutative, a $C$-automorphism $\sigma: A \rightarrow A$ of the $C$-algebra $A$ and a family

$$
\theta^{(i)}: A \longrightarrow A \text { for } i \in \mathbb{N}
$$

of C-linear maps, called derivations, satisfying the following conditions.

(1) $\theta^{(0)}=\operatorname{Id}_{A}$,

(2) $\theta^{(i)} \sigma=q^{i} \sigma \theta^{(i)}$ for every $i \in \mathbb{N}$,

(3) $\theta^{(i)}(a b)=\sum_{l+m=i, l, m \geqslant 0} \sigma^{m}\left(\theta^{(l)}(a)\right) \theta^{(m)}(b)$ for every $i \in \mathbb{N}$ and $a, b \in A$, 
(4) $\theta^{(i)} \circ \theta^{(j)}=\left(\begin{array}{c}i+j \\ i\end{array}\right)_{q} \theta^{(i+j)}$ for every $i, j \in \mathbb{N}$.

We say that an element a of the qsi algebra $A$ is a constant if $\sigma(a)=a$ and $\theta^{(i)}(a)=0$ for every $i \geqslant 1$.

A morphism of qsi $C$-algebras is a $C$-algebra morphism compatible with the automorphisms $\sigma$ and the derivations $\theta^{*}$.

Both differential algebras and difference algebras are qsi algebras as we see below.

Remark 3.6. - There is also a weaker version of qsi algebra, for which we only require $\sigma$ to be an endomorphism, not necessarily an automorphism.

\subsubsection{Difference algebra and a $q s i$ algebra}

Let $A$ be a commutative $C$-algebra and $\sigma: A \rightarrow A$ be a $C$-automorphism of the $\operatorname{ring} A$. So $(A, \sigma)$ is a difference algebra. If we set $\theta^{(0)}=\operatorname{Id}_{A}$ and

$$
\theta^{(i)}(a)=0 \text { for every element } a \in A \text { and for } i=1,2,3, \ldots
$$

Then $\left(A, \sigma, \sigma^{-1}, \theta^{*}\right)$ is a $q s i$ algebra.

Namely we have a functor from the category of $C$-difference algebras to the category of $q s i$ algebras over $C$ :

(Category of Difference Algebras $/ C) \longrightarrow($ Category of qsi Algebras $/ C$ ).

Let $t$ be a variable over the field $C$ and let us now assume

$$
q^{n} \neq 1 \quad \text { for every positive integer } n \text {. }
$$

We denote by $\sigma: C(t) \rightarrow C(t)$ the $C$-automorphism of the rational function field $C(t)$ sending the variable $t$ to $q t$. We consider a difference algebra extension $(A, \sigma) /(C(t), \sigma)$. If we set

$$
\theta^{(1)}(a)=\frac{\sigma(a)-a}{(q-1) t} \quad \text { for every element } a \in A
$$

and

$$
\theta^{(i)}=\frac{1}{[i]_{q} !}\left\{\theta^{(1)}\right\}^{i} \quad \text { for } i=2,3, \ldots
$$

Then $\left(A, \sigma, \sigma^{-1}, \theta^{*}\right)$ is a $q$ si algebra. Therefore if $q \in C$ satisfies $(3.2)$, then we have a functor

(Category of Difference Algebras/ $(C(t), \sigma)$ )

$\longrightarrow$ ( Category of qsi Algebras $\left./\left(C(t), \sigma, \sigma^{-1}, \theta^{*}\right)\right)$. 
Remark 3.7. - In coherence with Remark 3.6, when we speak of difference $C$-algebra $(A, \sigma)$, we assume that $\sigma: A \rightarrow A$ is a $C$-linear automorphism.

\subsubsection{Differential algebra and $q s i$ algebra}

Let $(A, \theta)$ be a $C$-differential algebra such that the derivation $\theta: A \rightarrow A$ is $C$-linear. We set

$$
\theta^{(0)}=\operatorname{Id}_{A}, \quad \theta^{(i)}=\frac{1}{i !} \theta^{i} \quad \text { for } i=1,2,3, \ldots .
$$

Then $\left(A, \operatorname{Id}_{A}, \theta^{*}\right)$ is a $q s i$ algebra for $q=1$. In other words, we have a functor

(Category of Difference Algebras $/ C) \longrightarrow($ Category of qsi Algebras/C)

of the category of (commutative) differential $C$-algebras to the category of $q s i$ algebras over $C$. We have shown that both difference algebras and differential algebras are particular instances of qsi algebra.

\subsubsection{Example of $q s i$ algebra $[7]$}

We are going to see that qsi algebras live on the border between commutative algebras and non-commutative algebras. The examples below would suggest that it is natural to seek qsi algebras in the category of non-commutative algebras.

An example of $q s i$ algebra arises from a commutative $C$-difference algebra $(S, \sigma)$. We need, however, a non-commutative ring, the twisted power series ring $(S, \sigma)[[X]]$ over the difference ring $(S, \sigma)$ that has a natural qsi algebra structure.

Namely, let $(S, \sigma)$ be the $C$-difference ring so that $\sigma: S \rightarrow S$ is a $C$ algebra automorphism of the commutative ring $S$. We introduce the following twisted formal power series ring $(S, \sigma)[[X]]$ with coefficients in $S$ that is the formal power series ring $S[[X]]$ as an additive group with the following commutation relation

$$
a X=X \sigma(a) \quad \text { and } \quad X a=\sigma^{-1}(a) X \quad \text { for every } a \in S .
$$

So more generally

$$
a X^{n}=X^{n} \sigma^{n}(a) \quad \text { and } \quad X^{n} a=\sigma^{-n}(a) X^{n}
$$

for every $n \in \mathbb{N}$. The multiplication of two formal power series is defined by extending (3.4) by linearity. Therefore the twisted formal power series ring 
$(S, \sigma)[[X]]$ is non-commutative in general. By commutation relation (3.4), we can identify

$$
(S, \sigma)[[X]]=\left\{\sum_{i=0}^{\infty} X^{i} a_{i} \mid a_{i} \in S \text { for every } i \in \mathbb{N}\right\}
$$

as additive groups.

We are going to see that the twisted formal power series ring has a natural qsi structure. We define first a ring automorphism

$$
\widehat{\Sigma}:(S, \sigma)[[X]] \longrightarrow(S, \sigma)[[X]]
$$

by setting

$$
\widehat{\Sigma}\left(\sum_{i=0}^{\infty} X^{i} a_{i}\right)=\sum_{i=0}^{\infty} X^{i} q^{i} \sigma\left(a_{i}\right) \quad \text { for every } i \in \mathbb{N},
$$

for every element

$$
\sum_{i=0}^{\infty} X^{i} a_{i} \in(S, \sigma)[[X]] .
$$

As we assume that $\sigma: A \rightarrow A$ is an isomorphism, the $C$-linear map,

$$
\widehat{\Sigma}:(A, \sigma)[[X]] \longrightarrow(A, \sigma)[[X]]
$$

is an automorphism of the $C$-linear space. The operators $\Theta^{*}=\left\{\Theta^{(l)}\right\}_{l \in \mathbb{N}}$ are defined by

$$
\Theta^{(l)}\left(\sum_{i=0}^{\infty} X^{i} a_{i}\right)=\sum_{i=0}^{\infty} X^{i}\left(\begin{array}{c}
i+l \\
l
\end{array}\right)_{q} a_{i+l} \quad \text { for every } l \in \mathbb{N} .
$$

Hence the twisted formal power series $\operatorname{ring}\left((S, \sigma)[[X]], \widehat{\Sigma}, \Theta^{*}\right)$ is a noncommutative qsi ring. We denote this qsi ring simply by $(S, \sigma)[[X]]$. See $[7,2.3]$.

In particular, if we take as the coefficient difference ring $S$ the difference ring

$$
(F(\mathbb{Z}, A), \Sigma)
$$

of functions on $\mathbb{Z}$ taking values in a ring $A$ defined in 2.3.1, where

$$
\Sigma: F(\mathbb{Z}, A) \longrightarrow F(\mathbb{Z}, A)
$$

is the shift operator, we obtain the qsi ring

$$
\left((F(\mathbb{Z}, A), \Sigma)[[X]], \widehat{\Sigma}, \Theta^{*}\right) .
$$

This qsi ring will be denoted simply by $(F(\mathbb{Z}, A), \Sigma)[[X]]$, or even by $F(\mathbb{Z}, A)[[X]]$. 
Remark 3.8. - We assumed that the coefficient difference ring $(S, \sigma)$ is commutative. The commutativity assumption on the ring $S$ is not necessary. Therefore, we may suppose that the ring $A$ in (3.7) is non-commutative.

\subsubsection{Hopf algebra for $q s i$ structures}

As we explained for differential algebras in Definition 3.1, a qsi structure is nothing but an $\mathcal{H}_{q}$-module algebra structure for a Hopf algebra $\mathcal{H}_{q}$.

Definition 3.9. - Let $q \neq 0$ be an element of the field $C$. Let $\mathcal{H}_{q}$ be the $C$-algebra which is generated by $s, s^{-1}$ and $t_{i}, i \in \mathbb{N}$, and is defined by the relations

$$
\begin{gathered}
t_{0}=1, \quad s s^{-1}=s^{-1} s=1, \quad t_{i} s=q^{i} s t_{i}, \\
q^{i} t_{i} s^{-1}=s^{-1} t_{i}, \quad t_{i} t_{j}=\left(\begin{array}{c}
i+j \\
i
\end{array}\right)_{q} t_{i+j}
\end{gathered}
$$

for every $i, j \in \mathbb{N}$. We define a co-algebra structure $\Delta: \mathcal{H}_{q} \rightarrow \mathcal{H}_{q} \otimes_{C} \mathcal{H}_{q}$ by

$$
\Delta(s)=s \otimes s, \quad \Delta\left(s^{-1}\right)=s^{-1} \otimes s^{-1}, \quad \Delta\left(t_{l}\right)=\sum_{i=0}^{l} s^{i} t_{l-i} \otimes t_{i}
$$

for every $l \in \mathbb{N}$. In fact $\mathcal{H}_{q}$ is a Hopf algebra with co-unit $\epsilon: \mathcal{H}_{q} \rightarrow C$ defined by

$$
\epsilon(s)=\epsilon\left(s^{-1}\right)=1, \quad \epsilon\left(t_{i}\right)=0
$$

for every $i \in \mathbb{N}$. Antipode is an anti-automorphism $S: \mathcal{H}_{q} \rightarrow \mathcal{H}_{q}$ of the C-algebra $\mathcal{H}_{q}$ given by

$$
S(s)=s^{-1}, \quad S\left(s^{-1}\right)=s, \quad S\left(t_{i}\right)=(-1)^{i} q^{i(i+1) / 2} t_{i} s^{-i}
$$

for every $i \in \mathbb{N}$.

Proposition 3.10. - For a not necessarily commutative $C$-algebra $A$, there exists a 1:1 correspondence between the elements of the following two sets.

(1) The set of qsi algebra structures on the $C$-algebra $A$.

(2) The set of $\mathcal{H}_{q}$-module algebra structures on the $C$-algebra $A$.

This result is well-known. See Heiderich [7]. We recall for a qsi algebra $A$, the corresponding left $\mathcal{H}_{q}$-module structure is given by

$$
s \longmapsto \sigma, \quad s^{-1} \longmapsto \sigma^{-1}, \quad t_{i} \longmapsto \theta^{(i)} \quad \text { for every } i \in \mathbb{N} \text {. }
$$




\subsubsection{Universal Hopf morphism for a qsi algebra}

We introduced in 2.3.1 the difference ring of functions $(F(\mathbb{Z}, A), \Sigma)$ on the set $\mathbb{Z}$ taking values in a ring $A$. It is useful to denote the function $f$ by a matrix

$$
\left[\begin{array}{ccccccc}
\cdots & -2 & -1 & 0 & 1 & 2 & \cdots \\
\cdots & f(-2) & f(-1) & f(0) & f(1) & f(2) & \cdots
\end{array}\right] .
$$

For an element $b$ of a difference algebra $(R, \sigma)$ or a qsi algebra $\left(R, \sigma, \theta^{*}\right)$, we denote by $u[b]$ a function on $\mathbb{Z}$ taking values in the abstract ring $R^{\natural}$ such that

so that

$$
u[b](n)=\sigma^{n}(b) \quad \text { for every } n \in \mathbb{Z}
$$

$$
u[b]=\left[\begin{array}{ccccccc}
\cdots & -2 & -1 & 0 & 1 & 2 & \ldots \\
\cdots & \sigma^{-2}(b) & \sigma^{-1}(b) & b & \sigma^{1}(b) & \sigma^{2}(b) & \ldots
\end{array}\right] .
$$

Therefore $u[b] \in F\left(\mathbb{Z}, R^{\natural}\right)$.

Proposition 3.11 (Heiderich [7, Proposition 2.3.17]). - For a qsi algebra $\left(R, \sigma, \sigma^{-1}, \theta^{*}\right)$, there exists a canonical morphism, which we call the universal Hopf morphism

$$
\begin{aligned}
\iota:\left(R, \sigma, \sigma^{-1}, \theta^{*}\right) & \longrightarrow\left(\left(F\left(\mathbb{Z}, R^{\natural}\right), \Sigma\right)[[X]], \widehat{\Sigma}, \widehat{\Theta}^{*}\right), \\
a & \longmapsto \sum_{i=0}^{\infty} X^{i} u\left[\theta^{(i)}(a)\right]
\end{aligned}
$$

of qsi algebras.

We can also characterize the universal Hopf morphism as the solution of a universal mapping property.

When $q=1$ and $\sigma=\operatorname{Id}_{R}$ and $R$ is commutative so that the qsi ring $\left(R, \operatorname{Id}_{R}, \theta^{*}\right)$ is simply a differential algebra as we have seen in 3.2 .3 and the universal Hopf morphism (3.8) is the universal Taylor morphism in (2.2). Similarly a commutative difference ring is a qsi algebra with trivial derivations as we noticed in 3.2.2. In this case the universal Hopf morphism (3.8) is nothing but the universal Euler morphism (2.20). Therefore the universal Hopf morphism unifies the universal Taylor morphism and the universal Euler morphism.

Let us recall the following fact.

Lemma 3.12. - Let $\left(R, \sigma, \sigma^{-1}, \theta^{*}\right)$ be a qsi domain. If the endomorphism $\sigma: R \rightarrow R$ is an automorphism, then the field $Q(R)$ of fractions of $R$ has the unique structure of qsi field extending that of $R$. 
Proof. - See for example, [6, Proposition 2.5.].

We can interpret the example in 3.2.4 from another viewpoint. We constructed there from a difference ring $(S, \sigma)$ a qsi algebra $\left((S, \sigma)[[X]], \widehat{\Sigma}, \widehat{\Theta}^{*}\right)$. We notice that this procedure is a particular case of Proposition 3.11. In fact, given a difference ring $(S, \sigma)$, as in 3.2.2, by adding the trivial derivations, we get the qsi algebra $\left(S, \sigma, \sigma^{-1}, \theta^{*}\right)$, where

$$
\begin{aligned}
& \theta^{(0)}=\operatorname{Id}_{S}, \\
& \theta^{(i)}=0 \quad \text { for } i \geqslant 1 .
\end{aligned}
$$

Therefore we have the universal Hopf morphism

$$
\left(S, \sigma, \sigma^{-1}, \theta^{*}\right) \longrightarrow\left(F\left(\mathbb{Z}, S^{\natural}\right)[[X]], \widehat{\Sigma}, \widehat{\Theta}^{*}\right)
$$

by Proposition 3.11. So we obtained the $q s i$ algebra $\left(F\left(\mathbb{Z}, S^{\natural}\right)[[X]], \widehat{\Sigma}, \widehat{\Theta}^{*}\right)$ as a result of composite of two functors. Namely,

(1) The functor: (Category of Difference Algebras) $\rightarrow$ (Category of $q s i$ Algebras) of adding trivial derivations

(2) The functor : (Category of qsi Algebras) $\rightarrow$ (Category of qsi Algebras), $A \mapsto B$ if there exists the universal Hopf morphism $A \rightarrow B$.

\subsubsection{Galois hull $\mathcal{L} / \mathcal{K}$ for a qsi field extension}

We can develop a general Galois theory for qsi field extensions analogous to our theories in [23], [24] and [25] thanks to the universal Hopf morphism. Let $L / k$ be an extension of $q s i$ fields such that the abstract field $L^{\natural}$ is finitely generated over the abstract field $k^{\natural}$. Let us assume that we are in characteristic 0 . General theory in [7] works, however, also in characteristic $p \geqslant 0$.

We have by Proposition 3.11 the universal Hopf morphism

$$
\iota:\left(L, \sigma, \sigma^{-1}, \theta^{*}\right) \longrightarrow\left(\left(F\left(\mathbb{Z}, L^{\natural}\right), \Sigma\right)[[X]], \widehat{\Sigma}, \widehat{\Theta}^{*}\right)
$$

so that the image $\iota(L)$ is a copy of the qsi field $L$. We have another copy of $L^{\natural}$. The set

$$
\begin{aligned}
\{f & \left.=\sum_{i=0}^{\infty} X^{i} a_{i} \in F\left(\mathbb{Z}, L^{\natural}\right)[[X]] \mid a_{i}=0 \text { for every } i \geqslant 1 \text { and } \Sigma\left(a_{0}\right)=a_{0}\right\} \\
& =\left\{f \in F\left(\mathbb{Z}, L^{\natural}\right)[[X]] \mid \widehat{\Sigma}(f)=f, \widehat{\Theta}^{(i)}(f)=0 \text { for every } i \geqslant 1\right\}
\end{aligned}
$$

forms the sub-ring of constants in the qsi algebra of the twisted power series

$$
\left(\left(F\left(\mathbb{Z}, L^{\natural}\right), \Sigma\right)[[X]], \widehat{\Sigma}, \widehat{\Theta}^{*}\right) .
$$


We identify $L^{\natural}$ with the ring of constants through the following morphism. For an element $a \in L^{\natural}$, we denote the constant function $f_{a}$ on $\mathbb{Z}$ taking the value $a \in L^{\natural}$ so that

$$
L^{\natural} \longrightarrow\left(\left(F\left(\mathbb{Z}, L^{\natural}\right), \Sigma\right)[[X]], \widehat{\Sigma}, \widehat{\Theta}^{*}\right), \quad a \longmapsto f_{a}
$$

is an injective ring morphism. We may denote the sub-ring in (3.10) by $L^{\natural}$. In fact, as an abstract ring it is isomorphic to the abstract field $L^{\natural}$ and the endomorphism $\widehat{\Sigma}$ and the derivations $\widehat{\Theta}^{(i)},(i \geqslant 1)$ operate trivially on the sub-ring.

We are now exactly in the same situation as in 2.2 .2 of the differential case and in 2.3.2 of the difference case.

We choose a mutually commutative basis $\left\{D_{1}, D_{2}, \ldots, D_{d}\right\}$ of the $L^{\natural}$ vector space $\operatorname{Der}\left(L^{\natural} / k^{\natural}\right)$ of $k^{\natural}$-derivations. So $L^{\sharp}:=\left(L^{\natural},\left\{D_{1}, D_{2}, \ldots, D_{d}\right\}\right)$ is a differential field.

So we introduce derivations $D_{1}, D_{2}, \ldots, D_{d}$ operating on the coefficient ring $F\left(\mathbb{Z}, L^{\natural}\right)$. In other words, we replace the target space $F\left(\mathbb{Z}, L^{\natural}\right)[[X]]$ by $F\left(\mathbb{Z}, L^{\sharp}\right)[[X]]$. Hence the universal Hopf morphism in Proposition 3.11 becomes

$$
\iota: L \longrightarrow F\left(\mathbb{Z}, L^{\sharp}\right)[[X]] .
$$

In the twisted formal power series $\operatorname{ring}\left(F\left(\mathbb{Z}, L^{\sharp}\right)[[X]], \widehat{\Sigma}, \widehat{\Theta}^{*}\right)$, we add differential operators

$$
D_{1}, D_{2}, \ldots, D_{d}
$$

So we have a set $\mathcal{D}$ of the following operators on the $\operatorname{ring}\left(F\left(\mathbb{Z}, L^{\sharp}\right), \Sigma\right)[[X]]$.

(1) The endomorphism $\widehat{\Sigma}$.

$$
\widehat{\Sigma}\left(\sum_{i=0}^{\infty} X^{i} a_{i}\right)=\sum_{i=0}^{\infty} X^{i} q^{i}\left(\Sigma\left(a_{i}\right)\right),
$$

$\Sigma: F\left(\mathbb{Z}, L^{\sharp}\right) \rightarrow F\left(\mathbb{Z}, L^{\sharp}\right)$ being the shift operator of the ring of functions on $\mathbb{Z}$.

(2) The $q$-skew $\widehat{\Sigma}$-derivations $\widehat{\Theta}^{(i)}$ 's in (3.6).

$$
\widehat{\Theta}^{(l)}\left(\sum_{i=0}^{\infty} X^{i} a_{i}\right)=\sum_{i=0}^{\infty} X^{i}\left(\begin{array}{c}
l+i \\
l
\end{array}\right)_{q} a_{i+l} \quad \text { for every } l \in \mathbb{N} .
$$

(3) The derivations $D_{1}, D_{2}, \ldots, D_{d}$ operating through the coefficient $\operatorname{ring} F\left(\mathbb{Z}, L^{\sharp}\right)$ as in $(3.8)$.

Hence we may write $\left(F\left(\mathbb{Z}, L^{\sharp}\right), \mathcal{D}\right)$, where

$$
\mathcal{D}=\left\{\widehat{\Sigma}, D_{1}, D_{2}, \ldots, D_{d}, \widehat{\Theta}^{*}\right\} \quad \text { and } \quad \widehat{\Theta}^{*}=\left\{\widehat{\Theta}^{(i)}\right\}_{i \in \mathbb{N}} .
$$


We identify using inclusion (3.11)

$$
L^{\sharp} \longrightarrow F\left(\mathbb{Z}, L^{\sharp}\right)[[X]] .
$$

We sometimes denote the image $f_{a}$ of an element $a \in L^{\sharp}$ by $a^{\sharp}$.

We are ready to define Galois hull as in Definition 2.1.

Definition 3.13. - The Galois hull $\mathcal{L} / \mathcal{K}$ is a $\mathcal{D}$-invariant sub-algebra of $F\left(\mathbb{Z}, L^{\sharp}\right)[[X]]$, where $\mathcal{L}$ is the $\mathcal{D}$-invariant sub-algebra generated by the image $\iota(L)$ and $L^{\sharp}$ and $\mathcal{K}$ is the $\mathcal{D}$-invariant sub-algebra generated by the image $\iota(k)$ and $L^{\sharp}$. So $\mathcal{L} / \mathcal{K}$ is a $\mathcal{D}$-algebra extension.

As in 2.4, if we have to emphasize that we deal with qsi algebras, we denote the Galois hull by $\mathcal{L}_{\sigma \theta} / \mathcal{K}_{\sigma \theta}$.

We notice that we are now in a totally new situation. In the differential case, the universal Taylor morphism maps the given fields to the commutative algebra of the formal power series ring so that the Galois hull is an extension of commutative algebras. The situation is similar for the universal Euler morphism of difference rings. The commutativity of the Galois hull comes from the fact in the differential and difference cases, the theory depends on the co-commutative Hopf algebras. When we treat the qsi algebras, the Hopf algebra $\mathcal{H}_{q}$ is not co-commutative so that the Galois hull $\mathcal{L} / \mathcal{K}$ is included in the non-commutative algebra of twisted formal power series algebra, the dual algebra of $\mathcal{H}_{q}$. So even if we start from a (commutative) field extension $L / k$, the Galois hull can be non-commutative. See the examples in Sections 4,5 and 6 . We also notice that when $L / k$ is a Picard-Vessiot extension of qsi algebras, the Galois hull is commutative [26].

As the Galois hull is non-commutative, if we limit ourselves to the category of commutative $L^{\natural}$-algebras ( $\mathrm{Alg} / L^{\natural}$ ), we can not detect non-commutative nature of the qsi field extension. So it is quite natural to extend the functors onto the category of not necessarily commutative algebras.

\subsubsection{Infinitesimal deformation functor $\mathcal{F}_{L / k}$ for a $q s i$ field exten- sion}

We pass to the task of defining the infinitesimal deformation functor $\mathcal{F}_{L / k}$ and the Galois group functor. The latter is a subtle object and we postpone discussing it until Section 7.

Instead we define naively the infinitesimal automorphism functor $\operatorname{Inf-Gal}(L / k)$, which does not seem useful in general. 
We have the universal Taylor morphism

$$
\iota_{L^{\sharp}}: L^{\sharp} \longrightarrow\left(L^{\natural}\left[\left[W_{1}, W_{2}, \ldots, W_{d}\right]\right],\left\{\frac{\partial}{\partial W_{1}}, \frac{\partial}{\partial W_{2}}, \ldots, \frac{\partial}{\partial W_{d}}\right\}\right)
$$

as in (2.6). So by (3.12), we have the canonical morphism

$$
\left(F\left(\mathbb{Z}, L^{\sharp}\right)[[X]], \mathcal{D}\right) \longrightarrow\left(F\left(\mathbb{Z}, L^{\natural}[[W]]\right)[[X]], \mathcal{D}\right),
$$

where in the target space

$$
\mathcal{D}=\left\{\widehat{\Sigma}, \frac{\partial}{\partial W_{1}}, \frac{\partial}{\partial W_{2}}, \ldots, \frac{\partial}{\partial W_{d}}, \widehat{\Theta}^{*}\right\}
$$

by abuse of notation.

For an $L^{\natural}$-algebra $A$, the structure morphism $L^{\natural} \rightarrow A$ induces the canonical morphism

$$
\left(F\left(\mathbb{Z}, L^{\natural}[[W]]\right)[[X]], \mathcal{D}\right) \longrightarrow(F(\mathbb{Z}, A[[W]])[[X]], \mathcal{D}) .
$$

The composite of the $\mathcal{D}$-morphisms (3.13) and (3.14) gives us the canonical morphism

$$
\left(F\left(\mathbb{Z}, L^{\sharp}\right)[[X]], \mathcal{D}\right) \longrightarrow(F(\mathbb{Z}, A[[W]])[[X]], \mathcal{D}) .
$$

The restriction of the morphism (3.15) to the $\mathcal{D}$-invariant sub-algebra $\mathcal{L}$ gives us the canonical morphism

$$
\iota:(\mathcal{L}, \mathcal{D}) \longrightarrow(F(\mathbb{Z}, A[[W]])[[X]], \mathcal{D}) .
$$

We can define the functor exactly as in Paragraphs 2.2.4 for the differential case and 2.3.3 for the difference case.

DeFinition 3.14 (Introductory definition). - We define the functor

$$
\mathcal{F}_{L / k}:\left(\mathrm{Alg} / L^{\natural}\right) \longrightarrow(\mathrm{Set})
$$

from the category (Alg / $L^{\natural}$ ) of commutative $L^{\natural}$-algebras to the category (Set) of sets, by associating to an $L^{\natural}$-algebra $A$, the set of infinitesimal deformations of the canonical morphism (3.15).

Hence

$$
\begin{aligned}
& \mathcal{F}_{L / k}(A) \\
& =\left\{\begin{array}{l|l}
f:(\mathcal{L}, \mathcal{D}) & \begin{array}{l}
f \text { is an algebra morphism compatible } \\
\text { with } \mathcal{D}, \text { congruent to the canonical } \\
\text { morphism } \iota \text { modulo nilpotent elements } \\
\text { such that } f=\iota \text { when restricted to the } \\
\text { sub-algebra } \mathcal{K}
\end{array}
\end{array}\right\} .
\end{aligned}
$$

The introductory definition 3.14 is exact, analogous to Definitions in 2.2.4 and 2.3.3 and easy to understand. As we explained in 3.2.7, we, however, have to consider also deformations over non-commutative algebras. 
We have to treat both the category of commutative $L^{\natural}$-algebras and that of not necessarily commutative $L^{\natural}$-algebras.

DeFINITIOn 3.15. - All the associative algebras that we consider are unitary and the morphisms between them are assumed to be unitary. For a commutative algebra $R$, we denote by $(\mathrm{CAlg} / R)$ the category of associative commutative $R$-algebras. We consider also the category $(\mathrm{NCAlg} / R$ ) of not necessarily commutative $R$-algebras $A$ such that (the image in $A$ of) $R$ is in the center of $A$. When there is no danger of confusion the category of commutative algebras is denoted simply by $(\mathrm{Alg} / R)$.

Let us come back to the qsi field extension $L / k$. We can now give the infinitesimal deformation functors in an appropriate language.

Definition 3.16. - The functor $\mathcal{F}_{L / k}$ defined in 3.14 will be denoted by $\mathcal{C F}_{L / k}$. So we have

$$
\mathcal{C F}_{L / k}:\left(\mathrm{CAlg} / L^{\natural}\right) \longrightarrow(\text { Set }) .
$$

We extend formally the functor $\mathcal{C F}_{L / k}$ in 3.14 from the category $\left(\mathrm{CAlg} / L^{\natural}\right)$ to the category (NCAlg $\left./ L^{\natural}\right)$. Namely, we define the functor

$$
\mathcal{N C F}_{L / k}:\left(\mathrm{NCAlg} / L^{\natural}\right) \longrightarrow(\text { Set })
$$

by setting

$\mathcal{F}_{L / k}(A)$

$=\left\{\begin{array}{l|l}f:(\mathcal{L}, \mathcal{D}) & \begin{array}{l}f \text { is an algebra morphism compatible with } \\ \mathcal{D}, \text { congruent to the canonical morphism } \iota \\ \text { modulo nilpotent elements such that } f=\iota \\ \text { when restricted to the sub-algebra } \mathcal{K}\end{array}\end{array}\right\}$

for $A \in \mathrm{ob}\left(\mathrm{NCAlg}_{L / k}\right)$.

In the examples, we consider qsi structure, differential structure and difference structure of a given field extension $L / k$ and we study Galois groups with respect to the structures. So we have to clarify which structure is in question. For this reason, when we treat qsi structure, we sometimes add suffix $\sigma \theta^{*}$ to indicate that we treat the qsi structure as in 2.4. For example $\mathcal{N C F}_{\sigma \theta^{*} L / k}$.

\subsubsection{Definition of commutative Galois group functor CInf-Gal $(L / k)$}

Similarly to the Galois group functor $\operatorname{Inf}-\operatorname{Gal}(L / k)$ in the differential and the difference cases, we may introduce the group functor CInf-Gal $(L / k)$ called commutative Galois group functor, on the category $\left(\mathrm{CAlg} / L^{\natural}\right)$. 
Definition 3.17. - In the differential case and in the difference case, the Galois group in our Galois theory is the group functor

$$
\operatorname{CInf-Gal}(L / k):\left(\mathrm{CAlg} / L^{\natural}\right) \longrightarrow(G r p)
$$

defined by

$$
\begin{aligned}
& \operatorname{CInf-Gal}(L / k)(A)
\end{aligned}
$$

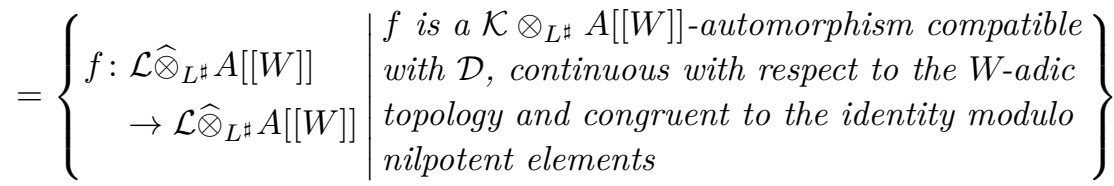

for a commutative $L^{\natural}$-algebra A. See [16, Definition 2.19.].

Then the group functor $\operatorname{CInf-Gal}(L / k)$ would operate on the functor $\mathcal{C F}_{L / k}$ in such a way that the operation $\left(\operatorname{CInf}-\operatorname{Gal}(L / k), \mathcal{F}_{L / k}\right)$ is a torsor.

Remark 3.18. - For a qsi field extension $L / k$, the Galois hull $\mathcal{L} / \mathcal{K}$ is, in general, a non-commutative algebra extension so that the commutative Galois group functor CInf-Gal $(L / k)$ on the category $\left(\mathrm{CAlg} / L^{\natural}\right)$ is not adequate for the following two reasons.

(1) If we measure the extension $\mathcal{L} / \mathcal{K}$ over the category $\left(\mathrm{CAlg} / L^{\natural}\right)$ by the commutative Galois group functor $\operatorname{CInf-Gal}(L / k)$, the noncommutative data of the extension $\mathcal{L} / \mathcal{K}$ are lost.

(2) We hope to get a quantum group as a Galois group. A quantum group is, however, in any sense not a group functor on the category (NCAlg $/ L^{\natural}$ ) of non-commutative $L^{\natural}$-algebras.

In the three coming sections, we settle these points for three concrete examples. Looking at these examples, we are led to a general definition in Section 7. The idea is to look at the coordinate transformations of initial conditions. As it is easier to understand it with examples, we explain the definition there. See Questions 7.1.

\section{The First Example, the $q s i$ field extension$$
L / k=\left(\mathbb{C}(t), \sigma, \sigma^{-1}, \theta^{*}\right) / \mathbb{C}
$$

From now on, we assume $C=\mathbb{C}$. The arguments below work for an algebraically closed field $C$ of characteristic 0 . So $q$ is a non-zero complex number. 


\subsection{Analysis of the example}

Let $t$ be a variable over $\mathbb{C}$. The field $\mathbb{C}(t)$ of rational functions has various structures: the differential field structure, the $q$-difference field structure and the qsi field structure that we are going to define. We are interested in the Galois group of the field extension $\mathbb{C}(t) / \mathbb{C}$ with respect to these structures. Let $\sigma: \mathbb{C}(t) \rightarrow \mathbb{C}(t)$ be the $\mathbb{C}$-automorphism of the rational function field $\mathbb{C}(t)$ sending $t$ to $q t$. So $(\mathbb{C}(t), \sigma)$ is a difference field. We assume $q^{n} \neq 1$ for every positive integer $n$. We define a $\mathbb{C}$-linear map $\theta^{(1)}: \mathbb{C}(t) \rightarrow \mathbb{C}(t)$ by

$$
\theta^{(1)}(f(t)):=\frac{\sigma(f)-f}{\sigma(t)-t}=\frac{f(q t)-f(t)}{(q-1) t} \quad \text { for } f(t) \in \mathbb{C}(t) .
$$

For an integer $n \geqslant 2$, we set

$$
\theta^{(n)}:=\frac{1}{[n]_{q} !}\left(\theta^{(1)}\right)^{n} .
$$

It is convenient to define

$$
\theta^{(0)}=\operatorname{Id}_{\mathbb{C}(t)} .
$$

It is well-known and easy to check that $\left(\mathbb{C}(t), \sigma, \sigma^{-1}, \theta^{*}\right)=\left(\mathbb{C}(t), \sigma, \sigma^{-1}\right.$, $\left.\left\{\theta^{(i)}\right\}_{i \in \mathbb{N}}\right)$ is a qsi algebra.

We have to clarify a notation. For an algebra $R$, a sub-algebra $S$ of $R$ and a sub-set $T$ of $R$, we denote by $S\langle T\rangle_{\text {alg }}$ the sub-algebra of $R$ generated over $S$ by $T$.

Lemma 4.1. - The difference field extension $(\mathbb{C}(t), \sigma) /\left(\mathbb{C}, \operatorname{Id}_{\mathbb{C}}\right)$ is a Picard-Vessiot extension. Its Galois group is the multiplicative group $\mathbb{G}_{m \mathbb{C}}$.

Proof. - Since $t$ satisfies the linear difference equation $\sigma(t)=q t$ over $\mathbb{C}$ and the field $C_{\mathbb{C}(t)}$ of constant of $\mathbb{C}(t)$ is $\mathbb{C}$, the extension $(\mathbb{C}(t), \sigma) /\left(\mathbb{C}, \operatorname{Id}_{\mathbb{C}}\right)$ is a difference Picard-Vessiot extension. The result follows from the definition of the Galois group.

When $q \rightarrow 1$, the limit of the $q s i \operatorname{ring}\left(\mathbb{C}(t), \sigma, \sigma^{-1}, \theta^{*}\right)$ is the differential algebra $(\mathbb{C}(t), \mathrm{d} / \mathrm{d} t)$. We denote by $A F_{1 k}$, the algebraic group of affine transformations of the affine line so that

$$
A F_{1 \mathbb{C}}=\left\{\left[\begin{array}{ll}
a & b \\
0 & 1
\end{array}\right] \mid a, b \in \mathbb{C}, a \neq 0\right\} .
$$

Then

$$
\begin{aligned}
A F_{1 \mathbb{C}} & \simeq \mathbb{G}_{m \mathbb{C}} \ltimes \mathbb{G}_{a \mathbb{C}}, \\
& -1350-
\end{aligned}
$$


where

$$
\begin{aligned}
& \mathbb{G}_{m \mathbb{C}} \simeq\left\{\left[\begin{array}{ll}
a & 0 \\
0 & 1
\end{array}\right] \in A F_{1 \mathbb{C}} \mid a \in \mathbb{C}^{*}\right\}, \\
& \mathbb{G}_{a \mathbb{C}} \simeq\left\{\left[\begin{array}{ll}
1 & b \\
0 & 1
\end{array}\right] \in A F_{1 \mathbb{C}} \mid b \in \mathbb{C}\right\} .
\end{aligned}
$$

LEMmA 4.2. - The Galois group of differential Picard-Vessiot extension $(\mathbb{C}(t), \mathrm{d} / \mathrm{d} t) / \mathbb{C}$ is $\mathbb{G}_{a \mathbb{C}}$.

Proof. - We consider the linear differential equation

$$
Y^{\prime}=\left[\begin{array}{ll}
0 & 1 \\
0 & 0
\end{array}\right] Y,
$$

where $Y$ is a $2 \times 2$-matrix with entries in a differential extension field of $\mathbb{C}$. Then $\mathbb{C}(t) / \mathbb{C}$ is the Picard-Vessiot extension for (4.1),

$$
Y=\left[\begin{array}{ll}
1 & t \\
0 & 1
\end{array}\right]
$$

being a fundamental solution of (4.1). The result is well-known and follows from, the definition of Galois group.

The $q s i$ field extension $\left(\mathbb{C}(t), \sigma, \sigma^{-1}, \theta^{*}\right) / \mathbb{C}$ is not a Picard-Vessiot extension in the sense of Hardouin [5] and Masuoka and Yanagawa [14] so that we can not treat it in the framework of Picard-Vessiot theory. We can apply, however, Hopf Galois theory of Heiderich [7].

Proposition 4.3. - The commutative Galois group CInf-Gal $((\mathbb{C}(t), \sigma$, $\left.\left.\sigma^{-1}, \theta^{*}\right) / \mathbb{C}\right)$ of the extension $\left(\mathbb{C}(t), \sigma, \sigma^{-1}, \theta^{*}\right) / \mathbb{C}$ is isomorphic to the formal completion $\widehat{\mathbb{G}}_{m \mathbb{C}}$ of the multiplicative group $\mathbb{G}_{m \mathbb{C}}$.

Proof. - Before we start the proof the Proposition, we observe the behaviors of the Galois group under specializations. Theories of Umemura [22] and Heiderich [7] single out only the Lie algebra. Proposition 4.3 should be understood in the following manner. We have two specializations of the qsi field extension $\left(\mathbb{C}(t), \sigma, \sigma^{-1}, \theta^{*}\right) / \mathbb{C}$.

(i) Forgetting $\theta^{*}$, or equivalently specializing

$$
\theta^{(i)} \longrightarrow 0 \text { for } i \geqslant 1 \text {, }
$$

we get the difference field extension $(\mathbb{C}(t), \sigma) / \mathbb{C}$. See 3.2.2.

(ii) The specialization $q \rightarrow 1$ gives the differential field extension $(\mathbb{C}(t), \mathrm{d} / \mathrm{d} t) / \mathbb{C}$. See 2.2 .2 .

We can summarize the behavior of the Galois group under the specializations. 
(1) Proposition 4.3 says that the commutative Galois group

$$
\mathrm{C} \text { Inf-Gal }{ }_{\sigma, \sigma^{-1}, \theta^{*}}(L / k)
$$

of $\left(\mathbb{C}(t), \sigma, \sigma^{-1}, \theta^{*}\right) / \mathbb{C}$ is the formal completion $\widehat{\mathbb{G}}_{m \mathbb{C}}$ of the multiplicative group $\mathbb{G}_{m \mathbb{C}}$.

(2) By Lemma 4.1, the Galois group of the specialization (ii) is the formal completion $\widehat{\mathbb{G}}_{a \mathbb{C}}$ of the additive group $\mathbb{G}_{a \mathbb{C}}$.

(3) The Galois group of the specialization (ii) is the formal completion $\widehat{\mathbb{G}}_{a \mathbb{C}}$ of the additive group $\mathbb{G}_{a \mathbb{C}}$ by Lemma 4.2 .

Now let us come back to the proof of the Proposition. Let us set $L=$ $\left(\mathbb{C}(t), \sigma, \sigma^{-1}, \theta^{*}\right)$ and $k=\left(\mathbb{C}, \sigma, \sigma^{-1}, \theta^{*}\right)$. By definition of the universal Hopf morphism (3.8),

$$
\begin{aligned}
\iota:\left(L, \sigma, \sigma^{-1}, \theta^{*}\right) & \longrightarrow\left(F\left(\mathbb{Z}, L^{\natural}\right)[[X]], \widehat{\Sigma}, \widehat{\Theta}^{*}\right), \\
t & \longmapsto t Q+X \in F\left(\mathbb{Z}, L^{\natural}\right)[[X]],
\end{aligned}
$$

where $Q \in F\left(\mathbb{Z}, L^{\natural}\right)$ is the function on $\mathbb{Z}$ with values in $\mathbb{C} \subset L^{\natural}$ defined by

$$
Q(n)=q^{n} \quad \text { for } n \in \mathbb{Z} .
$$

We denote the function $Q$ by the matrix

$$
Q=\left[\begin{array}{ccccccc}
\cdots & -2 & -1 & 0 & 1 & 2 & \cdots \\
\cdots & q^{-2} & q^{-1} & 1 & q & q^{2} & \cdots
\end{array}\right]
$$

according to the convention. We take the derivation $\partial / \partial t \in \operatorname{Der}\left(L^{\natural} / k^{\natural}\right)$ as a basis of the 1-dimensional $L^{\natural}$-vector space $\operatorname{Der}\left(L^{\natural} / k^{\natural}\right)$ of $k^{\natural}$-derivations of $L^{\natural}$. So $(\partial / \partial t)(\iota(t))=Q$ is an element of the Galois hull $\mathcal{L}$. Therefore

$$
\mathcal{L} \supset \mathcal{L}^{o}:=L^{\sharp}\langle X, Q\rangle_{\text {alg }},
$$

which is the $L^{\sharp}$-sub-algebra of $F\left(\mathbb{Z}, L^{\sharp}\right)[[X]]$ generated by $X$ and $Q$. So the algebra $\mathcal{L}^{o}$ is invariant under $\widehat{\Sigma}, \widehat{\Theta}^{*}$ and $\partial / \partial t$. Since $Q X=q X Q$, the Galois hull $\mathcal{L}$ is a non-commutative $L^{\natural}$-algebra. Now we consider the universal Taylor expansion

$$
\left(L^{\natural}, \partial / \partial t\right) \longrightarrow L^{\natural}[[W]]
$$

and consequently we get the canonical morphism

$$
\iota: \mathcal{L} \longrightarrow F\left(\mathbb{Z}, L^{\sharp}\right)[[X]] \longrightarrow F\left(\mathbb{Z}, L^{\natural}[[W]]\right)[[X]] .
$$

We study infinitesimal deformations of $\iota$ in $(4.2)$ over the category $\left(\mathrm{CAlg} / L^{\natural}\right)$ of commutative $L^{\natural}$-algebras. Let $A$ be a commutative $L^{\natural}$-algebra and

$$
\varphi: \mathcal{L} \longrightarrow F(\mathbb{Z}, A[[W]])[[X]]
$$

be an infinitesimal deformation of the canonical morphism

$$
\iota: \mathcal{L} \longrightarrow F(\mathbb{Z}, A[[W]])[[X]] .
$$


Sub-LEMmA 4.4. - We keep the notation above.

(1) There exists a nilpotent element $n \in A$ such that $\varphi(Q)=(1+n) Q$ and $\varphi(X)=X$.

(2) The commutative infinitesimal deformation $\varphi$ is determined by the nilpotent element $n$ such that $\varphi(Q)=(1+n) Q$.

(3) Conversely, for every nilpotent element $n \in A$, there exists a unique commutative infinitesimal deformation $\varphi_{e} \in \mathcal{F}_{L / k}(A)$ such that $\varphi_{e}(Q)=e Q$, where we set $e=1+n$.

Sublemma proves Proposition 4.3.

Proof of Sublemma. The elements $X, Q \in \mathcal{L}$ satisfy the following equation.

$$
\begin{aligned}
\frac{\partial X}{\partial W} & =\frac{\partial Q}{\partial W}=0, \\
\widehat{\Sigma}(X) & =q X, \quad \widehat{\Sigma}(Q)=q Q, \\
\widehat{\Theta}^{(1)}(X) & =1, \quad \widehat{\Theta}^{(i)}(X)=0 \quad \text { for } i \geqslant 2, \\
\widehat{\Theta}^{(i)}(Q) & =0 \quad \text { for } i \geqslant 1 .
\end{aligned}
$$

So $\varphi(X), \varphi(Q)$ satisfy the same equations as above, which shows

$$
\begin{aligned}
& \varphi(X)=X+f Q \in F(\mathbb{Z}, A[[W]])[[X]], \\
& \varphi(Q)=e Q \in F(\mathbb{Z}, A[[W]])[[X]],
\end{aligned}
$$

where $f, e \in A$. Since $\varphi$ is an infinitesimal deformation of $\iota, f$ and $e-1$ are nilpotent elements in $A$. We show the first $f=0$. In fact, it follows from the equation

$$
Q X=q X Q
$$

that

$$
\varphi(Q) \varphi(X)=q \varphi(X) \varphi(Q)
$$

or

$$
e Q(X+f Q)=q(X+f Q) e Q .
$$

So we have

$$
e Q f Q=q f Q e Q
$$

and so

Therefore

$$
\text { ef } Q^{2}=q f e Q^{2}
$$

$$
e f=q f e .
$$

Since $e$ is a unit, $e-1$ being nilpotent in $A$,

$$
f-q f=0,
$$

so that

$$
\begin{gathered}
(1-q) f=0 . \\
-1353-
\end{gathered}
$$


As $1-q$ is a non-zero complex number, $f=0$. So we proved (1). In other words, we determined the restriction of $\varphi$ to the sub-algebra $\mathcal{L}^{o}=$ $L^{\sharp}\langle X, Q\rangle_{\text {alg }} \subset \mathcal{L}$. To prove (2), we have to show that $\varphi$ is determined by its restriction on $\mathcal{L}^{o}$. To this end, we take two commutative infinitesimal deformations $\varphi, \psi \in \mathcal{F}_{L / k}(A)$ such that

$$
\varphi(Q)=e Q \quad \text { and } \quad \psi(Q)=e Q,
$$

where $n$ is a nilpotent element in $A$ and we set $e=1+n$. Since

$$
\mathcal{L}=L^{\sharp} . \iota(\mathbb{C}(t))\langle X, Q\rangle_{\text {alg }}=L^{\sharp}\left\langle X, Q, \iota\left((t+c)^{-1}\right)\right\rangle_{c \in \mathbb{C} \text { alg }},
$$

and since $\varphi$ is a $\mathcal{K}=L^{\sharp}$-morphism, it is sufficient to show that

$$
\varphi\left((t+c)^{-1}\right)=\psi\left((t+c)^{-1}\right)
$$

for every complex number $c \in \mathbb{C}$. Since $\iota(t+c) \in \mathcal{L}^{o}, \varphi(t+c)=\psi(t+c)$ and so

$$
\varphi\left((t+c)^{-1}\right)=\varphi(t+c)^{-1}=\psi(t+c)^{-1}=\psi\left((t+c)^{-1}\right) .
$$

This is what we had to show.

Now we prove (3). We introduce another sub-algebra by

$$
\widetilde{\mathcal{L}}:=\left\{\sum_{n=0}^{\infty} X^{n} a_{n} \in F\left(\mathbb{Z}, L^{\sharp}\right)[[X]] \mid a_{n} \in L^{\sharp}(Q) \text { for every } n \in \mathbb{N}\right\} .
$$

By commutation relation (3.4), this $\widetilde{\mathcal{L}}$ is indeed a sub-algebra of $F\left(\mathbb{Z}, L^{\sharp}\right)[[X]]$, and is invariant under $\widehat{\Sigma}, \widehat{\Theta}^{*}$ and the derivation $\partial / \partial t$. We show $\mathcal{L} \subset \widetilde{\mathcal{L}}$. Since the sub-algebra $\mathcal{L}$ is generated by $\iota(L)$ and $L^{\sharp}$ along with operators $\widehat{\Sigma}, \widehat{\Theta}^{*}$ and $\partial / \partial t$. So it is sufficient to prove $L^{\sharp}$ and $\iota(L)$ are sub-algebras of $\widetilde{\mathcal{L}}$. The first inclusion $L^{\sharp} \subset \widetilde{\mathcal{L}}$ being trivial, it remains to show the second inclusion:

$$
\iota(L)=\iota(\mathbb{C}(t)) \subset \widetilde{\mathcal{L}} .
$$

We have to show that (i) $\iota(t) \in \widetilde{\mathcal{L}}$, and (ii) $\iota(t+c)^{-1} \in \widetilde{\mathcal{L}}$ for every complex number $c \in \mathbb{C}$. The first assertion (i) follows from the equality $\iota(t)=t Q+X$. As for the assertion (ii), we notice

$$
\begin{aligned}
\iota\left((t+c)^{-1}\right) & =\iota(t+c)^{-1} \\
& =(t Q+X+c)^{-1} \\
& =(t Q+c)^{-1}\left(1+(t Q+c)^{-1} X\right)^{-1} \\
& =(t Q+c)^{-1}(1-A)^{-1} \\
& =(t Q+c)^{-1} \sum_{n=0}^{\infty} A^{n},
\end{aligned}
$$


where we set $A=-(t Q+c)^{-1} X$. Upon writing $a(Q):=-(t Q+c)^{-1}$, we have

$$
A=X a(q Q), \quad A^{2}=X^{2} a(q Q) a\left(q^{2} Q\right), \ldots, A^{n}=X^{n} \prod_{i=1}^{n} a\left(q^{i} Q\right), \ldots
$$

by commutation relation (3.4). Hence, by $(4.3), \iota(t+c)^{-1} \in \widetilde{\mathcal{L}}$. Thus we proved the inclusion $\mathcal{L}^{o} \subset \widetilde{\mathcal{L}}$.

To complete the proof of (3), a nilpotent element $n$ of the algebra $A$ being given, we set $e=1+n$. As we have $q X e Q=e Q X$, by the commutation relation (3.4), there exists an infinitesimal deformation

$$
\psi_{e}: \widetilde{\mathcal{L}} \longrightarrow F(\mathbb{Z}, A[[W]])[[X]]
$$

such that $\psi_{e}(X)=X$ and $\psi_{e}(Q)=e Q$ and continuous with respect to the $X$-adic topology. Therefore to be more concrete $\psi_{e}$ maps an element

$$
\sum_{n=0}^{\infty} X^{n} a_{n}(Q) \quad \text { with } a_{n}(Q) \in L^{\sharp}(Q) \text { for every } n \in \mathbb{N}
$$

of the algebra $\widetilde{\mathcal{L}}$ to an element

$$
\sum_{n=0}^{\infty} X^{n} a_{n}(e Q) \in F(\mathbb{Z}, A[[W]])[[X]] .
$$

If we denote the restriction $\left.\psi_{e}\right|_{\mathcal{L}}$ to $\mathcal{L}$ by $\varphi_{e}$, then $\varphi_{e}$ satisfies all the required conditions except for the uniqueness. The uniqueness follows from (2) that we have already proved above.

We have shown that the functor

$$
\mathcal{F}_{L / k}:\left(\mathrm{Alg} / L^{\natural}\right) \rightarrow(\mathrm{Set})
$$

is a torsor of the group functor $\widehat{\mathbb{G}}_{m \mathbb{C}}$. For origin of the group structure, see Paragraph 2.2.6 as well as Paragraph 4.3.1 below.

In the course of the proof of Proposition 4.3, we have proved the following

Proposition 4.5. - The Galois hull $\mathcal{L}$ coincides with the sub-algebra

$$
L^{\sharp}\left\langle X, Q,(c+t Q+X)^{-1}\right\rangle_{c \in \mathbb{C} \text { alg }}
$$

of $F\left(\mathbb{Z}, L^{\sharp}\right)[[X]]$ generated by $L^{\sharp}, X, Q$ and the set $\left\{(c+t Q+X)^{-1} \mid c \in \mathbb{C}\right\}$. The commutation relation of $X$ and $Q$ is

$$
Q X=q X Q .
$$

In particular, if $q \neq 1$, then the Galois hull is non-commutative. 


\subsection{Non-commutative deformation functor $\mathcal{N C F}_{L / k}$ for $L / k=$} $\left(\mathbb{C}(t), \sigma, \sigma^{-1}, \theta^{*}\right) / \mathbb{C}$

We are ready to describe the non-commutative deformations. Let $A \in$ $\mathrm{ob}\left(\mathrm{NCAlg} / L^{\natural}\right)$.

Lemma 4.6. - If $q \neq 1$, we have

$$
\mathcal{N C F}_{L / k}(A)=\left\{(e, f) \in A^{2} \mid q f e=e f \text { and } e-1, f \text { are nilpotent }\right\}
$$

for every $A \in \mathrm{ob}\left(\mathrm{NCAlg} / L^{\natural}\right)$.

Proof. - Since $q \neq 1$, it follows from the argument of the proof of Sublemma 4.4 that if we take

$$
\varphi \in \mathcal{N C F}_{L / k}(A) \quad \text { for } A \in \mathrm{ob}\left(\mathrm{NCAlg} / L^{\natural}\right),
$$

then $\varphi(X)=X+f Q$ and $\varphi(Q)=e Q$ for $f, e \in A$.

Since $\varphi$ is an infinitesimal deformation of $\iota, f$ and $e-1$ are nilpotent.

It follows from $Q X=q X Q$ that

$$
e Q(X+f Q)=q(X+f Q) e Q
$$

so $e f=q f e$.

Suppose conversely that elements $e, f \in A$ such that $e-1, f$ are nilpotent and such that $e f=q f e$ are given. Then the argument of the proof of Sublemma 4.4 allows us to show the unique existence of the infinitesimal deformation $\varphi \in \mathcal{N C F}_{L / k}(A)$ such that

$$
\varphi(X)=X+f Q, \varphi(Q)=e Q .
$$

We are going to see in 4.3.1 that theoretically, we can identify

$$
\mathcal{N C F}_{L / k}(A)=\left\{\left[\begin{array}{ll}
e & f \\
0 & 1
\end{array}\right] \mid e, f \in A, \text { qfe }=\text { ef } \text { and } e-1, f \text { are nilpotent }\right\} \text {. }
$$

Corollary 4.7 (Corollary to the proof of Lemma 4.6). - When $q=$ 1 that is the case excluded in our general study, we consider the qsi field extension

$$
\left(\mathbb{C}(t), \operatorname{Id}, \theta^{*}\right) / \mathbb{C}
$$

as in 3.2.3. So $\theta^{*}$ is the iterative derivation;

$$
\theta^{(0)}=\mathrm{Id}, \quad \theta^{(i)}=\frac{1}{i !} \frac{d^{i}}{d t^{i}} \quad \text { for } i \geqslant 1 .
$$


Then we have

$$
\begin{aligned}
& \mathcal{L}_{\mathrm{Id}, \theta^{*}} \simeq \mathcal{L}_{\mathrm{d} / \mathrm{d} t}, \\
& \mathcal{N C F}_{\left(\mathbb{C}(t), \operatorname{Id}, \theta^{*}\right) / \mathbb{C}}(A)=\{f \in A \mid f \text { is a nilpotent element }\}
\end{aligned}
$$

for $A \in \mathrm{ob}\left(\mathrm{NCAlg} / L^{\natural}\right)$.

Proof. - In fact, if $q=1$, then

$$
Q=\left[\begin{array}{ccccccc}
\cdots & -2 & -1 & 0 & 1 & 2 & \ldots \\
\cdots & 1 & 1 & 1 & 1 & 1 & \ldots
\end{array}\right]=1 \in \mathbb{C} .
$$

So $\mathcal{L}_{\mathrm{Id}, \theta^{*}}$ is generated by $X$ over $\mathcal{K}$. Therefore $\mathcal{L}_{\mathrm{Id}, \theta^{*}} \simeq \mathcal{L}_{\mathrm{d} / \mathrm{d} t}$. Since $Q=1 \in$ $\mathcal{K}, \varphi(Q)=Q$ for an infinitesimal deformation

$$
\varphi \in \mathcal{N C F}_{\left(\mathbb{C}(t), \operatorname{Id}, \theta^{*}\right) / \mathbb{C}}(A)
$$

and we get (4.6).

\subsubsection{Quantum group enters}

To understand Lemma 4.6, it is convenient to introduce a quantum group.

Definition 4.8. - Let $A \in \operatorname{ob}(\mathrm{NCAlg} / \mathbb{C})$. We say that two sub-sets $S, T$ of $A$ are element-wisely commutative if for every $s \in S, t \in T$, we have $[s, t]=s t-t s=0$.

For $A \in \mathrm{ob}\left(\mathrm{NCAlg} / L^{\natural}\right)$, we set

$$
H_{q}(A)=\left\{\left[\begin{array}{ll}
e & f \\
0 & 1
\end{array}\right] \mid e, f \in A, e \text { is invertible in } A, \text { ef }=q f e\right\} .
$$

LEMMA 4.9. - Given two matrices

$$
Z_{1}=\left[\begin{array}{cc}
e_{1} & f_{1} \\
0 & 1
\end{array}\right], \quad Z_{2}=\left[\begin{array}{cc}
e_{2} & f_{2} \\
0 & 1
\end{array}\right] \in H_{q}(A),
$$

suppose that $\left\{e_{1}, f_{1}\right\}$ and $\left\{e_{2}, f_{2}\right\}$ are element-wisely commutative. Then we have

$$
Z_{1} Z_{2} \in H_{q}(A)
$$

Proof. - Since

we have to prove

$$
Z_{1} Z_{2}=\left[\begin{array}{cc}
e_{1} e_{2} & e_{1} f_{2}+f_{1} \\
0 & 1
\end{array}\right]
$$

$$
e_{1} e_{2}\left(e_{1} f_{2}+f_{1}\right)=q\left(e_{1} f_{2}+f_{1}\right) e_{1} e_{2} .
$$

This follows from the element-wise commutativity of $\left\{e_{1}, f_{1}\right\}$ and $\left\{e_{2}, f_{2}\right\}$, and from the conditions $e_{1} f_{1}=q f_{1} e_{1}, e_{2} f_{2}=q f_{2} e_{2}$. 
Lemma 4.10. - Given a matrix

$$
Z=\left[\begin{array}{ll}
e & f \\
0 & 1
\end{array}\right] \in H_{q}(A),
$$

set

Then we have

$$
\widetilde{Z}=\left[\begin{array}{cc}
e^{-1} & -e^{-1} f \\
0 & 1
\end{array}\right] \in M_{2} .
$$

$$
\widetilde{Z} \in H_{q^{-1}}(A) \text { and } \widetilde{Z} Z=Z \widetilde{Z}=I_{2} .
$$

Proof. - We can check it by a simple calculation. See also Remark 4.11, where the first assertion is proved.

Remark 4.11. - If $q^{2} \neq 1$, for $f \neq 0, \widetilde{Z} \notin H_{q}(A)$. In fact, let us set

$$
\widetilde{Z}=\left[\begin{array}{ll}
\widetilde{e} & \widetilde{f} \\
0 & 1
\end{array}\right]
$$

so that, $\widetilde{e}=e^{-1}, \tilde{f}=-e^{-1} f$. Then $\widetilde{e} \tilde{f}=e^{-1}\left(-e^{-1} f\right)=-e^{-2} f$ and $\widetilde{f} \widetilde{e}=$ $-e^{-1} f e^{-1}=-q e^{-2} f$. So

$$
\widetilde{e} \widetilde{f}=-e^{-2} f=q^{-1} \widetilde{f} \widetilde{e}
$$

showing

$$
\widetilde{Z} \in H_{q^{-1}}(A) .
$$

Now we assume to the contrary that $\widetilde{Z} \in H_{q}(A)$. We show that it would lead us to a contradiction. The assumption would imply that we have

$$
\widetilde{e} \widetilde{f}=q \widetilde{f} \widetilde{e} .
$$

It follows from (4.7) and (4.8)

$$
q \widetilde{f} \widetilde{e}=q^{-1} \widetilde{f} \widetilde{e} .
$$

so that we would have

$$
q^{2} \widetilde{f} \widetilde{e}=\widetilde{f} \widetilde{e} .
$$

Since $\widetilde{e}=e^{-1}$ is invertible in $A$,

$$
\left(q^{2}-1\right) \tilde{f}=0 .
$$

As the algebra $A$ is a $\mathbb{C}$-vector space and $\widetilde{f} \neq 0$, the complex number $q^{2}-1=$ 0 which is a contradiction.

Lemma 4.12. - Let $u$ and $v$ be symbols over $\mathbb{C}$. We have shown that we find a $\mathbb{C}$-Hopf algebra

$$
\mathfrak{H}_{q}=\mathbb{C}\left\langle u, u^{-1}, v\right\rangle_{\text {alg }} /(u v-q v u)
$$

as an algebra so that

$$
\begin{gathered}
u u^{-1}=u^{-1} u=1, \quad u^{-1} v=q^{-1} v u^{-1} . \\
-1358-
\end{gathered}
$$


Definition of the algebra $\mathfrak{H}_{q}$ determines the multiplication

$$
m: \mathfrak{H}_{q} \otimes_{\mathbb{C}} \mathfrak{H}_{q} \longrightarrow \mathfrak{H}_{q},
$$

the unit

$$
\eta: \mathbb{C} \longrightarrow \mathfrak{H}_{q}
$$

that is the composition of natural morphisms

$$
\mathbb{C} \longrightarrow \mathbb{C}\left\langle u, u^{-1}, v\right\rangle_{\text {alg }}
$$

and

$$
\mathbb{C}\left\langle u, u^{-1}, v\right\rangle_{\text {alg }} \longrightarrow \mathbb{C}\left\langle u, u^{-1}, v\right\rangle_{\text {alg }} /(u v-q v u)=\mathfrak{H}_{q} .
$$

The product of matrices gives the co-multiplication

$$
\Delta: \mathfrak{H}_{q} \longrightarrow \mathfrak{H}_{q} \otimes_{\mathbb{C}} \mathfrak{H}_{q},
$$

which is a $\mathbb{C}$-algebra morphism defined by

$$
\Delta(u)=u \otimes u, \quad \Delta\left(u^{-1}\right)=u^{-1} \otimes u^{-1}, \quad \Delta(v)=u \otimes v+v \otimes 1,
$$

for the generators $u, u^{-1}, v$ of the algebra $\mathfrak{H}_{q}$, the co-unit is a $\mathbb{C}$-algebra morphism

$$
\epsilon: \mathfrak{H}_{q} \longrightarrow \mathbb{C}, \quad \epsilon(u)=\epsilon\left(u^{-1}\right)=1, \epsilon(v)=0
$$

for the generators $u, u^{-1}, v$ of the algebra $\mathfrak{H}_{q}$. The antipode

$$
S: \mathfrak{H}_{q} \longrightarrow \mathfrak{H}_{q}
$$

is a $\mathbb{C}$-anti-algebra morphism given by

$$
S(u)=u^{-1}, \quad S\left(u^{-1}\right)=u, \quad S(v)=-u^{-1} v .
$$

Let us set

$$
\mathfrak{H}_{q L^{\natural}}:=\mathfrak{H}_{q} \otimes_{\mathbb{C}} L^{\natural}
$$

so that $\mathfrak{H}_{q L^{\natural}}$ is an $L^{\natural}$-Hopf algebra. We notice that for an $L^{\natural}$-algebra $A$,

$$
\begin{aligned}
H_{q}(A) & =\operatorname{Hom}_{L^{\natural} \text {-algebra }}\left(\mathfrak{H}_{q L^{\natural}}, A\right) \\
& =\left\{\left[\begin{array}{ll}
e & f \\
0 & 1
\end{array}\right] \mid e, f \in A, e f=q f e, e \text { is invertible }\right\} .
\end{aligned}
$$

Remark 4.13. - We know by general theory that the antipode $S: H \rightarrow$ $H$ that is a linear map making a few diagrams commutative, is necessarily an anti-endomorphism of the algebra $H$ so that

$$
S(a b)=S(b) S(a) \text { for all elements } a, b \in H \text { and } S(1)=1 .
$$

See Manin [12, Section 1, 2].

The Hopf algebra $\mathfrak{H}_{q}$ is a $q$-deformation of the affine algebraic group $A F_{1 \mathbb{C}}$ of affine transformations of the affine line. 
Anyhow, we notice that the quantum group appears in this very simple example showing that quantum groups are indispensable for a Galois theory of $q s i$ field extensions.

\subsection{Observations on the Galois structures of the field extension $\mathbb{C}(t) / \mathbb{C}$}

\subsubsection{Where does quantum group structure come from?}

Let us now examine that the group structure in 2.2.6 arising from the variation of initial conditions coincides with the quantum group structure defined in 4.2.1.

To see this, we have to clearly understand the initial condition of a formal series

$$
f(W, X)=\sum_{i=0}^{\infty} X^{i} a_{i}(W) \in F(\mathbb{Z}, A[[W]])[[X]]
$$

so that the coefficients $a_{i}$ 's, which are elements of $F(\mathbb{Z}, A[[W]])$, are functions on $\mathbb{Z}$ taking values in the formal power series ring $A[[W]]$. The initial condition of $f(W, X)$ is the value of the function $f(W, 0)=a_{0}(W) \in F(\mathbb{Z}, A[[W]])$ at $n=0$ which we may denote by

$$
\left.f(W, 0)\right|_{n=0} \in A[[W]] .
$$

As in Example 2.3, we set

$$
T(W, X):=\iota(t)=(t+W) Q+X \in F\left(\mathbb{Z}, L^{\natural}[[W]]\right)[[X]] .
$$

For $A \in \mathrm{ob}\left(\mathrm{NCAlg} / L^{\natural}\right)$, we take an infinitesimal deformation $\varphi \in \mathcal{N C F}_{L / k}(A)$ so that the morphism $\varphi: \mathcal{L} \rightarrow F(\mathbb{Z}, A[[W]])[[X]]$ is determined by the image

$$
\widetilde{T}(W, X):=\varphi(t) \in F(\mathbb{Z}, A[[W]])[[X]]
$$

of $t \in L \subset \mathcal{L}$, the qsi field $L$ being a sub-algebra of $\mathcal{L}$ by the universal Hopf morphism. It follows from Lemma 4.6 that there exist $e, f \in A$ such that $e f=q f e$, the elements $e-1, f$ are nilpotent and such that

$$
\varphi(t)=(e(t+W)+f) Q+X .
$$

Therefore,

$$
\widetilde{T}(W, X)=T((t(e-1)+f)+e W, X) .
$$

Since $T(W, X)$ and $\widetilde{T}(W, X)$ satisfy

$$
\widehat{\Sigma}(\mathrm{T})=q \mathrm{~T} \text { and } \widehat{\Theta}^{(1)}(\mathrm{T})=1,
$$


their difference is measured at the initial conditions. The initial condition of $T(W, X)$ is $t+W$ and that of $\widetilde{T}(W, X)$ is $e t+f+W$. Namely, the infinitesimal deformation $\varphi$ arises from the coordinate transformation

$$
t+W \longmapsto e t+f+e W
$$

or equivalently

$$
W \longmapsto t(e-1)+f+e W .
$$

We answer the question above in Observation 9.2.

\subsubsection{Quantum Galois group $\mathrm{NC} \operatorname{Inf}-\operatorname{Gal}\left(\left(\mathbb{C}(t), \sigma, \sigma^{-1}, \theta^{*}\right) / \mathbb{C}\right)$}

The Hopf algebra $\mathfrak{H}_{q}$ in 4.2.1 defines a functor

$$
\widehat{\mathfrak{H}}_{q L^{\natural}}:\left(\mathrm{NCAlg} / L^{\natural}\right) \longrightarrow(\mathrm{Set})
$$

such that

$$
\widehat{\mathfrak{H}}_{q L^{\natural}}(A)=\left\{\psi: \mathfrak{H}_{q} \otimes_{\mathbb{C}} L^{\natural} \longrightarrow A \mid \begin{array}{r}
\psi \text { is a } L^{\natural} \text {-algebra morphism such that } \\
\psi(u)-1, \psi(v) \text { are nilpotent }
\end{array}\right\}
$$

for every $A \in \mathrm{ob}\left(\mathrm{NCAlg} / L^{\natural}\right)$. In other words, $\widehat{\mathfrak{H}}_{q L^{\natural}}$ is the formal completion of the quantum group $\mathfrak{H}_{q} \otimes_{\mathbb{C}} L^{\natural}=\mathfrak{H}_{q L}$. We can summarize our results in the following form.

THEOREM 4.14. - The quantum formal group $\widehat{\mathfrak{H}}_{q L^{\natural}}$ operates on the functor $\mathcal{N C F}_{L / k}$ in such a way that there exists a functorial isomorphisms

$$
\mathcal{N C F}_{L / k} \simeq \widehat{\mathfrak{H}}_{q L} \text {. }
$$

The restriction of the functor $\mathcal{N C F}_{L / k}$ on the sub-category $\left(\mathrm{CAlg} / L^{\natural}\right)$ gives the functorial isomorphism

$$
\left.\mathcal{N C F}_{L / k}\right|_{\left(\mathrm{CAlg} / L^{\natural}\right)} \simeq \widehat{\mathbb{G}}_{m L^{\natural}} .
$$

Or equivalently,

(1) We have not only isomorphism (4.13) of functors on the category $\left(\mathrm{NCAlg} / L^{\natural}\right)$ taking values in the category (Set) of sets, but also we can identify, by this isomorphism, the co-product of the quantum formal group $\widehat{\mathfrak{H}}_{q L^{\natural}}$ arising from the multiplication of triangular matrices in 4.2.1 with composition of coordinate transformations of the initial condition in 4.3.1. For these two reasons, we say that the quantum infinitesimal Galois group of the qsi field extension $\left(\mathbb{C}(t), \sigma, \sigma^{-1}, \theta^{*}\right) / \mathbb{C}$ is the quantum formal group $\widehat{\mathfrak{H}}_{q L^{\natural}}$. Namely,

$$
\operatorname{NCinf-Gal}\left(\left(\mathbb{C}(t), \sigma, \sigma^{-1}, \theta^{*}\right) / \mathbb{C}\right) \simeq \widehat{\mathfrak{H}}_{q L^{\natural}} .
$$


(2) The commutative infinitesimal Galois group functor $\operatorname{CInf-Gal}(L / k)$ of the qsi extension $\left(\mathbb{C}(t), \sigma, \sigma^{-1}, \theta^{*}\right) / \mathbb{C}$ on the category $\left(\mathrm{Alg} / L^{\natural}\right)$ of commutative $L^{\natural}$-algebras is isomorphic to the formal group $\widehat{\mathbb{G}}_{m}$.

The operation of quantum formal group requires a precision.

Remark 4.15. - We should be careful about the operation of quantum formal group. To be more precise, for $\varphi \in \mathcal{N C F}_{L / k}(A)$ and $\psi \in \widehat{\mathfrak{H}}_{q L^{\natural}}(A)$ so that we have

$$
\varphi(t)=(e(t+W)+f) Q+X \in F(\mathbb{Z}, A[[W]])[[X]]
$$

with $e, f \in A$ and we imagine the matrix

$$
\left[\begin{array}{cc}
\psi(u) & \psi(v) \\
0 & 1
\end{array}\right] \in M_{2}(A)
$$

corresponding to $\psi$. If the sub-sets of the algebra $A,\{\psi(u), \psi(v)\}$ and $\{e, f\}$ are element-wisely commutative, the product

$$
\psi \cdot \varphi=\varpi \in \mathcal{N C F}_{L / k}(A)
$$

is defined to be

$$
\varpi(t)=(\psi(u) e(t+W)+(\psi(u) f+\psi(v)) Q+X \in F(\mathbb{Z}, A[[W]])[[X]] .
$$

\subsubsection{Non-commutative Picard-Vessiot ring}

So far we analyzed the First Example, which is a non-linear qsi equation, according to general principle of Hopf Galois theory. We finally arrived at Theorem 4.14 that shows a quantum formal group appears as a Galois group. Our experiences of dealing Picard-Vessiot theory in our general framework done in our previous works $[22,26]$, teach us that we discovered here a new phenomenon, a non-commutative Picard-Vessiot extension.

We work in the qsi ring $\left(F(\mathbb{Z}, \mathbb{C}(t))[[X]], \widehat{\Sigma}, \widehat{\Sigma}^{-1}, \widehat{\Theta}^{*}\right)$. We are delighted to assert that a non-commutative qsi ring extension

$$
\left.\left(\mathbb{C}\left\langle Q, Q^{-1}, X\right\rangle_{\text {alg }}, \widehat{\Sigma}, \widehat{\Sigma}^{-1}, \widehat{\Theta}^{*}\right) / \mathbb{C}\right)
$$

is a non-commutative Picard Vessiot ring with quantum Galois group $\mathfrak{H}_{q}$. We consider the fundamental system

$$
Y:=\left[\begin{array}{cc}
Q & X \\
0 & 1
\end{array}\right] \in M_{2}\left(\mathbb{C}\left\langle Q, Q^{-1}, X\right\rangle_{\mathrm{alg}}\right)
$$

so that the homogeneous linear qsi equations are

$$
\widehat{\Sigma}(Y)=\left[\begin{array}{ll}
q & 0 \\
0 & 1
\end{array}\right] Y, \quad \widehat{\Theta}^{(1)}(Y)=\left[\begin{array}{ll}
0 & 1 \\
0 & 0
\end{array}\right] Y .
$$


In fact, we can check the first equation in (4.15):

$$
\widehat{\Sigma}(Y)=\left[\begin{array}{cc}
\widehat{\Sigma}(Q) & \widehat{\Sigma}(X) \\
\widehat{\Sigma}(0) & \widehat{\Sigma}(1)
\end{array}\right]=\left[\begin{array}{cc}
q Q & q X \\
0 & 1
\end{array}\right]=\left[\begin{array}{cc}
q & 0 \\
0 & 1
\end{array}\right]\left[\begin{array}{cc}
Q & X \\
0 & 1
\end{array}\right]=\left[\begin{array}{cc}
q & 0 \\
0 & 1
\end{array}\right] Y .
$$

The second equality of (4.15) is also checked easily.

Leaving heuristic reasoning totally aside, we study the Picard-Vessiot extension (4.14) in detail in Sections 9 and 10.

\section{The Second Example, the qsi field extension$$
L / k=\left(\mathbb{C}\left(t, t^{\alpha}\right), \sigma, \sigma^{-1}, \theta^{*}\right) / \mathbb{C}
$$

\subsection{Commutative deformations}

As in the previous Section, let $t$ be a variable over $\mathbb{C}$ and we assume that the complex number $q$ is not a root of unity if we do not mention other assumptions on $q$. Sometimes we write the condition that $q$ is not a root of unity, simply to recall it. We work under the condition that $\alpha$ is an irrational complex number so that $t$ and $t^{\alpha}$ are algebraically independent over $\mathbb{C}$. Therefore, the field $\mathbb{C}\left(t, t^{\alpha}\right)$ is isomorphic to the rational function field of two variables over $\mathbb{C}$. Let $q^{\alpha}=\exp (\alpha \log q)$, choosing arbitrarily a branch of the logarithm. Let $\sigma$ denote the $\mathbb{C}$-automorphism of the field $\mathbb{C}\left(t, t^{\alpha}\right)$ such that

$$
\sigma(t)=q t \quad \text { and } \quad \sigma\left(t^{\alpha}\right)=q^{\alpha} t^{\alpha} .
$$

Let us set $\theta^{(0)}:=\operatorname{Id}_{\mathbb{C}\left(t, t^{\alpha}\right)}$, the map

$$
\theta^{(1)}:=\frac{\sigma-\operatorname{Id}_{\mathbb{C}\left(t, t^{\alpha}\right)}}{(q-1) t}: \mathbb{C}\left(t, t^{\alpha}\right) \longrightarrow \mathbb{C}\left(t, t^{\alpha}\right)
$$

and

$$
\theta^{(n)}=\frac{1}{[n]_{q} !}\left(\theta^{(1)}\right)^{n} \quad \text { for } n=2,3, \ldots
$$

So the $\theta^{(i)}$ 's are $\mathbb{C}$-linear operators on $\mathbb{C}\left(t, t^{\alpha}\right)$ and

$$
L:=\left(\mathbb{C}\left(t, t^{\alpha}\right), \sigma, \sigma^{-1}, \theta^{*}\right)
$$

is a $q s i$ field. The restriction of $\sigma$ and $\theta^{*}$ to the subfield $\mathbb{C}$ are trivial. We denote the $q s i$ field extension $L / \mathbb{C}$ by $L / k$. We denote $t^{\alpha}$ by $y$ so that as we mentioned above, the abstract field $\mathbb{C}\left(t, t^{\alpha}\right)=\mathbb{C}(t, y)$ is isomorphic to the rational function field of two variables over $\mathbb{C}$. We take the derivations $\partial / \partial t$ and $\partial / \partial y$ as a basis of the $L^{\natural}$-vector space $\operatorname{Der}\left(L^{\natural} / k^{\natural}\right)$ of $k^{\natural}$-derivations of $L^{\natural}$. Hence $L^{\sharp}=\left(L^{\natural},\{\partial / \partial t, \partial / \partial y\}\right)$ as in [26]. 
Let us list the fundamental equations.

$$
\begin{aligned}
\sigma(t) & =q t, & \sigma(y) & =q^{\alpha} y, \\
\theta^{(1)}(t) & =1, & \theta^{(1)}(y) & =[\alpha]_{q} \frac{y}{t} .
\end{aligned}
$$

We explain below the notation $[\alpha]_{q}$. We are going to determine the Galois group

$$
\operatorname{NC} \operatorname{Inf}-\operatorname{Gal}(L / k) \text {. }
$$

Before we start, we notice that by Proposition 4.5, the Galois hull of the extension $\left(\mathbb{C}(t), \sigma, \sigma^{-1}, \theta^{*}\right) / \mathbb{C}$ is not a commutative algebra and since $\mathbb{C}(t)$ is a sub-field of $\mathbb{C}\left(t, t^{\alpha}\right)$, the Galois hull of the qsi field extension $\left(\mathbb{C}\left(t, t^{\alpha}\right), \sigma, \sigma^{-1}, \theta^{*}\right) / \mathbb{C}$ is not a commutative algebra either. Consequently the qsi field extension $\left(\mathbb{C}\left(t, t^{\alpha}\right), \sigma, \sigma^{-1}, \theta^{*}\right) / \mathbb{C}$ is not a Picard-Vessiot extension. See [5], [14] and [26]. So we have to go beyond the general theory of Heiderich [7], Umemura [26] for the definition of the Galois group $\operatorname{NC} \operatorname{Inf}-G a l(L / k)$.

It follows from general definition that the universal Hopf morphism

$$
\iota: L \rightarrow F\left(\mathbb{Z}, L^{\natural}\right)[[X]]
$$

is given by

$$
\iota(a)=\sum_{n=0}^{\infty} X^{n} u\left[\theta^{(n)}(a)\right] \in F\left(\mathbb{Z}, L^{\natural}\right)[[X]]
$$

for $a \in L$. Here for $b \in L$, we denote by $u[b]$ the element

$$
u[b]=\left[\begin{array}{ccccccc}
\cdots & -2 & -1 & 0 & 1 & 2 & \cdots \\
\cdots & \sigma^{-2}(b) & \sigma^{-1}(b) & b & \sigma(b) & \sigma^{2}(b) & \cdots
\end{array}\right] \in F\left(\mathbb{Z}, L^{\natural}\right) .
$$

It follows from the definition above of the universal Hopf morphism $\iota$,

$$
\iota(y)=\sum_{n=0}^{\infty} X^{n}\left(\begin{array}{l}
\alpha \\
n
\end{array}\right)_{q} t^{-n} Q^{\alpha-n} y,
$$

where we use the following notations. For a complex number $\beta \in \alpha+\mathbb{Z}$,

$$
[\beta]_{q}=\frac{q^{\beta}-1}{q-1}
$$

and

$$
\begin{aligned}
\left(\begin{array}{l}
\alpha \\
n
\end{array}\right)_{q} & =\frac{[\alpha]_{q}[\alpha-1]_{q} \ldots[\alpha-n+1]_{q}}{[n]_{q} !} \\
Q & =\left[\begin{array}{ccccccc}
\ldots & -2 & -1 & 0 & 1 & 2 & \ldots \\
\cdots & q^{-2} & q^{-1} & 1 & q & q^{2} & \ldots
\end{array}\right] \\
\text { and } \quad Q^{\alpha} & =\left[\begin{array}{ccccccc}
\cdots & -2 & -1 & 0 & 1 & 2 & \ldots \\
\cdots & q^{-2 \alpha} & q^{-\alpha} & 1 & q^{\alpha} & q^{2 \alpha} & \ldots
\end{array}\right] .
\end{aligned}
$$


We set

$$
Y_{0}:=\sum_{n=0}^{\infty} X^{n}\left(\begin{array}{l}
\alpha \\
n
\end{array}\right)_{q} t^{-n} Q^{\alpha-n}
$$

so that

$$
\iota(y)=Y_{0} y \quad \text { in } F\left(\mathbb{Z}, L^{\natural}\right)[[X]] .
$$

Considering $k^{\natural}$-derivations $\partial / \partial t, \partial / \partial y$ in $L^{\natural}$ and therefore in $F\left(\mathbb{Z}, L^{\natural}\right)$ or in $F\left(\mathbb{Z}, L^{\natural}\right)[[X]]$, we generate the Galois hull $\mathcal{L}$ by $\iota(L)$ and $L^{\natural}$ so that $\mathcal{L} \subset$ $F\left(\mathbb{Z}, L^{\natural}\right)[[X]]$ is invariant under $\widehat{\Sigma}$, the $\widehat{\Theta}^{(i)}$ 's and $\{\partial / \partial t, \partial / \partial y\}$. We may thus consider

$$
\mathcal{L} \longleftrightarrow F\left(\mathbb{Z}, L^{\sharp}\right)[[X]] .
$$

By the universal Taylor morphism

$$
L^{\sharp}=\left(L^{\natural},\{\partial / \partial t, \partial / \partial y\}\right) \longrightarrow L^{\sharp}\left[\left[W_{1}, W_{2}\right]\right],
$$

we identify $\mathcal{L}$ by the canonical morphism

$$
\iota: \mathcal{L} \longrightarrow F\left(\mathbb{Z}, L^{\sharp}\right)[[X]] \longrightarrow F\left(\mathbb{Z}, L^{\natural}\left[\left[W_{1}, W_{2}\right]\right]\right)[[X]] .
$$

We study first the infinitesimal deformations $\mathcal{C F}_{L / k}$ of $\iota$ on the category $\left(C A l g / L^{\natural}\right)$ of commutative $L^{\natural}$-algebras and then generalize the argument to the category (NCAlg $/ L^{\natural}$ ) of not necessarily commutative $L^{\natural}$-algebras.

For a commutative $L^{\natural}$-algebra $A$, let $\varphi: \mathcal{L} \rightarrow F\left(\mathbb{Z}, A\left[\left[W_{1}, W_{2}\right]\right]\right)[[X]]$ be an infinitesimal deformation of the canonical morphism $\iota: \mathcal{L} \rightarrow$ $F\left(\mathbb{Z}, L^{\natural}\left[\left[W_{1}, W_{2}\right]\right]\right)[[X]]$ so that both $\iota$ and $\varphi$ are compatible with operators $\left\{\widehat{\Sigma}, \widehat{\Theta}^{*}, \partial / \partial W_{1}, \partial / \partial W_{2}\right\}$.

LEMmA 5.1. - The infinitesimal deformation $\varphi$ is determined by the images $\varphi\left(Y_{0}\right), \varphi(Q)$ and $\varphi(X)$.

Proof. - The Galois hull $\mathcal{L} / \mathcal{K}$ is generated over $\mathcal{K}=L^{\sharp}$ by $\iota(t)=t Q+X$ and $\iota(y)=Y_{0} y$ with operators $\widehat{\Theta}^{*}, \widehat{\Sigma}$ and $\partial / \partial t, \partial / \partial y$ along with localizations. This proves the Lemma.

Let us set $Z_{0}:=\varphi\left(Y_{0}\right) \in F\left(\mathbb{Z}, A\left[\left[W_{1}, W_{2}\right]\right]\right)[[X]]$ and expand it into a formal power series in $X$ :

$$
Z_{0}=\sum_{n=0}^{\infty} X^{n} a_{n}, \text { with } a_{n} \in F\left(\mathbb{Z}, A\left[\left[W_{1}, W_{2}\right]\right]\right) \quad \text { for every } n \in \mathbb{N} .
$$

It follows from (5.1) and (5.3)

$$
\widehat{\Sigma}\left(Z_{0}\right)=q^{\alpha} Z_{0}
$$

so that

$$
\sum_{n=0}^{\infty} X^{n} q^{n} \widehat{\Sigma}\left(a_{n}\right)=q^{\alpha} \sum_{n=0}^{\infty} X^{n} a_{n}
$$


Comparing the coefficient of the $X^{n}$ 's in (5.4) we get

$$
\widehat{\Sigma}\left(a_{n}\right)=q^{\alpha-n} a_{n} \quad \text { for } n \in \mathbb{N} .
$$

So $a_{n}=b_{n} Q^{\alpha-n}$ with $b_{n} \in A\left[\left[W_{1}, W_{2}\right]\right]$ for $n \in \mathbb{N}$. Namely we have

$$
Z_{0}=\sum_{n=0}^{\infty} X^{n} b_{n} Q^{\alpha-n} \quad \text { with } b_{n} \in A\left[\left[W_{1}, W_{2}\right]\right] .
$$

It follows from (5.2),

$$
\sigma(y)-y=\theta^{(1)}(y)(q-1) t
$$

and so by (5.1)

$$
\left(q^{\alpha}-1\right) y=\theta^{(1)}(y)(q-1) t .
$$

Applying the canonical morphism $\iota$ and the deformation $\varphi$, we get

$$
\left(q^{\alpha}-1\right) Y_{0}=\widehat{\Theta}^{(1)}\left(Y_{0}\right)(q-1)(t Q+X)
$$

as well as by the argument of First Example,

$$
\left(q^{\alpha}-1\right) Z_{0}=\widehat{\Theta}^{(1)}\left(Z_{0}\right)(q-1)(t e Q+X)
$$

where $e \in A$ is an invertible element congruent to 1 modulo nilpotent elements.

Substituting (5.5) into (5.7), we get a recurrence relation among the $b_{m}$ 's;

$$
b_{m+1}=\frac{[\alpha-m]_{q}}{[m+1]_{q}\left(e\left(t+W_{1}\right)\right)} b_{m} .
$$

Hence

$$
b_{m}=\left(\begin{array}{c}
\alpha \\
m
\end{array}\right)_{q}\left(e\left(t+W_{1}\right)\right)^{-m} b_{0} \quad \text { for every } m \in \mathbb{N},
$$

where $b_{0} \in A\left[\left[W_{1}, W_{2}\right]\right]$ and every coefficient of the power series $b_{0}-1$ are nilpotent.

Since

$$
\frac{\partial Y_{0}}{\partial y}=\frac{\partial}{\partial W_{2}}\left(\sum_{n=0}^{\infty} X^{n}\left(\begin{array}{l}
\alpha \\
n
\end{array}\right)_{q}\left(t+W_{1}\right)^{-n} Q^{\alpha-n}\right)=0
$$

we must have

$$
0=\varphi\left(\frac{\partial Y_{0}}{\partial y}\right)=\frac{\partial \varphi\left(Y_{0}\right)}{\partial W_{2}}=\frac{\partial Z_{0}}{\partial W_{2}}
$$

and consequently

$$
\frac{\partial b_{0}}{\partial W_{2}}=0
$$

so that

$$
\begin{aligned}
b_{0} & \in A\left[\left[W_{1}\right]\right] . \\
& -1366-
\end{aligned}
$$


by (5.5). Therefore, we have determined the image

$$
Z_{0}=\varphi\left(Y_{0}\right)=\sum_{n=0}^{\infty} X^{n}\left(\begin{array}{l}
\alpha \\
n
\end{array}\right)_{q}\left(e\left(t+W_{1}\right)\right)^{-n} Q^{\alpha-n} b_{0}
$$

by (5.8), where all the coefficients of the power series $b_{0}\left(W_{1}\right)-1$ are nilpotent.

\subsection{Commutative deformation functor $\mathcal{C F}_{L / k}$ for $\mathbb{C}\left(t, t^{\alpha}\right) / \mathbb{C}$}

In the Second Example, when we deal with the qsi field extension $L / k$, the Galois hull $\mathcal{L} / \mathcal{K}$ is a non-commutative algebra extension. So we have to consider the functor $\mathcal{N C F}_{L / k}$ on the category (NCAlg $/ L^{\natural}$ ) of not necessarily commutative $L^{\natural}$-algebras. It is, however, easier to understand first the commutative deformation functor $\mathcal{C F}_{L / k}$ that is the restriction on the sub-category (CAlg / $L^{\natural}$ ) of the functor $\mathcal{N C F}_{L / k}$. We using the notation of Lemma 5.1, it follows from (5.9) the following Proposition.

Proposition 5.2. - We set

$$
\begin{aligned}
Y_{1}\left(W_{1}, W_{2} ; X\right) & :=\left(t+W_{1}\right) Q+X \\
Y_{2}\left(W_{1}, W_{2} ; X\right) & :=\sum_{n=0}^{\infty} X^{n}\left(\begin{array}{l}
\alpha \\
n
\end{array}\right)_{q}\left(t+W_{1}\right)^{-n} Q^{\alpha-n}\left(y+W_{2}\right) .
\end{aligned}
$$

Then we have

$$
\begin{aligned}
& \iota(t)=Y_{1}\left(W_{1}, W_{2} ; X\right), \\
& \iota(y)=Y_{2}\left(W_{1}, W_{2} ; X\right)
\end{aligned}
$$

and

$$
\begin{aligned}
& \varphi\left(Y_{1}\left(W_{1}, W_{2} ; X\right)\right):=Y_{1}\left((e-1) t+e W_{1},\left[b_{0}\left(W_{1}\right)-1\right] y+b_{0}\left(W_{1}\right) W_{2} ; X\right), \\
& \varphi\left(Y_{2}\left(W_{1}, W_{2} ; X\right)\right):=Y_{2}\left((e-1) t+e W_{1},\left[b_{0}\left(W_{1}\right)-1\right] y+b_{0}\left(W_{1}\right) W_{2} ; X\right) .
\end{aligned}
$$

In other words, the infinitesimal deformation $\varphi$ is given by the coordinate transformation of the initial conditions

$$
\left(W_{1}, W_{2}\right) \longmapsto\left(\varphi_{1}\left(W_{1}, W_{2}\right), \varphi_{2}\left(W_{1}, W_{2}\right)\right),
$$

where

$$
\begin{aligned}
& \varphi_{1}\left(W_{1}, W_{2}\right)=(e-1) t+e W_{1}, \\
& \varphi_{2}\left(W_{1}, W_{2}\right)=\left[b_{0}\left(W_{1}\right)-1\right] y+b_{0}\left(W_{1}\right) W_{2} .
\end{aligned}
$$

The set of transformations in the form of (5.16), (5.17) forms a group. 
Lemma 5.3. - For a commutative $L^{\natural}$-algebra $A$, we set

$$
\begin{aligned}
& \widehat{G}_{I I}(A) \\
& :=\left\{\begin{array}{l|l}
\left((e-1) t+e W_{1},\left[b\left(W_{1}\right)-1\right] y+b\left(W_{1}\right) W_{2}\right) & \begin{array}{l}
e \in A, b\left(W_{1}\right) \in A\left[\left[W_{1}\right]\right], \\
\text { all the coefficients of } b\left(W_{1}\right)-1 \\
\text { and } e-1 \text { are nilpotent }
\end{array}
\end{array}\right\} .
\end{aligned}
$$

Then the set $\widehat{G}_{I I}(A)$ is a group, the group law being the composition of coordinate transformations.

Proof. - We have shown in Umemura [22] that the set of coordinate transformations of $n$-variables with coefficients in a commutative ring that are congruent to the identity modulo nilpotent elements forms a group under the composite of transformations. So it is sufficient to show:

(1) The set $\widehat{G}_{I I}(A)$ is closed under the composition.

(2) The identity is in $\widehat{G}_{I I}(A)$.

(3) The inverse of every element in $\widehat{G}_{I I}(A)$ is in $\widehat{G}_{I I}(A)$.

In fact, let

$$
\begin{aligned}
& \left((e-1) t+e W_{1},\left[b\left(W_{1}\right)-1\right] y+b\left(W_{1}\right) W_{2}\right), \\
& \left((f-1) t+f W_{1},\left[c\left(W_{1}\right)-1\right] y+c\left(W_{1}\right) W_{2}\right)
\end{aligned}
$$

be two elements of $\widehat{G}_{I I}(A)$. We mean by their composite

$$
\begin{aligned}
& \left((e f-1) t+e f W_{1},\right. \\
& \left.\quad\left[b\left((f-1) t+f W_{1}\right) c\left(W_{1}\right)-1\right] y+b\left((f-1) t+f W_{1}\right) c\left(W_{1}\right) W_{2}\right)
\end{aligned}
$$

that is an element of $\widehat{G}_{I I}(A)$. Certainly the identity $\left(W_{1}, W_{2}\right)$ is expressed for $e=1$ and $b\left(W_{1}\right)=1$. As for the inverse

$$
\begin{aligned}
\left((e-1) t+e W_{1},\left[b\left(W_{1}\right)-1\right] y+b\left(W_{1}\right) W_{2}\right)^{-1} \\
\quad=\left(\left(e^{-1}-1\right) t+e^{-1} W_{1},\left[c\left(W_{1}\right)-1\right] y+c\left(W_{1}\right) W_{2}\right),
\end{aligned}
$$

where

$$
c\left(W_{1}\right)=\frac{1}{b\left(\left(e^{-1}-1\right) t+e^{-1} W_{1}\right)} .
$$

We can summarize what we have proved as follows.

Proposition 5.4. - There exists a functorial inclusion on the category (CAlg $\left./ L^{\natural}\right)$ of commutative $L^{\natural}$-algebras

$$
\mathcal{C F}_{L / k}(A):=\left.\mathcal{N C F}_{L / k}\right|_{\left(\mathrm{CAlg} / L^{\natural}\right)}(A) \hookrightarrow \widehat{G}_{I I}(A)
$$

that sends infinitesimal deformation $\varphi$ to

$$
\left((e-1) t+e W_{1},\left[b_{0}\left(W_{1}\right)-1\right] y+b_{0}\left(W_{1}\right) W_{2}\right) \in \widehat{G}_{I I}(A)
$$


for every commutative $L^{\natural}$-algebra $A$.

In the Definition of the group functor $\widehat{G}_{I I}$ in Lemma 5.3, we can eliminate the variable $W_{2}$.

Lemma 5.5. - We introduce a group functor

$$
\widehat{G}_{2}:\left(\mathrm{CAlg} / L^{\natural}\right) \longrightarrow(\mathrm{Grp}),
$$

setting

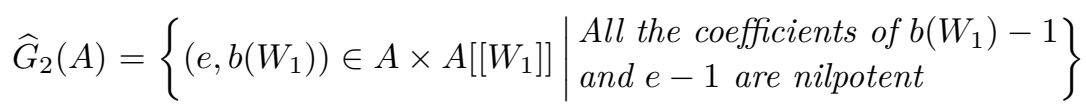

for every $A \in \mathrm{ob}\left(\mathrm{CAlg} / L^{\natural}\right)$. The group law, the identity and the inverse are given as below.

For two elements $\left(e, b\left(W_{1}\right)\right),\left(f, c\left(W_{1}\right)\right)$, their product is by definition

$$
\left(e f, b\left((f-1) t+f W_{1}\right) c\left(W_{1}\right)\right) .
$$

The identity is $(1,1)$ and the inverse

$$
\left(e, b\left(W_{1}\right)\right)^{-1}=\left(\frac{1}{e}, \frac{1}{b\left(\left(e^{-1}-1\right) t+e^{-1} W_{1}\right)}\right) .
$$

Then there exists an isomorphism of group functors.

$$
\widehat{G}_{I I} \simeq \widehat{G}_{2} .
$$

Proof. - In fact, for a every commutative algebra $A \in \mathrm{ob}\left(\mathrm{CAlg} / L^{\natural}\right)$, the map

$$
\begin{aligned}
\widehat{G}_{I I}(A) & \longrightarrow \widehat{G}_{2}(A), \\
\left((e-1) t+e W_{1},\left(b\left(W_{1}\right)-1\right) y+b\left(W_{1}\right) W_{2}\right) & \longmapsto\left(e, b\left(W_{1}\right)\right)
\end{aligned}
$$

gives an isomorphism of group functors.

Remark 5.6. - In the composition laws for $\widehat{G}_{I I}$ (5.19) and for $\widehat{G}_{2}(5.20)$, we substitute in the variable $W_{1}$ the linear polynomial $(e-1) t+W_{1}$ in the power series $c\left(W_{1}\right)$ to get $c\left((e-1) t+e W_{1}\right)$. Since $c\left(W_{1}\right)$ is a power series, in order that the substitution has sense, we can not avoid the condition that $e-1$ is nilpotent. We can neither define the global group functors $G_{I I}$ nor $G_{2}$ whose completions are $\widehat{G}_{I I}, \widehat{G}_{2}$ respectively.

It is natural to wonder what is the image of the inclusion map in Proposition 5.4.

Conjecture 5.7. - If $q$ is not a root of unity, the inclusion in Proposition 5.4 is the equality. 
Proposition 5.8. - Origin of the group structure teaches us that if the Conjecture 5.7 is true, then the group functor

$$
\widehat{G}_{I I}:\left(\mathrm{CAlg} / L^{\natural}\right) \longrightarrow(\mathrm{Grp})
$$

operates on the functor

$$
\mathcal{C F}_{L / k}:\left(\mathrm{CAlg} / L^{\natural}\right) \longrightarrow(\mathrm{Set})
$$

through the transformations of the initial conditions $\left(W_{1}, W_{2}\right)$, in such a way that

$$
\left(\widehat{G}_{I I}, \mathcal{C F} \mathcal{F}_{L / k}\right)
$$

is a torsor. So we may say that the Galois group functor

$$
\operatorname{CInf-Gal}\left(\left(\mathbb{C}\left(t, t^{\alpha}\right) \sigma, \sigma^{-1}, \theta^{*}\right) / \mathbb{C}\right) \simeq \widehat{G}_{I I}
$$

Remark 5.9. - We explain a background of Conjecture 5.7.

LEMMA 5.10. - The Galois hull $\mathcal{L}$ is a localization of the following ring

$$
L^{\sharp}\left\langle Q, X, \frac{1}{t Q+X}\right\rangle_{\text {alg }}\left\langle\frac{\partial^{l}}{\partial t^{l}} Y_{0}\right\rangle_{a l g, l \in \mathbb{N}} .
$$

Proof. - Since $\iota(t)=t Q+X$, as we have seen in the First Example,

$$
L^{\sharp}\langle Q, X\rangle_{\text {alg }}\left\langle\frac{\partial^{l}}{\partial t^{l}} Y_{0}\right\rangle_{a l g, l \in \mathbb{N}} \subset \mathcal{L} .
$$

We show that the ring

$$
L^{\sharp}\langle Q, X\rangle_{\text {alg }}\left\langle\frac{\partial^{l}}{\partial t^{l}} Y_{0}\right\rangle_{a l g, l \in \mathbb{N}}
$$

is closed under the operations $\widehat{\Sigma}, \widehat{\Theta}^{(i)}, \partial / \partial t$ and $\partial / \partial y$ of $F\left(\mathbb{Z}, L^{\sharp}\right)[[X]]$. Evidently the ring is closed under the last two operators. Since the operators $\widehat{\Sigma}$ and $\partial^{n} / \partial t^{n}$ operating on $F\left(\mathbb{Z}, L^{\sharp}\right)[[X]]$ mutually commute, it follows from (5.1)

$$
\widehat{\Sigma}\left(\frac{\partial^{n} Y_{0}}{\partial t^{n}}\right)=\frac{\partial^{n}}{\partial t^{n}} \widehat{\Sigma}\left(Y_{0}\right)=\frac{\partial^{n}}{\partial t^{n}}\left(q^{\alpha} Y_{0}\right)=q^{\alpha} \frac{\partial^{n} Y_{0}}{\partial t^{n}}
$$


So the ring is closed under $\widehat{\Sigma}$. Similarly since the operators $\widehat{\Theta}^{(1)}$ and $\partial^{n} / \partial t^{n}$ mutually commute on $F\left(\mathbb{Z}, L^{\sharp}\right)[[X]]$,

$$
\begin{aligned}
\widehat{\Theta}^{(1)}\left(\frac{\partial^{n} Y_{0}}{\partial t^{n}}\right) & =\frac{\partial^{n}}{\partial t^{n}} \widehat{\Theta}^{(1)}\left(Y_{0}\right) \\
& =\frac{1}{y} \frac{\partial^{n}}{\partial t^{n}} \widehat{\Theta}^{(1)}\left(Y_{0} y\right) \\
& =\frac{1}{y} \frac{\partial^{n}}{\partial t^{n}} \widehat{\Theta}^{(1)}(\iota(y)) \\
& =\frac{1}{y} \frac{\partial^{n}}{\partial t^{n}} \iota\left(\theta^{(1)}(y)\right) \\
& =\frac{1}{y} \frac{\partial^{n}}{\partial t^{n}} \iota\left(\frac{\sigma(y)-y}{(q-1) t}\right) \\
& =\frac{1}{y} \frac{\partial^{n}}{\partial t^{n}}\left(\frac{q^{\alpha} Y_{0} y-Y_{0} y}{(q-1)(t Q+X)}\right) \\
& =\frac{1}{y} \frac{\partial^{n}}{\partial t^{n}}\left(\frac{q^{\alpha} Y_{0}-Y_{0}}{(q-1)(t Q+X)}\right),
\end{aligned}
$$

which is an element of the ring.

Conjecture 5.7 arises from experience that if $q$ is not a root of unity, we could not find any non-trivial algebraic relations among the partial derivatives

$$
\frac{\partial^{n} Y_{0}}{\partial t^{n}} \quad \text { for } n \in \mathbb{N}
$$

over $L^{\sharp}$ so that we could guess that there would be none.

In fact, assume that we could prove our guess. Let $\varphi: \mathcal{L} \rightarrow F(\mathbb{Z}$, $\left.A\left[\left[W_{1}, W_{2}\right]\right]\right)[[X]]$ be an infinitesimal deformation of $\iota$. So as we have seen

$$
Z_{0}=\varphi\left(Y_{0}\right)=\sum_{n=0}^{\infty} X^{n}\left(\begin{array}{l}
\alpha \\
n
\end{array}\right)_{q}\left(e\left(t+W_{1}\right)\right)^{-n} Q^{\alpha-n} b\left(W_{1}\right)
$$

with $b\left(W_{1}\right) \in A\left[\left[W_{1}\right]\right]$. There would be no constraints among the partial derivatives $\partial^{n} b\left(W_{1}\right) / \partial W_{1}^{n}, n \in \mathbb{N}$ and hence we could choose any power series $b\left(W_{1}\right) \in A\left[\left[W_{1}\right]\right]$ such that every coefficient of the power series $b\left(W_{1}\right)-1$ is nilpotent.

\subsection{Non-commutative deformation functor $\mathcal{N C F}_{L / k}$ for $\mathbb{C}\left(t, t^{\alpha}\right) / \mathbb{C}$}

We study the functor $\mathcal{N C F}_{L / k}(A)$ of non-commutative deformations

$$
\mathcal{N C F}_{L / k}:\left(\mathrm{NCAlg} / L^{\natural}\right) \longrightarrow(\mathrm{Set})
$$


For a not necessarily commutative $L^{\natural}$-algebra $A \in o b\left(\mathrm{NCAlg} / L^{\natural}\right)$, let

$$
\varphi: \mathcal{L} \longrightarrow F\left(\mathbb{Z}, A\left[\left[W_{1}, W_{2}\right]\right]\right)[[X]]
$$

be an infinitesimal deformation of the canonical morphism

$$
\iota: \mathcal{L} \longrightarrow F\left(\mathbb{Z}, A\left[\left[W_{1}, W_{2}\right]\right]\right)[[X]] .
$$

Both $t$ and $y$ are elements of the field $\mathbb{C}\left(t, t^{\alpha}\right)=\mathbb{C}(t, y)$ so that $[t, y]=$ $t y-y t=0$. So for the deformation $\varphi \in \mathcal{N C F}_{L / k}(A)$ we must have

$$
[\varphi(t), \varphi(y)]=\varphi(t) \varphi(y)-\varphi(y) \varphi(t)=0 .
$$

When we consider the non-commutative deformations, the commutativity (5.24) gives a constraint for the deformation. To see this, we need a Lemma.

Lemma 5.11. - For every $l \in \mathbb{N}$, we have

$$
q^{l}\left(\begin{array}{c}
\alpha \\
l
\end{array}\right)_{q}+\left(\begin{array}{c}
\alpha \\
l-1
\end{array}\right)_{q}=\left(\begin{array}{l}
\alpha \\
l
\end{array}\right)_{q}+q^{\alpha-l+1}\left(\begin{array}{c}
\alpha \\
l-1
\end{array}\right)_{q} .
$$

Proof. - This follows from the definition of $q$-binomial coefficient.

Lemma 5.12. - Let $A$ be a not necessarily commutative $L^{\natural}$-algebra in $\mathrm{ob}\left(\mathrm{NCAlg} / L^{\natural}\right)$. Let $e, f \in A$ such that $e-1$ and $f$ are nilpotent. We set

$$
\mathcal{A}:=\left(e\left(t+W_{1}\right)+f\right) Q+X
$$

and for a power series $b\left(W_{1}\right) \in A\left[\left[W_{1}\right]\right]$, we also set

$$
\mathcal{Z}:=\sum_{n=0}^{\infty} X^{n}\left(\begin{array}{l}
\alpha \\
n
\end{array}\right)_{q}\left(e\left(t+W_{1}\right)+f\right)^{-n} Q^{\alpha-n} b\left(W_{1}\right)
$$

so that $\mathcal{A}$ and $\mathcal{Z}$ are elements of $F\left(\mathbb{Z}, A\left[\left[W_{1}\right]\right]\right)[[X]]$. The following conditions are equivalent.

(1) $[\mathcal{A}, \mathcal{Z}]:=\mathcal{A Z}-\mathcal{Z} \mathcal{A}=0$.

(2) $\left[e\left(t+W_{1}\right)+f, b\left(W_{1}\right)\right]=0$.

Proof. - We formulate condition (1) in terms of coefficients of the power series in $X$. Assume condition (1) holds so that we have

$$
\begin{aligned}
& \left(\left(e\left(t+W_{1}\right)+f\right) Q+X\right)\left(\sum_{n=0}^{\infty} X^{n}\left(\begin{array}{l}
\alpha \\
n
\end{array}\right)_{q}\left(e\left(f+W_{1}\right)+f\right)^{-n} Q^{\alpha-n} b\left(W_{1}\right)\right) \\
& =\left(\sum_{n=0}^{\infty} X^{n}\left(\begin{array}{l}
\alpha \\
n
\end{array}\right)_{q}\left(e\left(f+W_{1}\right)+f\right)^{-n} Q^{\alpha-n} b\left(W_{1}\right)\right)\left(\left(e\left(t+W_{1}\right)+f\right) Q+X\right) .
\end{aligned}
$$


Comparing degree $l$ terms in $X$ of (5.25), we find that condition (1) is equivalent to

$$
\begin{aligned}
& q^{l}\left(\begin{array}{c}
\alpha \\
l
\end{array}\right)_{q}(e(t+\left.\left.W_{1}\right)+f\right)^{-l+1} Q^{\alpha-l+1} b\left(W_{1}\right) \\
&+\left(\begin{array}{c}
\alpha \\
l-1
\end{array}\right)_{q}\left(e\left(t+W_{1}\right)+f\right)^{-l+1} Q^{\alpha-l+1} b\left(W_{1}\right) \\
&=\left(\begin{array}{c}
\alpha \\
l
\end{array}\right)_{q}\left(e\left(t+W_{1}\right)+f\right)^{-l} b\left(W_{1}\right)\left(e\left(t+W_{1}\right)+f\right) Q^{\alpha-l+1} \\
&+\left(\begin{array}{c}
\alpha \\
l-1
\end{array}\right)_{q} q^{\alpha-l+1}\left(e\left(t+W_{1}\right)+f\right)^{-l+1} Q^{\alpha-l+1} b\left(W_{1}\right) .
\end{aligned}
$$

So the condition (1) is equivalent to

$$
\begin{aligned}
& q^{l}\left(\begin{array}{c}
\alpha \\
l
\end{array}\right)_{q}\left(e\left(t+W_{1}\right)+f\right) b\left(W_{1}\right)+\left(\begin{array}{c}
\alpha \\
l-1
\end{array}\right)_{q}\left(e\left(t+W_{1}\right)+f\right) b\left(W_{1}\right) \\
& =\left(\begin{array}{c}
\alpha \\
l
\end{array}\right)_{q} b\left(W_{1}\right)\left(e\left(t+W_{1}\right)+f\right)+\left(\begin{array}{c}
\alpha \\
l-1
\end{array}\right)_{q} q^{\alpha-l+1}\left(e\left(t+W_{1}\right)+f\right) b\left(W_{1}\right)
\end{aligned}
$$

for every $l \in \mathbb{N}$. Condition (5.27) for $l=0$ is condition (2). Hence condition (1) implies condition (2). Conversely condition (1) follows from (2) in view of (5.27) and Lemma 5.11.

Now let us come back to the infinitesimal deformation (5.23) of the canonical morphism $\iota$. The argument in Section 4 allows us to determine the restriction $\varphi$ on the sub-algebra generated by $\iota(t)=t Q+X$ over $L^{\sharp}$ invariant under the $\widehat{\Theta}^{(i)}$ 's, $\widehat{\Sigma}$ and $\{\partial / \partial t, \partial / \partial y\}$ in $F\left(\mathbb{Z}, L^{\sharp}\right)[[X]]$. So there exist $e, f \in A$ such that $e f=q f e, e-1, f$ are nilpotent and such that

$$
\varphi(Q)=e Q \text { and } \varphi(X)=X+f Q,
$$

that are equations in $F\left(\mathbb{Z}, A\left[\left[W_{1}, W_{2}\right]\right]\right)[[X]]$. In particular

$$
\varphi(t)=\varphi(t Q+X)=(e t+f) Q+X=\left(e\left(t+W_{1}\right)+f\right) Q+X,
$$

where we naturally identify rings

$$
F\left(\mathbb{Z}, L^{\sharp}\right)[[X]] \rightarrow F\left(\mathbb{Z}, L^{\natural}\left[\left[W_{1}, W_{2}\right]\right]\right)[[X]] \rightarrow F\left(\mathbb{Z}, A\left[\left[W_{1}, W_{2}\right]\right]\right)[[X]]
$$

through the canonical maps.

Then the argument in the commutative case allows us to show that there exists a power series $b_{0}\left(W_{1}\right) \in A\left[\left[W_{1}\right]\right]$ such that

$$
\varphi\left(Y_{0}\right)=\sum_{n=0}^{\infty} X^{n}\left(\begin{array}{l}
\alpha \\
n
\end{array}\right)_{q}\left(e\left(t+W_{1}\right)+f\right)^{-n} Q^{\alpha-n} b_{0}\left(W_{1}\right) .
$$


such that all the coefficients of the power series $b_{0}\left(W_{1}\right)-1$ are nilpotent. As we deal with the not necessarily commutative algebra $A$, the commutation relation in $L$ gives a constraint. Namely since $\iota(y)=y Y_{0}$ and $t y=y t$ in $L$ so that $\iota(t) \iota(y)=\iota(y) \iota(t)$, we get $\iota(t)\left(y Y_{0}\right)=\left(y Y_{0}\right) \iota(t)$ in $\mathcal{L}$ and $\varphi(t Q+X) \varphi\left(Y_{0}\right)=\varphi\left(Y_{0}\right) \varphi(t Q+X)$. So we consequently have

$$
\mathcal{A Z}_{0}=\mathcal{Z}_{0} \mathcal{A} \quad \text { in } F\left(\mathbb{Z}, A\left[\left[W_{1}, W_{2}\right]\right]\right)[[X]],
$$

setting

$$
\mathcal{A}:=\left(e\left(t+W_{1}\right)+f\right) Q+X, \quad \mathcal{Z}_{0}:=\sum_{n=0}^{\infty} X^{n}\left(\begin{array}{l}
\alpha \\
n
\end{array}\right)_{q}\left(e\left(t+W_{1}\right)+f\right)^{-n} Q^{\alpha-n} b_{0}\left(W_{1}\right) .
$$

LEMMA 5.13. - We have

$$
\left[e\left(t+W_{1}\right)+f, b_{0}\left(W_{1}\right)\right]=0 .
$$

Proof. - This follows from (5.28) and Lemma 5.12.

DEFinition 5.14. - We define a functor

$$
Q G_{2 q}:\left(\mathrm{NCAlg} / L^{\natural}\right) \longrightarrow(\mathrm{Set})
$$

by putting

$$
Q G_{2 q}(A)=\left\{\begin{array}{l|l}
\left(\left[\begin{array}{ll}
e & f \\
0 & 1
\end{array}\right], b\left(W_{1}\right)\right) & \begin{array}{l}
e, f \in A, \text { ef }=\text { qfe }, \\
e \text { is invertible in } A, b\left(W_{1}\right) \in A\left[\left[W_{1}\right]\right], \\
\in M_{2}(A) \times A\left[\left[W_{1}\right]\right]
\end{array} \\
{\left[e\left(t+W_{1}\right)+f, b\left(W_{1}\right)\right]=0}
\end{array}\right\}
$$

for $A \in \mathrm{ob}\left(\mathrm{NCAlg} / l^{\natural}\right)$.

The functor $Q G_{2 q}$ is almost a quantum group in usual sense of the word. See Remark 5.6. We also need the formal completion $\widehat{Q G}_{2 q}$ of the quantum group functor $Q G_{2 q}$ so that

$$
\widehat{Q G}_{2 q}:\left(\mathrm{NCAlg} / L^{\natural}\right) \longrightarrow(\text { Set })
$$

is given by

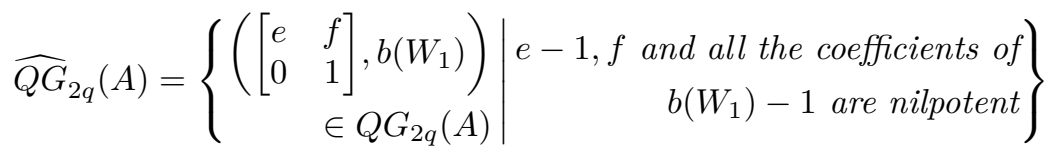

for $A \in \mathrm{ob}\left(\mathrm{NCAlg} / L^{\natural}\right)$.

Studying commutative deformations of the Galois hull $\mathcal{L} / \mathcal{K}$ of $\left(\mathbb{C}\left(t, t^{\alpha}\right), \sigma, \sigma^{-1}, \theta^{*}\right) / \mathbb{C}$, we introduced in Lemma 5.3 the functor $\widehat{G}_{I I}$ and in Lemma 5.5 the functor $\widehat{G}_{2}$. They are isomorphic. The former involves the variable $W_{2}$ but the latter does not. The functor $\widehat{Q G}_{2 q}$ does not involve the 
variable $W_{2}$. As you imagine, we also have another functor $\widehat{Q G}_{I I q}$ equivalent to the functor $\widehat{Q G}_{2 q}$ and involving the variable $W_{2}$.

Using Definition 5.14, we can express what we have shown.

Proposition 5.15. - There exists a functorial inclusion

$$
\mathcal{N C F}_{L / k}(A) \hookrightarrow \widehat{Q G}_{2 q}(A)
$$

sending $\varphi \in \mathcal{N C F}_{L / k}(A)$ to

$$
\left(\left[\begin{array}{ll}
e & f \\
0 & 1
\end{array}\right], b_{0}\left(W_{1}\right)\right) \in \widehat{Q G}_{2 q}(A) .
$$

We show that $\widehat{Q G}_{2 q}$ is a quantum formal group over $L^{\natural}$. In fact, we take two elements

$$
\left(G, \xi\left(W_{1}\right)\right)=\left(\left[\begin{array}{ll}
e & f \\
0 & 1
\end{array}\right], \xi\left(W_{1}\right)\right), \quad\left(H, \eta\left(W_{1}\right)\right)=\left(\left[\begin{array}{ll}
g & h \\
0 & 1
\end{array}\right], \eta\left(W_{1}\right)\right)
$$

of $\widehat{Q G}_{2 q}(A)$ so that $e, f, g, h \in A$ satisfying

$$
\text { ef }=q f e, \quad g h=q h g,
$$

the elements $e-1, g-1$ and $f, h$ are nilpotent and such that

$$
\left[e\left(t+W_{1}\right)+f, \xi\left(W_{1}\right)\right]=0, \quad\left[g\left(t+W_{1}\right)+h, \eta\left(W_{1}\right)\right]=0 .
$$

When the following two sub-sets of the ring $A$

$$
\begin{array}{r}
\{e, f\} \cup\left(\begin{array}{r}
\text { the sub-set consisting of all the coefficients } \\
\text { of the power series } \xi\left(W_{1}\right)
\end{array}\right), \\
\{g, h\} \cup\left(\begin{array}{r}
\text { the sub-set consisting of all the coefficients } \\
\text { of the power series } \eta\left(W_{1}\right)
\end{array}\right),
\end{array}
$$

are element-wisely commutative, we define the product of $\left(G, \xi\left(W_{1}\right)\right)$ and $\left(H, \eta\left(W_{1}\right)\right)$ by

$$
\left(G, \xi\left(W_{1}\right)\right) *\left(H, \eta\left(W_{1}\right)\right):=\left(G H, \xi\left((g-1) t+h+g W_{1}\right) \eta\left(W_{1}\right)\right) .
$$

LEMmA 5.16. - The product $\left(G H, \xi\left((g-1) t+h+g W_{1}\right) \eta\left(W_{1}\right)\right)$ is indeed an element of $\widehat{Q G}_{2 q}(A)$.

Proof. - First of all, we notice that the constant term $(g-1) t+h$ of the linear polynomial in $W_{1}$

$$
(g-1) t+h+g W_{1}
$$

is nilpotent so that we can substitute (5.32) into the power series $\xi\left(W_{1}\right)$. Therefore

$$
\xi\left((g-1) t+h+g W_{1}\right) \eta\left(W_{1}\right)
$$


is a well-determined element of the power series ring $A\left[\left[W_{1}\right]\right]$. We have seen in Section 4 that if $\{e, f\}$ and $\{g, h\}$ are element-wisely commutative, then the product $G H$ of matrices $G, H \in \mathfrak{H}_{q L^{\natural}}(A)$ is in $\mathfrak{H}_{q L^{\natural}}(A)$. Since

$$
G H=\left[\begin{array}{cc}
e g & e h+f \\
0 & 1
\end{array}\right],
$$

it remains to show

$$
\left[e g\left(t+W_{1}\right)+e h+f, \xi\left((g-1) t+h+g W_{1}\right) \eta\left(W_{1}\right)\right]=0 .
$$

The proof of (5.33) is done in several steps.

First we show

$$
\left[\xi\left((g-1) t+h+g W_{1}\right), \eta\left(W_{1}\right)\right]=0 .
$$

This follows, in fact, from the element-wise commutativity of the sub-sets (5.30) and (5.31) above, and the second equation of (5.29).

Second, we show

$$
\left[e g\left(t+W_{1}\right)+e h+f, \xi\left((g-1) t+h+g W_{1}\right)\right]=0 .
$$

To this end, we notice

$$
e g\left(t+W_{1}\right)+e h+f=e\left((g-1) t+h+g W_{1}\right)+e t+f .
$$

So we have to show

$$
\left[e\left((g-1) t+h+g W_{1}\right)+e t+f, \xi\left((g-1) t+h+g W_{1}\right)\right]=0 .
$$

This follows from the first equation of (5.29) and the element-wise commutativity of the sub-sets (5.30) and (5.31).

We prove third

$$
\left[e g\left(t+W_{1}\right)+e h+f, \eta\left(W_{1}\right)\right]=0 .
$$

In fact, by element-wise commutativity of sub-sets (5.30) and (5.31),

$$
\begin{aligned}
{\left[e\left(t+g W_{1}\right)+e h+f, \eta\left(W_{1}\right)\right] } & =\left[e\left(t+g W_{1}\right)+e h, \eta\left(W_{1}\right)\right] \\
& =\left[e\left(t+g W_{1}+h\right), \eta\left(W_{1}\right)\right],
\end{aligned}
$$

which is equal to 0 thanks to element-wise commutativity of the sub-sets (5.30) and (5.31) and the second equality of (5.29).

One can check associativity for the multiplication by a direct calculation. The unit element is given by

$$
\left(I_{2}, 1\right) \in \widehat{Q G}_{2 q}\left(L^{\natural}\right) .
$$

The inverse is given by the formula below. For an element

$$
\begin{gathered}
\left(G, b\left(W_{1}\right)\right)=\left(\left[\begin{array}{ll}
e & f \\
0 & 1
\end{array}\right], b\left(W_{1}\right)\right) \in \widehat{Q G}_{2 q}(A), \\
-1376-
\end{gathered}
$$


we set

$$
\begin{array}{r}
\left(G, b\left(W_{1}\right)\right)^{-1}:=\left(\left[\begin{array}{cc}
e^{-1} & -e^{-1} f \\
0 & 1
\end{array}\right], b\left(e^{-1} W_{1}\right)+\left(e^{-1} t-e^{-1} f\right)^{-1}\right) \\
\in \widehat{Q G}_{2 q^{-1}}(A),
\end{array}
$$

then we have

$$
\left(G, b\left(W_{1}\right)\right)^{-1} *\left(G, b\left(W_{1}\right)\right)=\left(G, b\left(W_{1}\right)\right) *\left(G, b\left(W_{1}\right)\right)^{-1}=\left(I_{2}, 1\right) .
$$

Conjecture 5.17. - If $q$ is not a root of unity, the injection in Proposition 5.15 is bijective for every $A \in \mathrm{ob}\left(\mathrm{NCAlg} / L^{\natural}\right)$.

Proposition 5.18. — Conjecture 5.17 implies Conjecture 5.7.

Proof. - Let us assume Conjecture 5.17. Take an element $\left(e, \xi\left(W_{1}\right)\right) \in$ $\widehat{G}_{2}(A)$ for $A \in \mathrm{ob}\left(\mathrm{Alg} / L^{\natural}\right)$. Since $A$ is commutative, the commutation relation in Lemma 5.13 imposes no condition on $\xi\left(W_{1}\right)$,

$$
\left(e, \xi\left(W_{1}\right)\right)=\left(\left[\begin{array}{ll}
e & 0 \\
0 & 1
\end{array}\right], \xi\left(W_{1}\right)\right) \in \widehat{Q G}_{2 q}(A) .
$$

Conjecture 5.17 says that if $q$ is not a root of unity, $\left(e, \xi\left(W_{1}\right)\right)$ arise from an infinitesimal deformation

$$
\iota: \mathcal{L} \rightarrow F\left(\mathbb{Z}, A\left[\left[W_{1}, W_{2}\right]\right]\right)[[X]] .
$$

Conjecture 5.17 says that we can identify the functor $\mathcal{N C F}_{L / k}$ with the quantum formal group $\widehat{Q G}_{2 q}$. To be more precise, the argument in the First Example studied in 4 allows us to define a formal $\mathbb{C}$-Hopf algebra $\widehat{\mathfrak{I}}_{q}$ and hence

$$
\widehat{\mathfrak{I}}_{q L^{\natural}}:=\widehat{\mathfrak{I}}_{q} \widehat{\otimes}_{\mathbb{C}} L^{\natural},
$$

which is a functor on the category (NCAlg $\left./ L^{\natural}\right)$ so that we have a functorial isomorphism

$$
\widehat{\mathfrak{I}}_{q L^{\natural}}(A) \simeq \widehat{Q G}_{2 q}(A) \quad \text { for every } L^{\natural} \text {-algebra } A \in \operatorname{ob}\left(\mathrm{NCAlg} / L^{\natural}\right) .
$$

Definition 5.19. — We define a functor

$$
\widehat{Q G}_{I I q}:\left(\mathrm{NCAlg} / L^{\natural}\right) \longrightarrow(\text { Set })
$$

by setting

$\widehat{Q G}_{I I q}(A)$

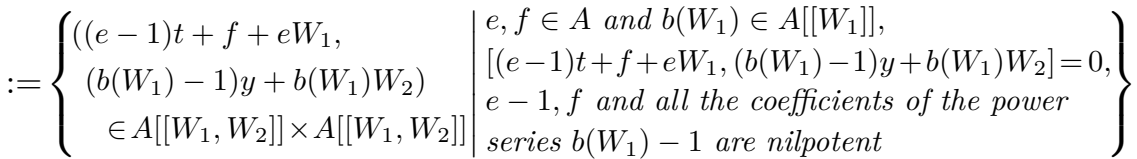

for every $A \in \mathrm{ob}\left(\mathrm{NCAlg} / L^{\natural}\right)$. 
LEMMA 5.20. - The functor $\widehat{Q G}_{I I q}$ is a quantum formal group. Namely, for two elements

$$
\begin{aligned}
& u:=\left(u_{1}, u_{2}\right):=\left((e-1) t+f+e W_{1},\left(b\left(W_{1}\right)-1\right) y+b\left(W_{1}\right) W_{2}\right), \\
& v:=\left(v_{1}, v_{2}\right):=\left((g-1) t+h+g W_{1},\left(c\left(W_{1}\right)-1\right) y+c\left(W_{1}\right) W_{2}\right)
\end{aligned}
$$

of $\widehat{Q G}_{I I q}(A)$, we consider the following two sub-sets of the ring $A$ :

(1) The sub-set $S_{u}$ of the coefficients of the two power series $u_{1}, u_{2}$ of $u$ and

(2) the sub-set $S_{v}$ of the coefficients of the two power series $v_{1}, v_{2}$ in $v$.

If the sets $S_{1}$ and $S_{2}$ are element-wisely commutative, we define their product $u * v$ by

$$
\begin{aligned}
\left((e g-1) t+e h+f+e g W_{1},\left(b\left((g-1) t+h+g W_{1}\right) c\left(W_{1}\right)-1\right) y\right. \\
\left.+b\left((g-1) t+h+g W_{1}\right) c\left(W_{1}\right) W_{2}\right) .
\end{aligned}
$$

that is the composite of coordinate transformations

$$
\begin{aligned}
\left(W_{1}, W_{2}\right) & \longmapsto\left((e-1) t+f+e W_{1},\left(b\left(W_{1}\right)-1\right) y+b\left(W_{1}\right) W_{2}\right) \\
\text { and } \quad\left(W_{1}, W_{2}\right) & \longmapsto\left((g-1) t+h+g W_{1},\left(c\left(W_{1}\right)-1\right) y+c\left(W_{1}\right) W_{2}\right),
\end{aligned}
$$

then the product $u * v$ is an element of $\widehat{Q G}_{I I q}$. The co-unit is given by the identity transformation of $\left(W_{1}, W_{2}\right)$.

The quantum formal group $\widehat{Q G}_{I I q}$ arises as symmetry of the initial conditions of qsi equations.

$$
\begin{aligned}
\sigma(t) & =q t, \quad \sigma\left(t^{\alpha}\right) & =q^{\alpha} t^{\alpha}, \\
\theta^{(1)}(t) & =1, \quad \theta^{(1)}\left(t^{\alpha}\right) & =[\alpha]_{q} t^{\alpha} .
\end{aligned}
$$

Proposition 5.21. - For every algebra $A \in \mathrm{ob}\left(\mathrm{NCAlg} / L^{\natural}\right)$, we have a functorial isomorphism of quantum formal group

$$
\widehat{Q G}_{2 q}(A) \longrightarrow \widehat{Q G}_{I I q}(A)
$$

sending an element

$$
\begin{aligned}
& \left(\left[\begin{array}{ll}
e & f \\
0 & 1
\end{array}\right], b\left(W_{1}\right)\right) \in \widehat{Q G}_{2 q}(A) \\
& \quad \text { to }\left((e-1) t+f+e W_{1}, b\left(W_{1}\right) W_{2}+\left(b\left(W_{1}\right)-1\right) y\right) \in \widehat{Q G}_{I I, q}(A) .
\end{aligned}
$$

Thanks to Propositions 5.15, 5.21 and Conjecture 5.17, we are in the similar situation as in the commutative deformations in 5.2. 
THEOREM 5.22. - We have an inclusion

$$
\mathcal{N C F}_{L / k} \hookrightarrow \widehat{Q G}_{I I q}
$$

of functors on the category (NCAlg $/ L^{\natural}$ ) taking values in the category of sets, where

$$
L / k=\left(\mathbb{C}\left(t, t^{\alpha}\right), \sigma, \sigma^{-1}, \theta^{*}\right) / \mathbb{C} .
$$

Let us assume Conjecture 5.17. Then the inclusion (5.39) is bijection so that we can identify the functors

$$
\mathcal{N C F}_{L / k} \simeq \widehat{Q G}_{I I q}
$$

The quantum formal group $\widehat{Q G}_{I I q}$ operates on the functor $\mathcal{N C F}_{L / k}$ in an appropriate sense, through the initial conditions. (cf. The commutativity condition in Lemma 5.20.) So we may say that the quantum formal Galois group

$$
\operatorname{NC} \operatorname{Inf}-\operatorname{Gal}(L / k) \simeq \widehat{Q G}_{I I q} .
$$

\subsection{Summary on the Galois structures of the field extension $\mathbb{C}\left(t, t^{\alpha}\right) / \mathbb{C}$}

Let us summarize our results on the field extension $\left(\mathbb{C}\left(t, t^{\alpha}\right) / \mathbb{C}\right)$.

(1) Difference field extension $\left(\mathbb{C}\left(t, t^{\alpha}\right), \sigma\right) / \mathbb{C}$. This is a Picard-Vessiot extension with Galois group $\mathbb{G}_{m \mathbb{C}} \times \mathbb{G}_{m \mathbb{C}}$.

(2) Differential field extension $\left(\mathbb{C}\left(t, t^{\alpha}\right), \mathrm{d} / \mathrm{d} t\right) / \mathbb{C}$. This is not a PicardVessiot extension. The Galois group

$$
\operatorname{Inf}-\operatorname{Gal}(L / k):\left(\mathrm{CAlg} / L^{\natural}\right) \longrightarrow(\operatorname{Grp})
$$

is isomorphic to $\widehat{\mathbb{G}}_{m L^{\natural}} \times{ }_{L^{\natural}} \widehat{\mathbb{G}}_{a L^{\natural}}$, where $\widehat{\mathbb{G}}_{m L^{\natural}}$ and $\widehat{\mathbb{G}}_{a L^{\natural}}$ are formal completion of the multiplicative group and the additive group. So as a group functor on the category $\left(\mathrm{CAlg} / L^{\natural}\right)$, we have

$$
\widehat{\mathbb{G}}_{m L^{\natural}}(A)=\{b \in A \mid b-1 \text { is nilpotent }\},
$$

the group law being the multiplication and

$$
\widehat{\mathbb{G}}_{a L^{\natural}}(A)=\{b \in A \mid b \text { is nilpotent }\}
$$

is the additive group for a commutative $L^{\natural}$-algebra $A$.

(3) Commutative deformation of qsi extension $\left(\mathbb{C}\left(t, t^{\alpha}\right), \sigma, \sigma^{-1}, \theta^{*}\right) / \mathbb{C}$. If $q$ is not a root of unity, $\operatorname{Inf}-\operatorname{Gal}(L / k)$ is an infinite-dimensional formal group such that we have

$$
0 \longrightarrow A\left[\widehat{\left.\left[W_{1}\right]\right]} * \operatorname{Inf}-\operatorname{Gal}(L / k)(A) \longrightarrow \widehat{\mathbb{G}}_{m}(A) \longrightarrow 0,\right.
$$


where $A \widehat{\left.A\left[W_{1}\right]\right]} *$ denotes the multiplicative group

$\left\{a \in A\left[\left[W_{1}\right]\right] \mid\right.$ all the coefficients of power series $a-1$ are nilpotent $\}$

modulo Conjecture 5.17.

(4) Non-commutative Galois group. If $q$ is not a root of unity, the Galois group NC Inf-Gal $(L / k)$ is isomorphic to the quantum formal group $\widehat{Q G}_{I I q}$ :

$$
\operatorname{NC} \operatorname{Inf}-\operatorname{Gal}(L / k) \simeq \widehat{Q G}_{I I q}
$$

modulo Conjecture 5.17.

We should be careful about the group law. Quantum formal group structure in $\widehat{Q G}_{I I q}$ coincides with the group structure defined from the initial conditions as in Remark 4.15.

(5) Let us assume $q$ is not a root of unity. If we have a $q$-difference field extension $(L, \sigma) /(k, \sigma)$ such that $t \in L$ with $\sigma(t)=q t$, then we can define the operator $\theta^{(1)}: L \rightarrow L$ by setting

$$
\theta^{(1)}(a):=\frac{\sigma(a)-a}{q t-t}
$$

We also assume the field $k$ is $\theta^{(1)}$ invariant. Defining the operator $\theta^{(n)}: L \rightarrow L$ by

$$
\begin{aligned}
\theta^{(0)} & =\mathrm{Id} \\
\theta^{(n)} & =\frac{1}{[n]_{q} !}\left(\theta^{(1)}\right)^{n}
\end{aligned}
$$

for every positive integer $n$ so that we have a qsi field extension $\left(L, \sigma, \sigma^{-1}, \theta^{*}\right) /\left(k, \sigma, \sigma^{-1}, \theta^{*}\right)$.

Here arises a natural question of comparing the Galois groups of the difference field extension $(L, \sigma) /(k, \sigma)$ and $q s i$ field extension $\left(L, \sigma, \sigma^{-1}, \theta^{*}\right) /\left(k, \sigma, \sigma^{-1}, \theta^{*}\right)$.

As the qsi field extension is constructed from the difference field extension in a more or less trivial way, one might imagine that they coincide or they are not much different.

This contradicts Conjecture 5.17. Let us take our example $\mathbb{C}\left(t, t^{\alpha}\right) / \mathbb{C}$. Assume Conjecture 5.17 is true. Then the Galois group for the qsi extension is $\widehat{Q G}_{I I q L^{\natural}}$ that is infinite-dimensional, whereas the Galois group is of the difference field extension is of dimension 2. 


\section{The Third Example, the $q s i$ field extension}

$$
L / k=\left(\mathbb{C}(t, \log t), \sigma, \sigma^{-1}, \theta^{*}\right) / \mathbb{C}
$$

We assume that $q$ is a complex number not equal to 0 . Let us study the field extension $L / k:=\mathbb{C}(t, \log t) / \mathbb{C}$ from various viewpoints as in Sections 4 and 5 .

\section{1. $q$-difference field extension $\mathbb{C}(t, \log t) / \mathbb{C}$}

We consider $q$-difference operator $\sigma: L \rightarrow L$ such that $\sigma$ is the $\mathbb{C}$-automorphism of the field $L$ satisfying

$$
\sigma(t)=q t \quad \text { and } \quad \sigma(\log t)=\log t+\log q .
$$

It follows from (6.1) that if $q$ is not a root of unity, then the field of constants of the difference field $(\mathbb{C}(t, \log t), \sigma)$ is $\mathbb{C}$ and hence $(\mathbb{C}(t, \log t), \sigma) / \mathbb{C}$ is a Picard-Vessiot extension with Galois group $\mathbb{G}_{m \mathbb{C}} \times_{\mathbb{C}} \mathbb{G}_{a \mathbb{C}}$.

\subsection{Differential field extension $(\mathbb{C}(t, \log t), \mathrm{d} / \mathrm{d} t) / \mathbb{C}$}

As we have

$$
\frac{\mathrm{d} t}{\mathrm{~d} t}=1 \quad \text { and } \quad \frac{\mathrm{d} \log t}{\mathrm{~d} t}=\frac{1}{t},
$$

both differential field extensions $\mathbb{C}(t, \log t) / \mathbb{C}(t)$ and $\mathbb{C}(t) / \mathbb{C}$ are PicardVessiot extensions with Galois group $\mathbb{G}_{a \mathbb{C}}$. The differential extension $\mathbb{C}(t, \log t) / \mathbb{C}$ is not, however, a Picard-Vessiot extension. Therefore, we need general differential Galois theory [22] to speak of the Galois group of the differential field extension $\mathbb{C}(t, \log t) / \mathbb{C}$. The universal Taylor morphism

$$
\iota: L \rightarrow L^{\natural}[[X]]
$$

sends

$$
\begin{aligned}
\iota(t) & =t+X, \\
\iota(\log t) & =\log t+\sum_{n=0}^{\infty}(-1)^{n+1} \frac{1}{n}\left(\frac{X}{t}\right)^{n} \in L^{\natural}[[X]] .
\end{aligned}
$$

Writing $\log t$ by $y$, we take $\partial / \partial t, \partial / \partial y$ as a basis of $L^{\natural}=\mathbb{C}(t, y)^{\natural}$-vector space $\operatorname{Der}\left(L^{\natural} / k^{\natural}\right)$ of $k^{\natural}$-derivations of $L^{\natural}$. It follows from (6.2), (6.3) that

$\mathcal{L}=$ a localization of the algebra $L^{\sharp}\left[t+X, \sum_{n=1}^{\infty}(-1)^{n+1} \frac{1}{n}\left(\frac{X}{t}\right)^{n}\right] \subset L^{\sharp}[[X]]$. 
We argue as in 4.3.1 and Section 5. For a commutative algebra $A \in$ $\mathrm{ob}\left(\mathrm{CAlg} / L^{\natural}\right)$ and $\varphi \in \mathcal{F}_{L / k}(A)$, there exist nilpotent elements $a, b \in A$ such that

$$
\begin{aligned}
\varphi(t+X) & =t+W_{1}+X+a, \\
\varphi\left(\sum_{n=1}^{\infty}(-1)^{n+1} \frac{1}{n}\left(\frac{X}{t+W_{1}}\right)^{n}\right) & =\sum_{n=1}^{\infty}(-1)^{n+1} \frac{1}{n}\left(\frac{X}{t+W_{1}+a}\right)^{n}+b .
\end{aligned}
$$

Therefore we arrived at the dynamical system

$$
\left\{\begin{array} { l } 
{ t , } \\
{ y , }
\end{array} \longmapsto \left\{\begin{array}{l}
\phi(t)=t+X+W_{1}+a, \\
\phi(y)=y+\sum_{n=1}^{\infty}(-1)^{n+1} \frac{1}{n}\left(\frac{X}{t+W_{1}+a}\right)^{n}+b .
\end{array}\right.\right.
$$

In terms of initial conditions, dynamical system (6.4) reads

$$
\left(\begin{array}{l}
t \\
y
\end{array}\right) \mapsto\left(\begin{array}{l}
t+a \\
y+b
\end{array}\right)
$$

where $a, b$ are nilpotent elements of $A$. So we conclude

$$
\operatorname{Inf}-\operatorname{Gal}(L / k)(A)=\widehat{\mathbb{G}}_{a}(A) \times \widehat{\mathbb{G}}_{a}(A)
$$

for every commutative $L^{\natural}$-algebra $A$. Consequently we get

$$
\operatorname{Inf}-\operatorname{Gal}(L / k) \simeq\left(\widehat{\mathbb{G}}_{a \mathbb{C}} \times \widehat{\mathbb{G}}_{a \mathbb{C}}\right) \otimes_{\mathbb{C}} L^{\natural} .
$$

\section{3. qsi field extension $\left(\mathbb{C}(t, \log t), \sigma, \sigma^{-1}, \theta^{*}\right) / \mathbb{C}$}

For the automorphism $\sigma: \mathbb{C}(t, \log t) \rightarrow \mathbb{C}(t, \log t)$ in Subsection 6.1 we set

$$
\theta^{(0)}=\operatorname{Id}_{\mathbb{C}(t, \log t)} \quad \text { and } \quad \theta^{(1)}=\frac{\sigma-\operatorname{Id}_{\mathbb{C}(t, \log t)}}{(q-1) t}
$$

so that $\theta^{(1)}: \mathbb{C}(t, \log t) \rightarrow \mathbb{C}(t, \log t)$ is a $\mathbb{C}$-linear map. We further introduce

$$
\theta^{(i)}:=\frac{1}{[i]_{q} !}\left(\theta^{(1)}\right)^{i}: \mathbb{C}(t, \log t) \longrightarrow \mathbb{C}(t, \log t)
$$

that is a $\mathbb{C}$-linear map for $i=1,2,3, \ldots$ Hence if we denote the set $\left\{\theta^{(i)}\right\}_{i \in \mathbb{N}}$ by $\theta^{*}$, then $\left(\mathbb{C}(t, \log t), \sigma, \sigma^{-1}, \theta^{*}\right)$ is a $q s i$ ring.

The universal Hopf morphism

$$
\iota: \mathbb{C}(t, \log t) \rightarrow F\left(\mathbb{Z}, L^{\sharp}\right)[[X]]
$$

sends, by Proposition 3.11, $t$ and $y$ respectively to

$$
\begin{aligned}
& \iota(t)=t Q+X \\
& \iota(y)=y+(\log q) Z+\frac{\log q}{q-1} \sum_{n=1}^{\infty} X^{n}(-1)^{n+1} \frac{1}{[n]_{q} q^{n(n-1) / 2}}(t Q)^{-n}
\end{aligned}
$$


that we identify with

$$
y+W_{2}+(\log q) Z+\frac{\log q}{q-1} \sum_{n=1}^{\infty} X^{n}(-1)^{n+1} \frac{1}{[n]_{q} q^{n(n-1) / 2}}\left(t+W_{1}\right)^{-n} Q^{-n}
$$

that is an element of $F\left(\mathbb{Z}, L^{\natural}\left[\left[W_{1}, W_{2}\right]\right]\right)[[X]]$, where we set

$$
Z:=\left[\begin{array}{llllll}
\cdots & -1 & 0 & 1 & 2 & \cdots \\
\cdots & -1 & 0 & 1 & 2 & \cdots
\end{array}\right] \in F(\mathbb{Z}, \mathbb{Z}) .
$$

In particular we have

$$
\frac{\partial \iota(y)}{\partial W_{2}}=1
$$

We identify further $t \in L^{\sharp}$ with $t+W_{1} \in L^{\natural}\left[\left[W_{1}, W_{2}\right]\right]$ and hence

$$
\sum_{n=1}^{\infty} X^{n}(-1)^{n+1} \frac{1}{[n]_{q} q^{n(n-1) / 2}}(t Q)^{-n} \in F\left(\mathbb{Z}, L^{\sharp}\right)[[X]]
$$

with

$$
\sum_{n=1}^{\infty} X^{n}(-1)^{n+1} \frac{1}{[n]_{q} q^{n(n-1) / 2}}\left(t+W_{1}\right)^{-n} Q^{-n} \in F\left(\mathbb{Z}, L^{\natural}\left[\left[W_{1}, W_{2}\right]\right]\right)[[X]] .
$$

6.3.1. Commutative deformations $\mathcal{C F}_{L / k}$ for $\left(\mathbb{C}(t, \log t), \sigma, \sigma^{-1}, \theta^{*}\right) / \mathbb{C}$

Now the argument of Section 5 allows us to describe infinitesimal deformations on the category of commutative $L^{\natural}$-algebras (CAlg $/ L^{\natural}$ ). Let $\varphi: \mathcal{L} \rightarrow F\left(\mathbb{Z}, A\left[\left[W_{1}, W_{2}\right]\right]\right)[[X]]$ be an infinitesimal deformation of the canonical morphism $\iota: \mathcal{L} \rightarrow F\left(\mathbb{Z}, A\left[\left[W_{1}, W_{2}\right]\right]\right)[[X]]$ for $A \in \mathrm{ob}\left(\mathrm{CAlg} / L^{\natural}\right)$. Then there exist $e \in A$ such that $e-1$ is nilpotent and such that

$$
\varphi\left(\left(t+W_{1}\right) Q+X\right)=e\left(t+W_{1}\right) Q+X,
$$

as we learned in the First Example. To determine the image $\mathcal{Z}:=\varphi(y)$, we argue as in the Second Example. We have

$$
\begin{aligned}
\sigma(y) & =y+\log q, \\
\theta^{(1)}(y) & =\frac{\log q}{(q-1) t} .
\end{aligned}
$$

Since the deformation $\varphi$ is qsi morphism, the two equations above give us relations

$$
\begin{aligned}
\widehat{\Sigma}(\mathcal{Z}) & =\mathcal{Z}+\log q \\
\widehat{\Theta}^{(1)}(\mathcal{Z}) & =\frac{\log q}{(q-1)\left(\left(t+W_{1}\right) e Q+X\right)} . \\
& -1383-
\end{aligned}
$$


We determine the expansion of the element $\mathcal{Z}$ :

$$
\mathcal{Z}=\sum_{n=0}^{\infty} X^{n} a_{n} \in F\left(\mathbb{Z}, A\left[\left[W_{1}, W_{2}\right]\right]\right)[[X]]
$$

so that

$$
a_{n} \in F\left(\mathbb{Z}, A\left[\left[W_{1}, W_{2}\right]\right]\right) \text { for every } n \in \mathbb{N} .
$$

It follows from (6.5) and (6.8)

$$
a_{0}=y+W_{2}+b\left(W_{1}\right)+(\log q) N \in F\left(\mathbb{Z}, A\left[\left[W_{1}, W_{2}\right]\right]\right),
$$

where $b\left(W_{1}\right)$ is an element of $A\left[\left[W_{1}\right]\right]$ such that all the coefficients of the power series $b\left(W_{1}\right)$ are nilpotent. On the other hand (6.9) tells us

$$
\begin{aligned}
a_{1} & =\frac{\log q}{q-1} \frac{1}{\left(t+W_{1}\right) e Q}, \\
a_{n+1} & =-\frac{[n]_{q}}{[n+1]_{q}} \frac{1}{\left(t+W_{1}\right) e Q q^{n}} a_{n} \quad \text { for } n \geqslant 1 .
\end{aligned}
$$

Hence

$$
a_{n}=(-1)^{n+1}\left(\frac{\log q}{q-1}\right) \frac{1}{[n]_{q} q^{n(n-1) / 2}}\left(t+W_{1}\right)^{-n}(e Q)^{-n} \quad \text { for } n \geqslant 1 .
$$

So we get

$$
\begin{aligned}
\mathcal{Z}=y+ & W_{2}+b\left(W_{1}\right)+(\log q) N \\
& +\frac{\log q}{q-1} \sum_{n=1}^{\infty} X^{n}(-1)^{n+1} \frac{1}{[n]_{q} q^{n(n-1) / 2}}\left(t+W_{1}\right)^{-n}(e Q)^{-n},
\end{aligned}
$$

which is an element of $F\left(\mathbb{Z}, A\left[\left[W_{1}, W_{2}\right]\right]\right)[[X]]$.

Proposition 6.1. - For every commutative $L^{\natural}$-algebra $A \in \mathrm{ob}\left(\mathrm{CAlg} / L^{\natural}\right)$, We have a functorial injection

$$
\mathcal{C F}_{L / k}(A) \longrightarrow \widehat{G}_{3}(A):=\left\{\begin{array}{l|l}
\left(e, b\left(W_{1}\right)\right) & e-1 \text { and all the coefficients } \\
\in A \times A\left[\left[W_{1}\right]\right] & \text { of } b\left(W_{1}\right) \text { are nilpotent }
\end{array}\right\}
$$

sending an element

$$
\varphi \in \mathcal{C F}_{L / k}(A) \text { to }\left(e, b\left(W_{1}\right)\right) \in \widehat{G}_{3}(A) .
$$

CONJECTURe 6.2. - If $q$ is not a root of unity, then the injection in Proposition 6.1 is a bijection.

$\widehat{G}_{3}$ is a group functor on $\left(\mathrm{CAlg} / L^{\natural}\right)$. In fact, for $A \in \mathrm{ob}\left(\mathrm{Alg} / L^{\natural}\right)$, we define the product of two elements

$$
\left(e, b\left(W_{1}\right)\right),\left(g, c\left(W_{1}\right)\right) \in \widehat{G}_{3}(A)
$$


by

$$
\left(e, b\left(W_{1}\right)\right) *\left(g, c\left(W_{1}\right)\right):=\left(e g, b\left((g-1) t+g W_{1}\right)+c\left(W_{1}\right)\right) .
$$

Then, the product is, in fact, an element of $\widehat{G}_{3}(A)$, the product is associative, the unit element of the group law is $\left(I_{2}, 0\right) \in \widehat{G}_{3}(A)$ and the inverse $\left(e, b\left(W_{1}\right)\right)^{-1}=\left(e^{-1},-b\left(e^{-1} W_{1}+\left(e^{-1}-1\right) t\right)\right)$.

So if Conjecture 6.2 is true, we have a splitting exact sequence

$$
0 \longrightarrow A\left[\left[W_{1}\right]\right]_{+} \longrightarrow \operatorname{Inf}-\operatorname{Gal}(L / k)(A) \longrightarrow \widehat{\mathbb{G}}_{m L} \text { घ }(A) \longrightarrow 1,
$$

where $A\left[\left[W_{1}\right]\right]_{+}$denote the additive group of the power series in $A\left[\left[W_{1}\right]\right]$ whose coefficients are nilpotent element.

\subsubsection{Non-commutative deformations $\mathcal{N C F}_{L / k}$ for} $\left(C(t, \log t), \sigma, \sigma^{-1}, \theta^{*}\right) / \mathbb{C}$

The arguments in Section 5 allows us to prove analogous results on the non-commutative deformations for the $q s i$ field extension $(\mathbb{C}(t, \log t), \sigma$, $\left.\sigma^{-1}, \theta^{*}\right) / \mathbb{C}$. We write assertions without giving detailed proofs. For, since the proofs are same, it is easy to find complete proofs.

As in the Second Example, doing calculations $(6.10), \ldots,(6.13)$ in the non-commutative case, we can determine the set $\mathcal{N} \mathcal{C F}\left(\mathbb{C}(t, \log t), \sigma, \sigma^{-1}, \theta^{*}\right) / \mathbb{C}(A)$.

Proposition 6.3. - For a not necessarily commutative $L^{\natural}$-algebra $A \in$ ob $\left(\mathrm{NCAlg} / \mathrm{L}^{\natural}\right)$, we can describe an infinitesimal deformation

$$
\varphi \in \mathcal{N C F}_{\left(\mathbb{C}(t, \log t), \sigma, \sigma^{-1}, \theta^{*}\right) / \mathbb{C}}(A) .
$$

Namely putting $y:=\log t$, we have

$$
\begin{aligned}
\varphi(t)= & \left(e\left(t+W_{1}\right)+f\right) Q+X \\
\varphi(y)=y & +W_{2}+b\left(W_{1}\right)+(\log q) Z \\
& \quad+\frac{\log q}{q-1} \sum_{n=1}^{\infty} X^{n}(-1)^{n+1} \frac{1}{[n]_{q} q^{n(n-1) / 2}}\left[e\left(t+W_{1}\right)+f\right]^{-n} Q^{-n}
\end{aligned}
$$

that are elements of $F\left(\mathbb{Z}, A\left[\left[W_{1}, W_{2}\right]\right]\right)[[X]]$, where $e, f \in A$ and $b\left(W_{1}\right) \in$ $A\left[\left[W_{1}\right]\right]$ satisfying the following conditions.

(1) ef $=q f e$.

(2) $e-1$ and $f$ are nilpotent elements of $A$.

(3) All the coefficients of the power series $b\left(W_{1}\right)$ are nilpotent.

(4) $\left[e\left(t+W_{1}\right)+f, b\left(W_{1}\right)\right]=0$.

The commutativity condition (4) comes from the commutativity relation between the elements $t$ and $y=\log t$ in the field $L$. 
Definition 6.4. - We introduce a functor

$$
\widehat{Q G}_{3 q}:\left(\mathrm{NCAlg} / L^{\natural}\right) \rightarrow(\text { Set })
$$

by setting

$$
\begin{aligned}
& \widehat{Q G}_{3 q}(A) \\
& :=\left\{\begin{array}{l|l}
\left(G, \xi\left(W_{1}\right)\right) \in \mathfrak{H}_{q L^{\natural}}(A) \times A\left[\left[W_{1}\right]\right] & \begin{array}{c}
(1) G=\left[\begin{array}{l}
\text { e } \\
0
\end{array}\right] \in \widehat{\mathfrak{H}}_{q}(A) \text { so that } \\
\text { ef }=q f e, e-1, f \in A \text { are nilpotent. } \\
(2) \text { All the coefficients of } \xi\left(W_{1}\right) \\
\text { are nilpotent. } \\
(3)\left[e\left(t+W_{1}\right)+f, \xi\left(W_{1}\right)\right]=0 .
\end{array}
\end{array}\right\}
\end{aligned}
$$

$\widehat{Q G}_{3 q}$ is a quantum formal group. Namely, for

$$
\left(G, \xi\left(W_{1}\right)\right),\left(H, \eta\left(W_{1}\right)\right) \in \widehat{Q G}_{3 q}(A)
$$

such that the two sub-sets

\{all the entries of matrix $G$, all the coefficients of the power series $\left.\xi\left(W_{1}\right)\right\}$, \{all the entries of matrix $H$, all the coefficients of the power series $\left.\eta\left(W_{1}\right)\right\}$ of $A$ are element-wisely commutative, we define their product by

$$
\left(G, \xi\left(W_{1}\right)\right) *\left(H, \eta\left(W_{1}\right)\right):=\left(G H, \xi\left((g-1) t+h+g W_{1}\right)+\eta\left(W_{1}\right)\right),
$$

where

$$
H=\left[\begin{array}{ll}
g & h \\
0 & 1
\end{array}\right] .
$$

Then, the argument of Lemma 5.16 shows that the product of two elements is, in fact, an element in the set $\widehat{Q G}_{3 q}(A)$ and the product is associative. The unit element is $\left(I_{2}, 0\right) \in \widehat{Q G}_{3 q}(A)$. The inverse

$$
\left(G, \xi\left(W_{1}\right)\right)^{-1}=\left(G^{-1},-\xi\left(\left(e^{-1}-1\right) t-e^{-1} f+e^{-1} W_{1}\right) \in \widehat{Q G}_{3 q^{-1}}(A),\right.
$$

where

$$
G=\left[\begin{array}{ll}
e & f \\
0 & 1
\end{array}\right] .
$$

Proposition 6.5. - We have a functorial injection

$$
\begin{gathered}
\mathcal{N C F}_{L / k}(A) \longrightarrow \widehat{Q G}_{3 q}(A) \\
-1386-
\end{gathered}
$$


that sends $\varphi \in \mathcal{N C F}_{L / k}(A)$ to $\left(\left[\begin{array}{ll}e & f \\ 0 & 1\end{array}\right], b\left(W_{1}\right)\right)$. Here

$$
\begin{aligned}
& \varphi\left(\left(t+W_{1}\right) Q+X\right)=\left(e\left(t+W_{1}\right)+f\right) Q+X \\
& \varphi(\iota(y))= \varphi\left(y+W_{2}+\log q N\right. \\
&\left.+\frac{\log q}{q-1} \sum_{n=1}^{\infty} X^{n}(-1)^{n+1} \frac{1}{[n]_{q} q^{n(n-1) / 2}}\left(\left(t+W_{1}\right)^{-n} Q^{-n}\right)\right) \\
&= y+W_{2}+b\left(W_{1}\right)+\log q N \\
&+\frac{\log q}{q-1} \sum_{n=1}^{\infty} X^{n}(-1)^{n+1} \frac{1}{[n]_{q} q^{n(n-1) / 2}}\left(e\left(t+W_{1}\right)+f\right)^{-n} Q^{-n} .
\end{aligned}
$$

We also have a Conjecture.

Conjecture 6.6. - If $q$ is not a root of unity, then the injection in Proposition 6.5 is a bijection. So

$$
\mathcal{N C F}_{L / k} \simeq \widehat{Q G}_{3 q}
$$

Remark 6.7. - The argument in 5.3 allows us to prove that Conjecture 6.6 implies Conjecture 6.2.

We can also define the quantum formal group $\widehat{Q G}_{I I I q}$ in terms of noncommutative coordinate transformations as in the Second Example.

Definition 6.8. - We define a functor

$$
\widehat{Q G}_{I I I q}:\left(\mathrm{NCAlg} / L^{\natural}\right) \longrightarrow(\text { Set })
$$

by setting

$\widehat{Q G}_{I I I q}(A)$

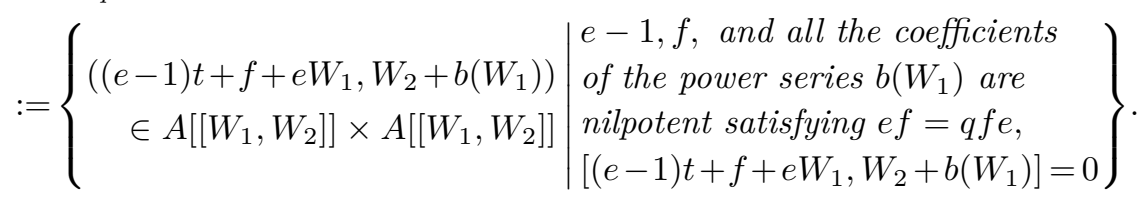

We regard an element

$$
\varphi=\left(\varphi_{1}\left(W_{1}, W_{2}\right), \varphi_{2}\left(W_{1}, W_{2}\right)\right) \in \widehat{Q G}_{I I I q}(A)
$$

as an infinitesimal coordinate transformation $\Phi$

$$
\left(W_{1}, W_{2}\right) \mapsto\left(\varphi_{1}\left(W_{1}, W_{2}\right), \varphi_{2}\left(W_{1}, W_{2}\right)\right)
$$

with non-commutative coefficients. The product in the quantum formal group $\widehat{Q G}_{I I q}$ is the composition of coordinate transformations if they satisfy a commutation relation so that the product is defined. To be more concrete, let

$$
\left((e-1) t+f+e W_{1}, W_{2}+b\left(W_{1}\right)\right) \text { and }\left((g-1) t+h+g W_{1}, W_{2}+c\left(W_{1}\right)\right)
$$


be two elements of $\widehat{Q G}_{I I I q}(A)$ such that the following two sub-sets of the ring $A$ is element-wisely commutative:

(1) $\{e, f\} \cup\left(\right.$ the set of coefficients of the power series $\left.b\left(W_{1}\right)\right)$,

(2) $\{g, h\} \cup$ (the set of coefficients of the power series $\left.c\left(W_{1}\right)\right)$,

then the product is

$$
\begin{aligned}
& \left((e-1) t+f+e W_{1}, W_{2}+b\left(W_{1}\right)\right) *\left((g-1) t+h+g W_{1}, W_{2}+c\left(W_{1}\right)\right) \\
& \quad=\left((e g-1) t+e h+f+e g W_{1}, W_{2}+b\left((g-1) t+h+g W_{1}\right)+c\left(W_{1}\right)\right)
\end{aligned}
$$

which is certainly an element of $\widehat{Q G}_{I I I q}(A)$.

Though we reversed the procedure, the quantum formal group $\widehat{Q G}_{3 q}$ arises from $\widehat{Q G}_{I I I q}$ and we arrived at the last object as a natural extension of Lie-Ritt functor in [22] of coordinate transformations in the space of initial conditions.

Proposition 6.9. - For every algebra $A \in \mathrm{ob}\left(\mathrm{NCAlg} / L^{\natural}\right)$, we have a functorial isomorphism of quantum formal groups

$$
\widehat{Q G}_{3 q}(A) \longrightarrow \widehat{Q G}_{I I I q}(A)
$$

sending an element

$\left(\left[\begin{array}{ll}e & f \\ 0 & 1\end{array}\right], b\left(W_{1}\right)\right) \in \widehat{Q G}_{3 q}(A)$ to $\left((e-1) t+f+e W_{1}, W_{2}+b\left(W_{1}\right)\right) \in \widehat{Q G}_{I I I q}(A)$.

Looking at Propositions 6.5, 6.9 and Conjecture 6.6, we find that we are in the same situation as in 5.3, where we studied non-commutative deformations of the Second Example.

THEOREM 6.10. - We have an inclusion

$$
\mathcal{N C F}_{L / k} \hookrightarrow \widehat{Q G}_{I I I q}
$$

of functors on the category (NCAlg $/ L^{\natural}$ ) taking values in the category of sets, where

$$
L / k=\left(\mathbb{C}(t, \log t), \sigma, \sigma^{-1}, \theta^{*}\right) / \mathbb{C} .
$$

If we assume Conjecture 6.6, then the inclusion (6.17) is bijection so that we can identify the functors

$$
\mathcal{N C \mathcal { F } _ { L / k }} \simeq \widehat{Q G}_{I I I q}
$$

The quantum formal group $\widehat{Q G}_{I I I q}$ operates on the functor $\mathcal{N C F}_{L / k}$ in an appropriate sense, through the initial conditions. (cf. The commutativity condition in Definition 6.8.) So we may say that the quantum formal Galois group

$$
\operatorname{NC} \operatorname{Inf}-\operatorname{Gal}(L / k) \simeq \widehat{Q G}_{I I I q} .
$$




\subsection{Summary on the Galois structures of the field extension $\mathbb{C}(t, \log t) / \mathbb{C}$}

Let us summarize our results on the field extension $\mathbb{C}(t, \log t) / \mathbb{C}$.

(1) Difference field extension $(\mathbb{C}(t, \log t), \sigma) / \mathbb{C}$. This is a Picard-Vessiot extension with Galois group $\mathbb{G}_{a \mathbb{C}} \times \mathbb{G}_{a \mathbb{C}}$.

(2) Differential field extension $(\mathbb{C}(t, \log t), \mathrm{d} / \mathrm{d} t) / \mathbb{C}$. This is not a PicardVessiot extension. The Galois group

$$
\operatorname{Inf-Gal}(L / k):\left(\mathrm{CAlg} / L^{\natural}\right) \longrightarrow(\operatorname{Grp})
$$

is isomorphic to $\widehat{\mathbb{G}}_{a L^{\natural}} \times_{L^{\natural}} \widehat{\mathbb{G}}_{a L^{\natural}}$, where $\widehat{\mathbb{G}}_{a L^{\natural}}$ is the formal completion of the additive group. So as a group functor on the category $\left(\mathrm{CAlg} / L^{\natural}\right)$, we have

$$
\widehat{\mathbb{G}}_{a L^{\natural}}(A)=\{b \in A \mid b \text { is nilpotent }\},
$$

the group law being the addition and hence

$\operatorname{Inf}-\operatorname{Gal}(L / k)(A)=\{(a, b) \mid a, b$ are nilpotent elements of $A\}$

for a commutative $L^{\natural}$-algebra $A$.

(3) Commutative deformations of $q s i$ extension $\left(\mathbb{C}(t, \log t), \sigma, \sigma^{-1}, \theta^{*}\right) / \mathbb{C}$. If $q$ is not a root of unity, $\operatorname{Inf}-\operatorname{Gal}(L / k)$ is an infinite-dimensional formal group such that we have a splitting sequence

$$
0 \longrightarrow A\left[\left[W_{1}\right]\right]_{+} \longrightarrow \operatorname{Inf}-\operatorname{Gal}(L / k)(A) \longrightarrow \widehat{\mathbb{G}}_{m}(A) \longrightarrow 0,
$$

where $A\left[\left[W_{1}\right]\right]_{+}$denotes the additive group

$\left\{a \in A\left[\left[W_{1}\right]\right] \mid\right.$ all the coefficients of power series $a$ are nilpotent $\}$

modulo Conjecture 6.6.

(4) Non-commutative Galois group. If $q$ is not a root of unity, the Quantum Galois group NC $\operatorname{Inf}-\operatorname{Gal}(L / k)$ is isomorphic to a quantum formal group $\widehat{Q G}_{I I I q}$ :

$$
\operatorname{NC} \operatorname{Inf}-\operatorname{Gal}(L / k) \simeq \widehat{Q G}_{I I I q} .
$$

modulo Conjecture 6.6.

We should be careful about the group law. Quantum formal group structure in $\widehat{Q G}_{I I I q}$ coincides with the group structure defined from the initial conditions as in Proposition 6.9. 


\section{General scope of quantized Galois theory for qsi field extensions}

After we worked with three examples of qsi field extensions

$$
\mathbb{C}(t) / \mathbb{C}, \quad \mathbb{C}\left(t, t^{\alpha}\right) / \mathbb{C} \quad \text { and } \mathbb{C}(t, \log t) / \mathbb{C},
$$

there arises naturally, in our mind, the idea of formulating general quantized Galois theory for qsi field extensions. The simplest differential Example 2.3 is also very inspiring. We are going to give evidence that a general theory should exist.

\subsection{Outline of the theory}

Let $L / k$ be a qsi field extension such that the abstract field extension $L^{\natural} / k^{\natural}$ is of finite type. Galois theory for qsi filed extensions is a particular case of Hopf Galois theory in Section 3. So as we learned in 3.2.6, we have the universal Hopf morphism

$$
\iota: L \longrightarrow F\left(\mathbb{Z}, L^{\natural}\right)[[X]] .
$$

We choose a basis

$$
\left\{D_{1}, D_{2}, \ldots, D_{d}\right\}
$$

of mutually commutative derivations of the $L^{\natural}$-vector space $\operatorname{Der}\left(L^{\natural} / k^{\natural}\right)$ of $k^{\natural}$-derivations of $L^{\natural}$. We constructed the Galois hull $\mathcal{L} / \mathcal{K}$ in Definition 3.13. So we have the canonical morphism

$$
\iota: \mathcal{L} \longrightarrow F\left(\mathbb{Z}, L^{\natural}\left[\left[W_{1}, W_{2}, \ldots, W_{d}\right]\right]\right)[[X]] .
$$

The rings $\mathcal{L}$ and $\mathcal{K}$ are invariant under the set of operators

$$
\mathcal{D}:=\left\{\widehat{\Sigma}, \widehat{\Theta}^{*}, \frac{\partial}{\partial W_{i}}(1 \leqslant i \leqslant d)\right\}
$$

on $F\left(\mathbb{Z}, L^{\natural}[[W]]\right)[[X]]$.

In general, the Galois hull $\mathcal{L} / \mathcal{K}$ is not commutative. So we measure it by infinitesimal deformations of the canonical morphism (7.1) over the category (NCAlg $/ L^{\natural}$ ) of not necessarily commutative $L^{\natural}$-algebras. We set in Definition 3.15

$$
\begin{aligned}
& \mathcal{N C F}_{L / k}(A) \\
& =\left\{\begin{array}{l|l}
\varphi: \mathcal{L} \rightarrow F(\mathbb{Z}, A[[W]])[[X]] & \begin{array}{l}
\varphi \text { is an infinitesimal deformation over } \mathcal{K} \\
\text { compatible with } \mathcal{D} \text { of canonical } \\
\text { morphism }(7.1)
\end{array} \\
-1390-
\end{array}\right\}
\end{aligned}
$$


so that we get the functor

$$
\mathcal{N C F}_{L / k}:\left(\mathrm{NCAlg} / L^{\natural}\right) \longrightarrow \text { (Set). }
$$

Now we compare the differential case and qsi case to understand their similarity and difference.

(1) Differential case

(a) The Galois hull $\mathcal{L} / \mathcal{K}$ is an extension of commutative algebras.

(b) It is sufficient to consider commutative deformation functor $\mathcal{F}_{L / k}$ of the Galois hull $\mathcal{L} / \mathcal{K}$ over the category $\left(\mathrm{CAlg} / L^{\natural}\right.$ ) of commutative $L^{\natural}$-algebras.

(c) The Galois group $\operatorname{Inf-Gal}(L / k)$ is a kind of generalization of algebraic group. In fact, it is at least a group functor on the category $\left(\mathrm{CAlg} / L^{\natural}\right)$.

(d) Indeed the group functor Inf-Gal $(L / k)$ is given as the functor of automorphisms of the Galois hull $\mathcal{L} / \mathcal{K}$.

(2) qsi case

(a) Galois hull $\mathcal{L} / \mathcal{K}$ is not always an extension of commutative algebras.

(b) We have to consider the non-commutative deformation functor $\mathcal{N C F}_{L / k}$ over the category ( $\mathrm{NCAlg} / L^{\natural}$ ) of not necessarily commutative $L^{\natural}$-algebras.

(c) The Galois group should be a quantum group that we can not interpret in terms of group functor.

The comparison above shows that we have to find a counterpart of (d) in the qsi case. The three examples suggest the following solution.

\section{Solution that we propose}

Let $y_{1}, y_{2}, \ldots, y_{d}$ be a transcendence basis of the abstract field extension $L^{\natural} / k^{\natural}$. We set by

$$
\iota\left(y_{i}\right)=Y_{i}\left(W_{1}, W_{2}, \ldots, W_{d} ; X\right) \in F\left(\mathbb{Z}, L^{\natural}[[W]]\right)[[X]] \quad \text { for } 1 \leqslant i \leqslant d .
$$

\section{Questions 7.1. -}

(1) For an $L^{\natural}$-algebra $A \in \mathrm{ob}\left(\mathrm{NCAlg} / L^{\natural}\right)$, let

$$
f: \mathcal{L} \longrightarrow F(\mathbb{Z}, A[[W]])[[X]]
$$

be an infinitesimal deformation of the canonical morphism $\iota$. Then there exist an infinitesimal coordinate transformation

$$
\Phi=\left(\varphi_{1}(W), \varphi_{2}(W), \ldots, \varphi_{d}(W)\right) \in A\left[\left[W_{1}, W_{2}, \ldots, W_{d}\right]\right]^{d}
$$


with coefficients in the not necessarily commutative algebra $A$ such that

$$
f\left(Y_{i}\right)=Y_{i}(\Phi(W) ; X) \text { for every } 1 \leqslant i \leqslant d .
$$

(2) Assume that Question (1) is affirmatively answered. Then we have a functorial morphism

$$
\mathcal{N C F}_{L / k}(A) \longrightarrow\left\{\Phi \in A[[W]]^{d} \mid \begin{array}{l}
W \mapsto \Phi(W) \text { is an infinitesimal } \\
\text { coordinate transformation }
\end{array}\right\}
$$

sending $f$ to $\Phi$ using the notation of (1). We set

$$
\mathrm{Q} \operatorname{Inf}-\operatorname{Gal}(L / k)(A):=\text { the image of map (7.3) }
$$

so that

$$
\mathrm{Q} \operatorname{Inf}-\operatorname{Gal}(L / k):\left(\mathrm{NCAlg} / L^{\natural}\right) \longrightarrow(\text { Set })
$$

is a functor. Our second question is if the functor $\mathrm{Q} \operatorname{Inf}-\mathrm{Gal}(L / k)$ is a quantum formal group.

(3) Assume that Question (1) has an affirmative answer. Since the identity transformation is in $\mathrm{Q} \operatorname{Inf}-\mathrm{Gal}(L / k)$, Question (2) reduces to the following concrete question. Let $f, g$ be elements of $\mathcal{N C F}_{L / k}(A)$ and let $\Phi$ and $\Psi$ be the corresponding coordinate transformations to $f$ and $g$ respectively. If the set of the coefficients of $\Phi$ and the set of the coefficients of $\Psi$ is element-wisely commutative, then does the composite coordinate transformation $\Phi \circ \Psi$ arise from an infinitesimal deformation $h \in \mathcal{N C F}_{L / k}(A)$ ?

In view of Corollary 9.9, the universal deformation or the universal coaction seems to solve the Questions. It seems that we are very close to the solutions.

\section{Part II. Quantization of Picard-Vessiot theory}

Keeping the notation of the Part I, we denote by $C$ a field of characteristic 0 .

We believed for a long time that it was impossible to quantize PicardVessiot theory, Galois theory for linear difference or differential equations. Namely, there was no Galois theory for linear difference-differential equations, of which the Galois group is a quantum group that is, in general, neither commutative nor co-commutative. Our mistake came from a misunderstanding of preceding works, Hardouin [5] and Masuoka and Yanagawa [14]. They studied linear qsi equations, qsi equations for short, under two assumptions on qsi base field $K$ and qsi module $M$ :

(1) The base field $K$ contains $C(t)$. 
(2) On the $K\left[\sigma, \sigma^{-1}, \theta^{*}\right]$-module $M$ the equality

$$
\theta^{(1)}=\frac{1}{(q-1) t}\left(\sigma-\operatorname{Id}_{M}\right) .
$$

holds. Under these conditions, their Picard-Vessiot extension is realized in the category of commutative qsi algebras. The second assumption seems too restrictive as clearly explained in [14]. If we drop one of these conditions, there are many linear qsi equations whose Picard-Vessiot ring is not commutative and the Galois group is a quantum group that is neither commutative nor co-commutative.

We first analyze in detail, one favorite example (8.6) over the base field $C$, which is equivalent to the non-linear equation in Section 4 . We then quickly study three more example in Section 12. Looking at these examples, the reader's imagination would go far away, as Cartier [3] did it for every qsi linear equation with constant coefficients.

In the favorite example, we have a Picard-Vessiot ring $R$ that is noncommutative, simple qsi ring (Observation 9.3 and Lemma 9.4). The PicardVessiot ring $R$ is a torsor of a quantum group (Observation 9.6). As for equivalence of rigid tensor categories, we note Expectation 11.2 and prove a modified version in Part III. We have a partial Galois correspondence (Observation 11.3). We prove the uniqueness of the Picard-Vessiot ring for certain examples in Section 10. Picard-Vessiot ring is, however, not unique in general as we see in Section 17.

\section{Field extension $C(t) / C$ from classical and quantum viewpoints}

In Section 4, we studied a non-linear qsi equation, which we call qsi equation for short,

$$
\theta^{(1)}(y)=1, \quad \sigma(y)=q y, \quad \sigma^{-1}(y)=q^{-1} y,
$$

where $q$ is an element of the field $C$ not equal to 0 nor 1 . Let $t$ be a variable over the constant base field $C$. We assume to simplify the situation that $q$ is not a root of unity. We denote by $\sigma: C(t) \rightarrow C(t)$ the $C$-automorphism of the field $C(t)$ of rational functions sending $t$ to $q t$. We introduce the $C$-linear operator $\theta^{(1)}: C(t) \rightarrow C(t)$ by

$$
\theta^{(1)}(f(t)):=\frac{f(q t)-f(t)}{(q-1) t} \text { for every } f(t) \in C(t) .
$$

We set

$$
\theta^{(m)}:= \begin{cases}\operatorname{Id}_{C(t)} & \text { for } m=0 \\ \frac{1}{[m]_{q} !}\left(\theta^{(1)}\right)^{m} & \text { for } m=1,2, \ldots\end{cases}
$$


As we assume that $q$ is not a root of unity, the number $[m]_{q}$ in the formula is not equal to 0 and hence the formula determines the family $\theta^{*}=\left\{\theta^{(i)} \mid i \in \mathbb{N}\right\}$ of operators. So $\left(C(t), \sigma, \sigma^{-1}, \theta^{*}\right)$ is a qsi field. See Section 4 and $y=t$ is a solution for system (8.1).

The system (8.1) is non-linear in the sense that for two solutions $y_{1}, y_{2}$ of (8.1), a $C$-linear combination $c_{1} y_{1}+c_{2} y_{2}\left(c_{1}, c_{2} \in C\right)$ is not a solution of the system in general.

However, the system is very close to a linear system. To illustrate this, let us look at the differential field extension $\left(C(t), \partial_{t}\right) /\left(C, \partial_{t}\right)$, where we denote the derivation $\mathrm{d} / \mathrm{d} t$ by $\partial_{t}$ The variable $t \in C(t)$ satisfies a non-linear differential equation

$$
\partial_{t} t-1=0
$$

The differential field extension $\left(C(t), \partial_{t}\right) /\left(C, \partial_{t}\right)$ is, however, the PicardVessiot extension for the linear differential equation

$$
\partial_{t}^{2} t=0 \text {. }
$$

To understand the relation between (8.2) and (8.3), we introduce the 2dimensional $C$-vector space

$$
E:=C t \oplus C \subset C[t] .
$$

The vector space $E$ is closed under the action of the derivation $\partial_{t}$ so that $E$ is a $C\left[\partial_{t}\right]$-module. Solving the differential equation associated with the $C\left[\partial_{t}\right]$-module $E$ is to find a differential algebra $\left(L, \partial_{t}\right) / C$ and a $C\left[\partial_{t}\right]$-module morphism

$$
\varphi: E \rightarrow L \text {. }
$$

Writing $\varphi(t)=f_{1}, \varphi(1)=f_{2}$ that are elements of $L$, we have

$$
\left[\begin{array}{l}
\partial_{t} f_{1} \\
\partial_{t} f_{2}
\end{array}\right]=\left[\begin{array}{ll}
0 & 1 \\
0 & 0
\end{array}\right]\left[\begin{array}{l}
f_{1} \\
f_{2}
\end{array}\right]
$$

Since $\partial_{t} t=1, \partial_{t} 1=0$, in the differential field $\left(C(t), \partial_{t}\right) / C$, we find two solutions ${ }^{t}(t, 1)$ and ${ }^{t}(1,0)$ that are two column vectors in $C(t)^{2}$ satisfying

$$
\partial_{t}\left[\begin{array}{ll}
t & 1 \\
1 & 0
\end{array}\right]=\left[\begin{array}{ll}
0 & 1 \\
0 & 0
\end{array}\right]\left[\begin{array}{ll}
t & 1 \\
1 & 0
\end{array}\right]
$$

and

$$
\left|\begin{array}{ll}
t & 1 \\
1 & 0
\end{array}\right| \neq 0
$$

Namely, $C(t) / C$ is the Picard-Vessiot extension for linear differential equation (8.4).

We can argue similarly for the qsi field extension $\left(C(t), \sigma, \sigma^{-1}, \theta^{*}\right) / C$. You will find a subtle difference between the differential case and the qsi case. Quantization of Galois group arises from here. 
Let us set

$$
M=C t \oplus C \subset C[t]
$$

that is a $C\left[\sigma, \sigma^{-1}, \theta^{*}\right]$-module. To avoid the confusion that you might have in Remark 8.3 below, writing $m_{1}=t$ and $m_{2}=1$, we had better formally write

$$
M=C m_{1} \oplus C m_{2}
$$

as a $C$-vector space on which $\sigma$ and $\theta^{(1)}$ operate by

$$
\begin{gathered}
{\left[\begin{array}{l}
\sigma\left(m_{1}\right) \\
\sigma\left(m_{2}\right)
\end{array}\right]=\left[\begin{array}{ll}
q & 0 \\
0 & 1
\end{array}\right]\left[\begin{array}{l}
m_{1} \\
m_{2}
\end{array}\right], \quad\left[\begin{array}{l}
\sigma^{-1}\left(m_{1}\right) \\
\sigma^{-1}\left(m_{2}\right)
\end{array}\right]=\left[\begin{array}{cc}
q^{-1} & 0 \\
0 & 1
\end{array}\right]\left[\begin{array}{l}
m_{1} \\
m_{2}
\end{array}\right],} \\
{\left[\begin{array}{l}
\theta^{(1)}\left(m_{1}\right) \\
\theta^{(1)}\left(m_{2}\right)
\end{array}\right]=\left[\begin{array}{ll}
0 & 1 \\
0 & 0
\end{array}\right]\left[\begin{array}{l}
m_{1} \\
m_{2}
\end{array}\right] .}
\end{gathered}
$$

Since in (8.6) the first equation is equivalent to the second, we only consider the first and third equations. Solving $C\left[\sigma, \sigma^{-1}, \theta^{*}\right]$-module $M$ is equivalent to find elements $f_{1}, f_{2}$ in a $q s i$ algebra $\left(A, \sigma, \sigma^{-1}, \theta^{*}\right)$ satisfying the system of linear difference-differential equation

$$
\left[\begin{array}{l}
\sigma\left(f_{1}\right) \\
\sigma\left(f_{2}\right)
\end{array}\right]=\left[\begin{array}{ll}
q & 0 \\
0 & 1
\end{array}\right]\left[\begin{array}{l}
f_{1} \\
f_{2}
\end{array}\right], \quad\left[\begin{array}{l}
\theta^{(1)}\left(f_{1}\right) \\
\theta^{(1)}\left(f_{2}\right)
\end{array}\right]=\left[\begin{array}{ll}
0 & 1 \\
0 & 0
\end{array}\right]\left[\begin{array}{l}
f_{1} \\
f_{2}
\end{array}\right]
$$

in the $q s i$ algebra $A$.

LEMMA 8.1. - Let $\left(L, \sigma, \sigma^{-1}, \theta^{*}\right) / C$ be a qsi field extension. If a $2 \times 2$ matrix $Y=\left(y_{i j}\right) \in M_{2}(L)$ satisfies a system of difference-differential equations

$$
\sigma Y=\left[\begin{array}{ll}
q & 0 \\
0 & 1
\end{array}\right] Y \quad \text { and } \quad \theta^{(1)} Y=\left[\begin{array}{ll}
0 & 1 \\
0 & 0
\end{array}\right] Y,
$$

then $\operatorname{det} Y=0$.

Proof. - It follows from (8.8)

$$
\sigma\left(y_{11}\right)=q y_{11}, \quad \sigma\left(y_{12}\right)=q y_{12}, \quad \sigma\left(y_{21}\right)=y_{21}, \quad \sigma\left(y_{22}\right)=y_{22}
$$

and

$$
\theta^{(1)}\left(y_{11}\right)=y_{21}, \quad \theta^{(1)}\left(y_{12}\right)=y_{22}, \quad \theta^{(1)}\left(y_{21}\right)=0, \quad \theta^{(1)}\left(y_{22}\right)=0 .
$$

It follows from (8.9) and (8.10)

$$
\theta^{(1)}\left(y_{11} y_{12}\right)=\theta^{(1)}\left(y_{11}\right) y_{12}+\sigma\left(y_{11}\right) \theta^{(1)}\left(y_{12}\right)=y_{21} y_{12}+q y_{11} y_{22}
$$

and similarly

$$
\theta^{(1)}\left(y_{12} y_{11}\right)=y_{22} y_{11}+q y_{12} y_{21}
$$

As $y_{11} y_{12}=y_{12} y_{11}$, equating (8.11) and (8.12), we get

$$
(q-1)\left(y_{11} y_{22}-y_{12} y_{21}\right)=0
$$

so that $\operatorname{det} Y=0$. 
Corollary 8.2. - Let $\left(K, \sigma, \sigma^{-1}, \theta^{*}\right)$ be a qsi field over $C$. Then the qsi linear equation

$$
\sigma Y=\left[\begin{array}{ll}
q & 0 \\
0 & 1
\end{array}\right] Y \quad \text { and } \quad \theta^{(1)} Y=\left[\begin{array}{ll}
0 & 1 \\
0 & 0
\end{array}\right] Y
$$

admits no qsi extension $L$ over $K$ with the following properties, which would deserve to call it a qsi Picard-Vessiot extension or simply a Picard-Vessiot extension:

(1) $L$ is generated over $K$ by the entries of some solution matrix $Y \in$ $\mathrm{GL}_{2}(L)$ to (8.13), and

(2) the fields of constants in $L$ and in $K$ coincide.

Proof. - This is a consequence of Lemma 8.1.

Remark 8.3. - We note that Corollary 8.2 is compatible with Hardouin [5, Remark 4.4 and Theorem 4.7]. See also Masuoka and Yanagawa [14]. They assure the existence of Picard-Vessiot extension of those qsi fields $K$ for those $K\left[\sigma, \sigma^{-1}, \theta^{*}\right]$-modules $N$, which satisfy

(1) The $q s i$ base field $K$ contains $\left(C(t), \sigma, \sigma^{-1}, \theta^{*}\right)$,

(2) The operation of $\sigma$ and $\theta^{(1)}$ on the module $N$ as well as on the base field $K$, satisfy the relation

$$
\theta^{(1)}=\frac{1}{(q-1) t}\left(\sigma-\operatorname{Id}_{N}\right) .
$$

Note that the $C\left[\sigma, \sigma^{-1}, \theta^{*}\right]$-module $M$ given in (8.5), even after the base extension to any $K$ that satisfies (1) and (2), does not satisfy (2). Indeed, we see that in $K \otimes_{C} M$,

$$
\theta^{(1)}\left(1 \otimes m_{1}\right)=1 \otimes m_{2} \neq \frac{1}{t} \otimes m_{1}=\frac{1}{(q-1) t}\left(\sigma\left(1 \otimes m_{1}\right)-1 \otimes m_{1}\right) .
$$

\section{Quantum Picard-Vessiot ring of qsi module $M$ of (8.5)}

To determine the quantum Galois structure of the qsi field extension $L / C:=C(t) / C$, we analyzed the quantum normalization or the Galois hull of $L / C$ in Part I. We introduced the $q s i$ module $M:=C 1 \oplus C t$. The description of the Picard-Vessiot ring of the qsi module $M$ offers us another solution to the determination of the Galois group of the qsi field extension $L / C$. We recall that the universal Hopf morphism $\iota: L=C(t) \rightarrow F(\mathbb{Z}, C(t))[[X]]$ maps the element $t \in L$ to $\iota(t)=t Q+X$ so that $Q, X$ are in the Galois hull $\mathcal{L}$. Looking at Lemma 8.1, we are tempted to set

$$
R:=C\left\langle Q, Q^{-1}, X\right\rangle_{\text {alg }} \subset S:=C[[Q, X]]\left[Q^{-1}, X^{-1}\right],
$$


where we may consider $Q, X$ independent and non-commutative variables satisfying commutation relations

$$
\begin{gathered}
Q Q^{-1}=Q^{-1} Q=1, \quad X X^{-1}=X^{-1} X=1, \quad Q X=q X Q, \\
q X^{-1} Q^{-1}=q Q^{-1} X^{-1}, \quad X^{-1} Q=q Q X^{-1} .
\end{gathered}
$$

The ring $S$ is the set of formal Laurent series in $X$ and $Q$ with coefficients in $C$ so that we can add them as formal Laurent series. The product of two series in $S$ is well determined by the commutation relations (9.1). We must use $\langle\cdot, \cdot\rangle_{\text {alg }}$ instead of $[[\cdot, \cdot]]$. But we use the latter because it is simpler. The operator $\sigma, \sigma^{-1}, \theta^{(1)}$ operate on $C[[Q, X]]\left[Q^{-1}, X^{-1}\right]$ by

$$
\sigma^{ \pm 1}(X)=q^{ \pm 1} X, \quad \sigma^{ \pm 1}(Q)=q^{ \pm 1} Q, \quad \theta^{(0)}(X)=1, \quad \theta^{(0)}(Q)=0 .
$$

The rings $R$ and $S$ are qsi algebras.

We are going to show below, step by step, $R=C\left\langle Q, Q^{-1}, X\right\rangle$ is the Picard-Vessiot ring of the qsi module $M$. The definition and characterization of Picard-Vessiot ring is given during the explanation below.

Observation 9.1. - The left $C\left[\sigma, \sigma^{-1}, \theta^{(1)}\right]$-module $M$ has two solutions in the qsi ring $R$ linearly independent over $C$. In fact, setting

$$
Y:=\left[\begin{array}{cc}
Q & X \\
0 & 1
\end{array}\right] \in M_{2}(R)
$$

we have

$$
\sigma Y=\left[\begin{array}{ll}
q & 0 \\
0 & 1
\end{array}\right] Y \quad \text { and } \quad \theta^{(1)} Y=\left[\begin{array}{ll}
0 & 1 \\
0 & 0
\end{array}\right] Y .
$$

So the column vectors ${ }^{t}(Q, 0),{ }^{t}(X, 1) \in R^{2}$ are $C$-linearly independent solution of the system of equations (8.6).

The matrix $Y$ is an invertible element in the matrix algebra $M_{2}(R)$. We have

$$
\left[\begin{array}{cc}
Q & X \\
0 & 1
\end{array}\right]^{-1}=\left[\begin{array}{cc}
Q^{-1} & -Q^{-1} X \\
0 & 1
\end{array}\right] \in \mathrm{M}_{2}(R) .
$$

ObSeRvation 9.2. - The ring $R=C\left\langle X, Q, Q^{-1}\right\rangle_{\text {alg }}$ has no zero-divisors. We can consider the ring $K$ of total fractions of $R=C\left\langle X, Q, Q^{-1}\right\rangle_{\text {alg }}$.

Proof. - Every non-zero element is invertible. In other words, the ring $S$ is a skew field. So we can consider the smallest skew field containing the image of $R$ in $S$.

ObSeRvation 9.3. - Let $K$ be the ring of total fractions of $R$. The ring of qsi constants $C_{K}$ coincides with $C$. The ring of $\theta^{*}$ constants of $C[X, Q]]\left[X^{-1}, Q^{-1}\right]$ is $C(Q)$. Moreover as we assume that $q$ is not a root of unity, the ring of $\sigma$-constants of $C(Q)$ is equal to $C$. 
Lemma 9.4. - The non-commutative qsi algebra $R$ is simple, that is, there is no qsi bilateral ideal of $R$ except for the zero-ideal and $R$.

Proof. - Let $I$ be a non-zero qsi bilateral ideal of $R$. We take an element

$$
0 \neq f:=a_{0}+X a_{1}+\cdots+X^{n} a_{n} \in I,
$$

where $a_{i} \in C\left[Q, Q^{-1}\right]$ for $0 \leqslant i \leqslant n$. We may assume $a_{n} \neq 0$. Applying $\theta^{(n)}$ to the element $f$, we conclude that $0 \neq a_{n} \in C\left[Q, Q^{-1}\right]$ is in the ideal $I$. Multiplying a monomial $b Q^{l}$ with $b \in C$, we find a polynomial $h=1+b_{1} Q+\cdots+b_{s} Q^{s} \in C[Q]$ with $b_{s} \neq 0$ in the ideal $I$. We show that 1 is in $I$ by induction on $s$. If $s=0$, then there is nothing to prove. Assume that the assertion is proved for $s \leqslant m$. We have to show the assertion for $s=m+1$. Then, since $Q^{i}$ is an eigenvector of the operator $\sigma$ with eigenvalue $q^{i}$ for $i \in \mathbb{N}$,

$$
\frac{1}{q^{m+1}-1}\left(q^{m+1} h-\sigma(h)\right)=1+c_{1} Q+\cdots+c_{m} Q^{m} \in C[Q]
$$

is an element of $I$ and by induction hypothesis 1 is in the ideal $I$.

ObServation 9.5. - The extension $R / C$ trivializes the $C\left[\sigma, \sigma^{-1}, \theta^{*}\right]$ module $M$. Namely, there exist qsi constants $c_{1}, c_{2} \in R \otimes_{C} M$ such that there exists a left $R$-qsi module isomorphism

$$
R \otimes_{C} M \simeq R c_{1} \oplus R c_{2} .
$$

Proof. - In fact, it is sufficient to set

$$
c_{1}:=Q^{-1} m_{1}-Q^{-1} X m_{2}, \quad c_{2}:=m_{2} .
$$

Then

$$
\sigma\left(c_{1}\right)=c_{1}, \quad \sigma\left(c_{2}\right)=c_{2}, \quad \theta^{(1)}\left(c_{2}\right)=0
$$

and

$$
\theta^{(1)}\left(c_{1}\right)=q^{-1} Q^{-1} \theta^{(1)}\left(m_{1}\right)-q^{-1} Q^{-1} m_{2}=q^{-1} Q^{-1} m_{2}-q^{-1} Q^{-1} m_{2}=0 .
$$

So we have an $\left(R, \sigma, \sigma^{-1}, \theta^{*}\right)$-module isomorphism $R \otimes_{C} M \simeq R c_{1} \oplus R c_{2}$.

Observation 9.6. - The Hopf algebra $\mathfrak{H}_{q}=C\left\langle u, u^{-1}, v\right\rangle$ with $u v=$ qvu co-acts from right on the non-commutative algebra $R$. Namely, we have an algebra morphism

$$
R \longrightarrow R \otimes_{C} \mathfrak{H}_{q}
$$

sending

$$
X \longmapsto X \otimes 1+Q \otimes v, \quad Q \longmapsto Q \otimes u, \quad Q^{-1} \longmapsto Q^{-1} \otimes u^{-1} .
$$

Morphism (9.4) is compatible with $C\left[\sigma, \sigma^{-1}, \theta^{(1)}\right]$-module structures, where $\sigma, \sigma^{-1}$ and $\theta^{(1)}$ operate on the Hopf algebra $\mathfrak{H}_{q}$ trivially. 
We can prove the assertion of Observation 9.6 by a simple direct calculation, which is very much unsatisfactory. For, we are eager to know where the Hopf algebra $\mathfrak{H}_{q}$ comes from. We answer this question in two steps:

(1) Characterization of the non-commutative algebra $\mathfrak{H}_{q}$.

(2) Origin of the co-multiplication structure on the Hopf algebra $\mathfrak{H}_{q}$.

We answer question (1) in Corollary 9.9, and question (2) in Observation 9.12. To this end, we admit the algebra structure of $\mathfrak{H}_{q}$ and characterize it.

Let us first fix some notations. For a not necessarily commutative $C$-qsi algebra $T$ and for a morphism $\varphi: R \rightarrow T$ of $q s i$ algebras over $C$, we set

$$
\varphi(Y)=\left[\begin{array}{cc}
\varphi(Q) & \varphi(t) \\
0 & 1
\end{array}\right] .
$$

Then $\varphi(Y)$ is an invertible element in the matrix ring $M_{2}(T)$, with inverse being given by

$$
\varphi(Y)^{-1}=\left[\begin{array}{cc}
\varphi(Q)^{-1} & -\varphi(Q)^{-1} \varphi(t) \\
0 & 1
\end{array}\right] .
$$

We have seen above the following Lemma.

LEMma 9.7. - For a not necessarily commutative $C$-qsi algebra $T$ and for elements $a, b \in T$, there exists a $C$-qsi algebra morphism $\varphi: R \rightarrow T$ such that $\varphi(Q)=a$ and $\varphi(t)=b$ if and only if the following three conditions are satisfied:

(1) We have a commutation relation

$$
a b=q b a,
$$

(2) the elements a, $b$ satisfies difference differential equations

$$
\sigma(a)=q a, \quad \theta^{(1)}(a)=0, \quad \sigma(b)=q b, \quad \theta^{(1)}(b)=1,
$$

(3) the element a is invertible in the ring $T$ or equivalently the matrix

$$
\left[\begin{array}{ll}
a & b \\
0 & 1
\end{array}\right]
$$

is invertible in the ring $M_{2}(T)$.

Corollary 9.8. - Let $\varphi: R \rightarrow T$ be a qsi algebra morphism over $C$. Using the notation above, let

$$
H^{\prime}=\left[\begin{array}{cc}
u^{\prime} & v^{\prime} \\
0 & 1
\end{array}\right] \in M_{2}\left(C_{T}\right)
$$

be an invertible element in the matrix ring $M_{2}\left(C_{T}\right)$ satisfying the following two conditions. 
(1) $u^{\prime} v^{\prime}=q v^{\prime} u^{\prime}$ and the element $u^{\prime}$ is invertible in the ring $C_{T}$ of constants of $T$.

(2) The set $\left\{u^{\prime}, v^{\prime}\right\}$ and the set of entries of the matrix $\varphi(Y)$ are elementwisely commutative.

Then, there exists a qsi algebra morphism $\psi \in \operatorname{Hom}_{C-q s i}(R, T)$ over $C$ such that

$$
\psi(Y)=\varphi(Y) H^{\prime} .
$$

Proof. - By Lemma 9.7, the matrix

$$
\varphi(Y)=\left[\begin{array}{ll}
a & b \\
0 & 1
\end{array}\right]
$$

satisfies conditions of Lemma 9.7. This, together with the assumption (1) and (2) in this Corollary, implies that the matrix

$$
\varphi(Y) H^{\prime}
$$

satisfies the conditions of Lemma 9.7. Now the assertion follows from Lemma 9.7.

In particular if we take $T=R \otimes_{C} \mathfrak{H}_{q}$ and

$$
H^{\prime}=\left[\begin{array}{ll}
u & v \\
0 & 1
\end{array}\right] \in \mathrm{M}_{2}\left(\mathfrak{H}_{q}\right)
$$

and

$$
\varphi_{0}(Y)=Y=\left[\begin{array}{ll}
Q & t \\
0 & 1
\end{array}\right]=\left[\begin{array}{cc}
Q \otimes 1 & t \otimes 1 \\
0 & 1
\end{array}\right],
$$

then the conditions of Corollary are satisfied and we get the morphism $R \rightarrow$ $R \otimes_{C} \mathfrak{H}_{q}$ in the Observation 9.6.

It also characterizes the algebra $\mathfrak{H}_{q}$. We notice that an $S \in \mathrm{ob}(\mathrm{NCAlg} / C)$ has a trivial $q s i$-algebra structure over $C$, if we set $\sigma=\operatorname{Id}_{S}, \theta^{(0)}=\operatorname{Id}_{S}$ and $\theta^{(i)}=0$ for $i \geqslant 1$. Namely, if we consider a functor

$$
F:(\text { NCAlg } / C) \longrightarrow \text { (Set) }
$$

on the category of not necessarily commutative $C$-algebras defined by

$$
F(S)=\operatorname{Hom}_{C-q s i}\left(R, R \otimes_{C} S\right) \text { for } S \in \mathrm{ob}(\operatorname{NCAlg} / C),
$$

then the functor $F$ is representable by the algebra $\mathfrak{H}_{q}$. have

COROllary 9.9. - For an object $S$ of the category $(\mathrm{NCAlg} / C)$, we

$$
\operatorname{Hom}_{C-q s i}\left(R, R \otimes_{C} S\right) \simeq \operatorname{Hom}_{C-a l g}\left(\mathfrak{H}_{q}, S\right),
$$

where the left-hand side denotes the set of qsi algebra morphisms over $C$ and the right-hand side is the set of $C$-algebra morphisms. 
Proof. - If we notice $C_{R \otimes_{C} S}=S$ and take as $\varphi: R \rightarrow R \otimes_{C} S$ the canonical inclusion

$$
\varphi_{0}: R \longrightarrow R \otimes_{C} S, \quad a \longmapsto a \otimes 1,
$$

it follows from Corollary 9.8 that we have a map

$$
\operatorname{Hom}_{C-a l g}\left(\mathfrak{H}_{q}, S\right) \longrightarrow \operatorname{Hom}_{C-q s i}\left(R, R \otimes_{C} S\right) .
$$

that sends $\pi \in \operatorname{Hom}_{C \text {-alg }}\left(\mathfrak{H}_{q}, S\right)$ to $\psi \in \operatorname{Hom}_{C-q s i}\left(R, R \otimes_{C} S\right)$ such that

$$
\psi(Y)=\varphi_{0}(Y)\left[\begin{array}{cc}
\pi(u) & \pi(v) \\
0 & 1
\end{array}\right] .
$$

To get the mapping of the other direction, let $\psi: R \rightarrow R \otimes_{C} S$ be a qsi morphism over $C$. Note that $S=C_{R} \otimes_{C} S$. Then using the morphism $\varphi_{0}$ above, since both $\varphi_{0}(Y)$ and $\psi(Y)$ are solutions to the linear qsi equations (8.6), an easy calculation shows that the entries of the matrix

$$
H^{\prime}:=\varphi(Y)^{-1} \psi(Y) \in M_{2}\left(R \otimes_{C} S\right)
$$

has entries in $S$, and is of the form

$$
H^{\prime}=\left[\begin{array}{cc}
u^{\prime} & v^{\prime} \\
0 & 1
\end{array}\right] .
$$

We single out a Sub-lemma because we later use the same argument.

SuB-LEMmA 9.10. — We have the commutation relation

$$
u^{\prime} v^{\prime}=q v^{\prime} u^{\prime} .
$$

Proof of Sub-lemma. - Let us set

$$
\varphi_{0}(Y)=\left[\begin{array}{ll}
a & b \\
0 & 1
\end{array}\right], \quad \text { and } \quad \psi(Y)=\left[\begin{array}{cc}
a^{\prime} & b^{\prime} \\
0 & 1
\end{array}\right]
$$

So we have

$$
\begin{aligned}
a b & =q b a & a^{\prime} b^{\prime} & =q b^{\prime} a^{\prime} \\
a^{\prime} & =a u^{\prime} & b^{\prime} & =a v^{\prime}+b .
\end{aligned}
$$

Since the set $\left\{u^{\prime}, v^{\prime}\right\} \subset S$ and $\{a, b\} \subset R$ are element-wisely commutative in $R \otimes_{C} S$, substituting equations (9.7) into the second equation of (9.6) and then using the first equation of (9.6), Sublemma follows.

By Sub-lemma, we get a morphism $\pi_{\psi}: \mathfrak{H}_{q} \rightarrow S$ sending $u$ to $u^{\prime}$ and $v$ to $v^{\prime}$. So $\psi \mapsto \pi_{\psi}$ gives the mapping of the other direction.

Remark 9.11. - For two qsi morphisms $\varphi, \psi: R \rightarrow T$ over $C$, let us set

$$
\varphi(Y)=\left[\begin{array}{ll}
a & b \\
0 & 1
\end{array}\right], \quad \psi(Y)=\left[\begin{array}{ll}
c & d \\
0 & 1
\end{array}\right] .
$$


It follows from difference differential equations

$$
\begin{array}{ll}
\sigma\left(\left[\begin{array}{ll}
a & b \\
0 & 1
\end{array}\right]\right)=\left[\begin{array}{ll}
q & 0 \\
0 & 1
\end{array}\right]\left[\begin{array}{ll}
a & b \\
0 & 1
\end{array}\right], & \theta^{(1)}\left(\left[\begin{array}{ll}
a & b \\
0 & 1
\end{array}\right]\right)=\left[\begin{array}{ll}
0 & 1 \\
0 & 0
\end{array}\right]\left[\begin{array}{ll}
a & b \\
0 & 1
\end{array}\right], \\
\sigma\left(\left[\begin{array}{ll}
c & d \\
0 & 1
\end{array}\right]\right)=\left[\begin{array}{ll}
q & 0 \\
0 & 1
\end{array}\right]\left[\begin{array}{ll}
c & d \\
0 & 1
\end{array}\right], & \theta^{(1)}\left(\left[\begin{array}{ll}
c & d \\
0 & 1
\end{array}\right]\right)=\left[\begin{array}{ll}
0 & 1 \\
0 & 0
\end{array}\right]\left[\begin{array}{ll}
c & d \\
0 & 1
\end{array}\right]
\end{array}
$$

that the entries of the matrix

$$
\left[\begin{array}{cc}
u^{\prime} & v^{\prime} \\
0 & 1
\end{array}\right]:=\varphi(Y)^{-1} \psi(Y)=\left[\begin{array}{ll}
a & b \\
0 & 1
\end{array}\right]^{-1}\left[\begin{array}{cc}
c & d \\
0 & 1
\end{array}\right]=\left[\begin{array}{cc}
a^{-1} c & a^{-1} d-a^{-1} b \\
0 & 1
\end{array}\right]
$$

are constants. Namely,

So equivalently

$$
\left[\begin{array}{cc}
u^{\prime} & v^{\prime} \\
0 & 1
\end{array}\right] \in M_{2}\left(C_{T}\right)
$$

$$
\psi(Y)=\varphi(Y)\left[\begin{array}{cc}
u^{\prime} & v^{\prime} \\
0 & 1
\end{array}\right]
$$

The entries of the matrix do not necessarily satisfy the commutation relation $u^{\prime} v^{\prime}=q u^{\prime} v^{\prime}$.

For algebras $A, S \in \mathrm{ob}(\mathrm{NCAlg} / C)$, we set

$$
A(S):=\operatorname{Hom}_{C \text {-alg }}(A, S)
$$

that is the set of $C$-algebra morphisms.

OBSERVATION 9.12 (Origin of co-multiplication of the Hopf algebra $\mathfrak{H}_{q}$ ). The co-multiplication $\Delta: \mathfrak{H}_{q} \rightarrow \mathfrak{H}_{q} \otimes_{C} \mathfrak{H}_{q}$ comes from the multiplications of matrices. More precisely, to construct an algebra morphism $\Delta: \mathfrak{H}_{q} \rightarrow$ $\mathfrak{H}_{q} \otimes_{C} \mathfrak{H}_{q}$, it is sufficient to give a functorial morphism

$$
\mathfrak{H}_{q} \otimes_{C} \mathfrak{H}_{q}(S) \longrightarrow \mathfrak{H}_{q}(S) \quad \text { for } S \in \mathrm{ob}(\mathrm{NCAlg} / C) \text {. }
$$

An element of $\mathfrak{H}_{q} \otimes_{C} \mathfrak{H}_{q}(S)$ being given, it determines a pair $\left(\pi_{1}, \pi_{2}\right)$ of morphisms $\pi_{1}, \pi_{2}: \mathfrak{H}_{q} \rightarrow S$ such that the images $\pi_{1}\left(\mathfrak{H}_{q}\right)$ and $\pi_{2}\left(\mathfrak{H}_{q}\right)$ are element-wisely commutative. This condition is equivalent to element-wisely commutativity of the set of the entries $\left\{u_{1}^{\prime}, v_{1}^{\prime}\right\}$ and $\left\{u_{2}^{\prime}, v_{2}^{\prime}\right\}$ of the matrices $H_{1}^{\prime}:=\left[\begin{array}{cc}u_{1}^{\prime} & v_{1}^{\prime} \\ 0 & 1\end{array}\right]:=\left[\begin{array}{cc}\pi_{1}(u) & \pi_{1}(v) \\ 0 & 1\end{array}\right], \quad H_{2}^{\prime}:=\left[\begin{array}{cc}u_{2}^{\prime} & v_{2}^{\prime} \\ 0 & 1\end{array}\right]:=\left[\begin{array}{cc}\pi_{2}(u) & \pi_{2}(v) \\ 0 & 1\end{array}\right]$.

We show that there exists a morphism $\pi_{3}: \mathfrak{H}_{q} \rightarrow S$ such that

$$
H_{1}^{\prime} H_{2}^{\prime}=\left[\begin{array}{cc}
\pi_{3}(u) & \pi_{3}(v) \\
0 & 1
\end{array}\right]
$$

In fact, by Corollary 9.8, there exists a morphism $\psi_{1}: R \rightarrow R \otimes_{C} S$ such that

$$
\begin{gathered}
\psi_{1}(Y)=\varphi_{0}(Y) H_{1}^{\prime} . \\
-1402-
\end{gathered}
$$


Then since the entries of $H_{2}^{\prime}$ and the union

$$
\text { (the entries of } \left.\varphi_{0}(Y)\right) \cup\left(\text { the entries of } H_{1}^{\prime}\right)
$$

are element-wisely commutative and consequently the entries of $H_{2}^{\prime}$ and the entries of the product $\varphi_{0}(Y) H_{1}^{\prime}$ are element-wisely commutative, by Corollary 9.8 , there exists a morphism $\psi_{2}: R \rightarrow R \otimes_{C} S$ such that

$$
\psi_{2}(Y)=\left(\varphi_{0}(Y) H_{1}^{\prime}\right) H_{2}^{\prime}=\varphi_{0}(Y)\left(H_{1}^{\prime} H_{2}^{\prime}\right) .
$$

So if we note that the entries of $H_{1}^{\prime} H_{2}^{\prime}$ and the entries of the matrix $\varphi_{0}(Y)$ are element-wisely commutative, writing

$$
H_{1}^{\prime} H_{2}^{\prime}=\left[\begin{array}{cc}
u_{3}^{\prime} & v_{3}^{\prime} \\
0 & 1
\end{array}\right],
$$

the argument of the proof of Sub-lemma 9.10 shows us that, we have $u_{3}^{\prime} v_{3}^{\prime}=$ $q v_{3}^{\prime} u_{3}^{\prime}$. Hence there exists a morphism $\pi_{3}: \mathfrak{H}_{q} \rightarrow S$ sending $u$ to $u_{3}^{\prime}$ and $v$ to $v_{3}^{\prime}$. Now the mapping $\left(\pi_{1}, \pi_{2}\right) \mapsto \pi_{3}$ defines the morphism (9.8).

Proposition 9.13. - The right co-action

$$
\rho: R \longrightarrow R \otimes_{C} \mathfrak{H}_{q}
$$

is an $\mathfrak{H}_{q}$ torsor in the following sense. The $C$-algebra morphism $\rho$ defines a C-linear map

$$
\varphi: R \otimes_{C} R \longrightarrow R \otimes_{C} \mathfrak{H}_{q}
$$

such that $\varphi(a b)=(a \otimes 1) \rho(b)$ for $a, b \in R$. The C-linear map $\varphi$ is an isomorphism of the $C$-vector spaces.

Proof. - The $C$-linear morphism $\varphi$ is, in fact, a left $R=\left(R \otimes_{C} 1\right)$-module morphism. The $C$-linear algebra morphism $\rho: R \rightarrow R \otimes_{C} \mathfrak{H}_{q}$ sends $C$-linear basis $\left\{Q^{m} X^{n}\right\}_{m \in \mathbb{Z}, n \in \mathbb{N}}$ of $R$ to

$$
\begin{aligned}
\rho\left(Q^{m} X^{n}\right)= & \varphi\left(1 \otimes Q^{m} X^{n}\right) \\
= & (Q \otimes u)^{m}(X \otimes 1+Q \otimes v)^{n} \\
= & \left(Q^{m+n} \otimes 1\right)\left(1 \otimes u^{m}\right) \\
& \times\left(1 \otimes v^{n}+\text { an } R \text {-linear combination of } 1 \otimes v^{i} \text { for } 0 \leqslant i \leqslant n-1\right) .
\end{aligned}
$$

Since $Q$ is an invertible element of $R$, the last elements above form an $R=$ $\left(R \otimes_{C} 1\right)$-linear basis of $R \otimes_{C} \mathfrak{H}_{q}$, to which the $R=\left(R \otimes_{C} 1\right)$ linear basis $\{1 \otimes$ $\left.Q^{m} X^{n}\right\}_{m \in \mathbb{Z}, n \in \mathbb{N}}$ is sent by the $R$-linear map $\varphi: R \otimes_{C} R \rightarrow R \otimes_{C} \mathfrak{H}_{q}$. So the $R=(R \otimes 1)$-module morphism $\varphi: R \otimes_{C} R \rightarrow R \otimes_{C} \mathfrak{H}_{q}$ is an isomorphism. 


\section{On the uniqueness of the Picard-Vessiot ring}

We show that our Picard-Vessiot ring $R / C$ is unique.

Given a not necessarily commutative algebra $\mathcal{R}$ and a positive integer $n$, we let $\mathcal{R}^{n}$ (resp., ${ }^{t} \mathcal{R}^{n}$ ) denote the right or left $\mathcal{R}$-module of column (resp., row) vectors with $n$ entries in $\mathcal{R}$.

Lemma 10.1. - Suppose that an $n \times n$-matrix $Y \in M_{n}(\mathcal{R})$ consists of $n$ column vectors $\mathbf{y}_{1}, \ldots, \mathbf{y}_{n}$ and of $n$ row vectors ${ }^{t} \mathbf{w}_{1}, \ldots,{ }^{t} \mathbf{w}_{n}$, so that

$$
Y=\left(\mathbf{y}_{1}, \ldots, \mathbf{y}_{n}\right)={ }^{t}\left(\mathbf{w}_{1}, \ldots, \mathbf{w}_{n}\right) .
$$

Then the following are equivalent:

(1) $Y$ is invertible $M_{n}(\mathcal{R})$;

(2) $\mathbf{y}_{1}, \ldots, \mathbf{y}_{n}$ form a frees basis of the right $\mathcal{R}$-module $\mathcal{R}^{n}$;

(3) ${ }^{t} \mathbf{w}_{1}, \ldots,{ }^{t} \mathbf{w}_{n}$ form a frees basis of the left $\mathcal{R}$-module ${ }^{t} \mathcal{R}^{n}$.

Proof. - The equivalence (1) $\Leftrightarrow(2)$ (resp., (1) $\Leftrightarrow(3)$ ) follows from the canonical algebra-isomorphism from $M_{n}(\mathcal{R})$ to the algebra of endomorphisms of the right $\mathcal{R}$-module $\mathcal{R}^{n}$ (resp., the left $\mathcal{R}$-module ${ }^{t} \mathcal{R}^{n}$ ).

Let $M$ be a left $C\left[\sigma, \sigma^{-1}, \theta^{*}\right]$-module that is of finite dimension $n$ as a $C$-vector space. Let $\left\{m_{1}, m_{2}, \ldots, m_{n}\right\}$ be a basis of the $C$-vector space $M$. Setting $m={ }^{t}\left(m_{1}, m_{2}, \ldots, m_{n}\right)$, there exist matrices $A \in \mathrm{GL}_{n}(C), B \in$ $M_{n}(C)$ satisfying

$$
\sigma(m)=A m, \quad \theta^{(1)}(m)=B m .
$$

As we have seen in Section 9 , the left $C\left[\sigma, \sigma^{-1}, \theta^{*}\right]$-module $M$ defines a system of qsi equation

$$
\sigma(y)=A y, \quad \theta^{(1)}(y)=B y
$$

where $y$ is an unknown column vector with $n$ entries. We are interested in solutions $y \in \mathcal{R}^{n}$ for a qsi algebra $\mathcal{R}$ over $C$.

Definition 10.2. - For qsi algebra $\mathcal{R}$ over $C$, we say that a set $\left\{\mathbf{y}_{1}, \mathbf{y}_{2}, \ldots, \mathbf{y}_{n}\right\}$ of solutions to (10.2) so that $\mathbf{y}_{i} \in \mathcal{R}^{n}$ for $1 \leqslant i \leqslant n$, is a fundamental system of solutions to (10.2) if the matrix $Y=\left(\mathbf{y}_{1}, \mathbf{y}_{2}, \ldots, \mathbf{y}_{n}\right) \in$ $M_{n}(R)$ satisfies the equivalent conditions (1), (2) of Lemma 10.1.

The dual to a fundamental system is a trivializing matrix of the qsi module $M$.

Definition 10.3. - We assume that the qsi module $\mathcal{R} \otimes_{C} M$ is trivialized over a $C$-qsi ring $\mathcal{R}$. Namely, there exist elements

$$
\begin{gathered}
c_{1}, c_{2}, \ldots, c_{n} \in \mathcal{R} \otimes_{C} M \\
-1404-
\end{gathered}
$$


such that

$$
\sigma\left(c_{i}\right)=c_{i}, \quad \theta^{(1)}\left(c_{i}\right)=0 \text { for every } 1 \leqslant i \leqslant n
$$

and such that we have $\mathcal{R}$-module decomposition

$$
\mathcal{R} \otimes_{C} M=\bigoplus_{i=1}^{n} \mathcal{R} c_{i} .
$$

So writing the elements $c_{i}$ 's as a left $\mathcal{R}$-linear combination of the basis

$$
\left\{m_{1}, m_{2}, \ldots, m_{n}\right\},
$$

we get a matrix $Z \in M_{n}(\mathcal{R})$ such that

$$
{ }^{t}\left(c_{1}, c_{2}, \ldots, c_{n}\right)=Z^{t}\left(m_{1}, m_{2}, \ldots, m_{n}\right) .
$$

We call the matrix $Z \in M_{n}(\mathcal{R})$ a trivializing matrix of qsi module $M$ over $\mathcal{R}$.

LEMMA 10.4. - A trivializing matrix over $\mathcal{R}$ is invertible in the matrix $\operatorname{ring} M_{n}(\mathcal{R})$.

Proof. - This follows by the same argument of proving Lemma 10.1.

Now we make clear the relation between fundamental system and trivializing matrix.

Proposition 10.5. - The following four conditions on an invertible matrix $Y \in M_{n}(\mathcal{R})$ are equivalent. We denote $Y^{-1}$ by $Z$ or $Y=Z^{-1}$.

(1) The matrix $Y$ satisfies qsi equations

$$
\sigma(Y)=A Y, \quad \theta^{(1)}(Y)=B Y,
$$

with $A, B$ being the matrices in (10.1).

(2) The matrix $Y$ is a fundamental system of solutions of $M$.

(3) The matrix $Z$ satisfies qsi equations

$$
\sigma(Z)=Z A^{-1}, \quad \theta^{(1)}(Z)=-Z A^{-1} B
$$

(4) The matrix $Z$ is a trivializing matrix for $M$ over $\mathcal{R}$.

Proof. - The equivalence of conditions (1) and (2) follows from Lemma 10.1 and Definition 10.2. To prove the equivalence of (3) and (4), we set

$$
{ }^{t}\left(c_{1}, c_{2}, \ldots, c_{n}\right):=Z^{t}\left(m_{1}, m_{2}, \ldots, m_{n}\right),
$$

where the $m_{i}$ 's are the basis of $M$ chosen above, so that

$$
c_{i}=\sum_{l=1}^{n} z_{i l} m_{l} \text { for every } 1 \leqslant i \leqslant n .
$$

It is convenient to introduce

$$
\mathbf{c}:={ }^{t}\left(c_{1}, c_{2}, \ldots, c_{n}\right), \quad \text { and } \quad \mathbf{m}:={ }^{t}\left(m_{1}, m_{2}, \ldots, m_{n}\right) .
$$


So we have

$$
\mathbf{c}=Z \mathbf{m} \text {. }
$$

Now we assume Condition (3) and show Condition (4). To this end, we prove that the $c_{i}$ 's that are elements of $\mathcal{R} \otimes_{C} M$, are constants. In fact, if we apply $\sigma$ to $(10.5)$, it follows from the first equation in (10.3),

$$
\begin{aligned}
\sigma(\mathbf{c}) & =\sigma(Z) \sigma(\mathbf{m}) \\
& =\left(Z A^{-1}\right)(A \mathbf{m}) \\
& =Z \mathbf{m} \\
& =\mathbf{c} .
\end{aligned}
$$

Namely $\sigma(\mathbf{c})=\mathbf{c}$. Now we apply $\theta^{(1)}$ to $(10.5)$ to get

$$
\begin{aligned}
\theta^{(1)}(\mathbf{c}) & =\theta^{(1)}(Z) \mathbf{m}+\sigma(Z) \theta^{(1)}(\mathbf{m}) \\
& =\left(-Z A^{-1} B\right) \mathbf{m}+\left(Z A^{-1}\right) B \mathbf{m} \\
& =0 .
\end{aligned}
$$

So $\theta^{(1)}(\mathbf{c})=0$ and $\mathbf{c}$ is a constant. Hence $Z$ is a trivializing matrix by Definition 10.3 and the argument of proving Lemma 10.1. Conversely, we start from Condition (4). If we recall

$$
\mathbf{c}:=Z \mathbf{m}
$$

then, as we assume Condition (4), $\mathbf{c}$ is a constant. Applying $\sigma$ and $\theta^{(1)}$ to (10.6), we get Condition (3).

It remains to show the equivalence of (1) and (3). Let us assume (1) and show (3). If we apply the automorphism $\sigma$ to the equality $Z Y=\mathrm{I}_{n}$, the first equality in (10.3) implies the first equality of (10.4). On the other hand, applying $\theta^{(1)}$ to the equality $Z Y=I_{n}$, we get

$$
\theta^{(1)}(Z) Y+\sigma(Z) \theta^{(1)}(Y)=0 .
$$

It follows from equation (10.3)

$$
\theta^{(1)}(Z) Y+Z A^{-1} B Y=0 .
$$

Since the matrix $Y$ is invertible, we conclude

$$
\theta^{(1)} Z=-Z A^{-1} B \text {. }
$$

So the matrix $Z$ satisfies Condition (3). The proof of the converse that Condition (3) implies (1) is similar. Applying first $\sigma$ and then $\theta^{(1)}$ to $Z Y=$ $I_{n}$, we immediately get Condition (1).

We are ready to characterize the Picard-Vessiot ring $R / C$. Besides the properties we mentioned above, we have a $C$-morphism or a $C$-valued point of the abstract ring $R^{\natural}$

$$
R^{\natural} \longrightarrow C \text { sending } Q^{ \pm 1} \longmapsto 1, \quad X \longmapsto 0 .
$$


We sometimes call it a $C$-rational point.

LEMMA 10.6. - Let $\mathcal{R}$ be a simple qsi algebra over $C$. If the abstract algebra $\mathcal{R}^{\natural}$ has a $C$-valued point, then the ring of constants of $\mathcal{R}$ coincides with $C$.

Proof. - Assume to the contrary that there is a constant $f$ in $\mathcal{R} \backslash C$. Let

$$
\varphi: \mathcal{R} \longrightarrow C
$$

be a $C$-valued point. We set $c:=\varphi(f)$ that is an element of $C$. So the element $f-c \neq 0$ is a constant of qsi algebra $\mathcal{R}$. Therefore the bilateral ideal $I$ generated by $f-c$ is a qsi bilateral ideal of $\mathcal{R}$ because the ideal $I$ is generated by the constant $f-c$. As the ideal $I$ contains $f-c \neq 0$, the simplicity of $\mathcal{R}$ implies $I=\mathcal{R}$. So there would be a positive integer $n$ and elements $a_{i}, b_{i} \in \mathcal{R}$ for $0 \leqslant i \leqslant n$ such that

$$
\sum_{i=1}^{n} a_{i}(f-c) b_{i}=1 .
$$

Applying the morphism $\varphi$, we would have $0=1$ in $C$ by $(10.9)$ that is a contradiction.

So far in this Section, we studied general $C\left[\sigma, \sigma^{-1}, \theta^{*}\right]$-module $M$. From now on, we come back to the $C\left[\sigma, \sigma^{-1}, \theta^{*}\right]$-module $M$ in Section 9 so that writing $\mathbf{m}={ }^{t}\left(m_{1}, m_{2}\right)$,

$$
\begin{gathered}
M=C m_{1} \oplus C m_{2}, \\
\sigma(\mathbf{m})=A \mathbf{m}, \quad \theta^{(1)}(\mathbf{m})=B \mathbf{m},
\end{gathered}
$$

where

$$
A=\left[\begin{array}{ll}
q & 0 \\
0 & 1
\end{array}\right] \quad \text { and } \quad B=\left[\begin{array}{ll}
0 & 1 \\
0 & 0
\end{array}\right]
$$

THEOREM 10.7. - Using the notation above, we can characterize the Picard-Vessiot ring $R / C$ for $M$ constructed in Section 9, in the following way.

Let $\mathcal{R} / C$ be a qsi extension satisfying the following conditions.

(1) There exists a fundamental system of solutions $\mathbf{Y} \in M_{2}(\mathcal{R})$ for $M$ such that

$$
\mathcal{R}=C\left\langle\mathbf{Y}, \mathbf{Y}^{-1}\right\rangle_{\text {alg }}
$$

(2) The qsi algebra $\mathcal{R}$ is simple.

(3) There exists a $C$-rational point of the abstract $C$-algebra $\mathcal{R}^{\natural}$.

Then the qsi algebra $\mathcal{R}$ is $C$-isomorphic to the Picard-Vessiot ring $R$. 
Proof. - Let us express $\mathbf{Y}$ in the matrix form:

$$
\mathbf{Y}=\left[\begin{array}{ll}
a & b \\
c & d
\end{array}\right] \in M_{2}(\mathcal{R})
$$

Hence by (10.11), the matrix $\mathbf{Y}$ satisfies

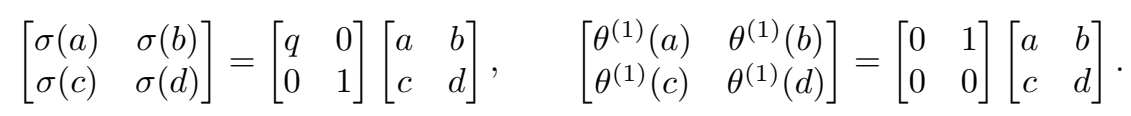

or to be more concrete

$$
\begin{array}{llll}
\sigma(a)=q a, & \theta^{(1)}(a)=c, & \sigma(c)=c, & \theta^{(1)}(c)=0, \\
\sigma(b)=q b, & \theta^{(1)}(b)=d, & \sigma(d)=d, & \theta^{(1)}(d)=0 .
\end{array}
$$

It follows from (10.12) and (10.13) that $c, d$ are constants of $\mathcal{R}$. By Lemma 10.6 and assumption (2) on $\mathcal{R}$, the ring $C_{\mathcal{R}}$ of constants of $\mathcal{R}$ coincides with $C$. So $c, d$ are complex numbers and hence by replacing the column vectors of the matrix $\mathbf{Y}$ by their appropriate $C$-linear combinations if necessary, we may assume that $c=0$ and $d=1$ so that

$$
\mathbf{Y}=\left[\begin{array}{ll}
a & b \\
0 & 1
\end{array}\right] \text {. }
$$

Consequently the set of equations (10.12) and (10.13) reduces to

$$
\sigma(a)=q a, \quad \theta^{(1)}(a)=0, \quad \sigma(b)=q b, \quad \theta^{(1)}(b)=1 .
$$

Since the matrix $\mathbf{Y}$ is invertible, $a$ is an invertible element of the ring $\mathcal{R}$. We show that $f:=q a^{-1} b-b a^{-1} \in \mathcal{R}$ is a constant. In fact, since $q$ is in the center of $\mathcal{R}$, it follows from (10.14) that

$$
\begin{aligned}
\sigma(f) & =q \sigma\left(a^{-1}\right) \sigma(b)-\sigma(b) \sigma\left(a^{-1}\right) \\
& =q a^{-1} q^{-1} q b-q b a^{-1} q^{-1} \\
& =q a^{-1} b-b a^{-1} \\
& =f
\end{aligned}
$$

and

$$
\begin{aligned}
\theta^{(1)}(f) & =q \sigma\left(a^{-1}\right) \theta^{(1)}(b)-\theta^{(1)}(b) a^{-1} \\
& =q a^{-1} q^{-1} 1-1 a^{-1} \\
& =0 .
\end{aligned}
$$

Therefore $f$ is a constant. Now we denote by $g$ the constant

$$
\frac{f}{1-q} \text {. }
$$

and set

$$
\begin{gathered}
b^{\prime}:=b+g a . \\
-1408-
\end{gathered}
$$


Then

$$
\mathbf{Y}^{\prime}:=\left[\begin{array}{ll}
a & b^{\prime} \\
0 & 1
\end{array}\right]
$$

is a fundamental system of solutions so that

$$
\sigma(a)=q a, \quad \sigma\left(a^{-1}\right)=q^{-1} a^{-1}, \quad \sigma\left(b^{\prime}\right)=q b^{\prime}, \quad \theta^{(1)}\left(b^{\prime}\right)=1 .
$$

Moreover, we have

$$
\mathcal{R}=C\left\langle\mathbf{Y}, \mathbf{Y}^{-1}\right\rangle_{\mathrm{alg}}=C\left\langle\mathbf{Y}^{\prime}, \mathbf{Y}^{\prime-1}\right\rangle_{\mathrm{alg}}=C\left\langle a, b^{\prime}, a^{-1}\right\rangle_{\mathrm{alg}}
$$

and

$$
a b^{\prime}=q b^{\prime} a .
$$

We have seen in Section 9 that $R=\left\langle Q, Q^{-1}, X\right\rangle_{\text {alg }}$ and the relations among the generators $Q, Q^{-1}, t$ are reduced to

$$
Q Q^{-1}=Q^{-1} Q=1, \quad q X Q-Q X=0, \quad C \text { commutes with } Q, Q^{-1} \text { and } t .
$$

Thus, there exists a $C$-morphism $\varphi: R \rightarrow \mathcal{R}$ of abstract $C$-algebras by (10.17). It follows from (10.15) and difference-differential equations for $Q, X$, the morphism $\varphi$ is qsi morphism. By (10.16), the morphism $\varphi$ is surjective. Since $R$ is simple qsi algebra, the kernel of the qsi morphism $\varphi$ is 0 and the morphism is injective. Therefore the qsi morphism $\varphi$ is an isomorphism.

\section{Generalized Tannaka equivalence of categories}

Let us review classical Picard-Vessiot theory formulated by Tannaka equivalence of two rigid tensor categories. Let $k$ be a differential field of characteristic 0 and we assume the field $C=C_{k}$ of constants of $k$ is algebraically closed. We denote by $\mathcal{D}=k[\partial]$ the ring of linear differential operators. We denote by $(\mathcal{D}$-mod) the category of left $\mathcal{D}$-modules that are finite-dimensional $k$-vector spaces. We know $(\mathcal{D}$-mod) is a rigid tensor category. Namely, for two objects $M_{1}, M_{2} \in \mathrm{ob}\left(\mathcal{D}\right.$-mod), the tensor product $M_{1} \otimes_{k} M_{2}$ and the internal homomorphism $\operatorname{Hom}_{k}\left(M_{1}, M_{2}\right)$ exist in $(\mathcal{D}$-mod). Let $\mathcal{G}$ be a commutative Hopf algebra. Then similarly the category of right $\mathcal{G}$-co-modules that are finite-dimensional as $C$-vector spaces form a rigid tensor category. Let $H$ be an object of the category $(\mathcal{D}$-mod). Let $G$ be the Galois group of Picard-Vessiot ring of the system of linear differential equations determined by $H$. So $G$ is an affine group scheme over $C$. Hence the coordinate ring $C[G]$ is a Hopf algebra.

Theorem 11.1 (van der Put and Singer [19, Theorem 2.33 in 2.4]). The rigid tensor sub-category $\{\{H\}\}$ of the rigid tensor category $(\mathcal{D}$-mod) generated by $H$ is equivalent to the rigid tensor category (comod-C $[G])$ of right $C[G]$-co-modules that are finite-dimensional $C$-vector spaces. 
The result of preceding Sections suggest that we might as well expect a similar result for the Hopf algebra $C\left[\sigma, \sigma^{-1}, \theta^{*}\right]$. To be more precise in the classical differential case, we take the Hopf algebra $C\left[\mathbb{G}_{a}\right]$ that is commutative and co-commutative, and the base field $k$. In the qsi case, the Hopf algebra $C\left[\sigma, \sigma^{-1}, \theta^{*}\right]$ is neither commutative nor co-commutative, and the base field $k$ coincides with $C$. We studied a concrete example of qsi module $M$ in Section 9 . We let $\left(C\left[\sigma, \sigma^{-1}, \theta^{*}\right]\right.$-mod) denote the category of left $C\left[\sigma, \sigma^{-1}, \theta^{*}\right]$-modules of finite $C$-dimension. Since $C\left[\sigma, \sigma^{-1}, \theta^{*}\right]$ is a Hopf algebra, this is naturally a rigid tensor category. Let $\left(M_{1}, \sigma_{1}, \sigma_{1}^{-1}, \theta_{1}^{*}\right)$ and $\left(M_{2}, \sigma_{2}, \sigma_{2}^{-1}, \theta_{2}^{*}\right)$ be two objects of the category. Then $M_{1} \otimes_{C} M_{2}$ is an object on which the Hopf algebra acts through the co-multiplication. Note that we do not have $M_{1} \otimes_{C} M_{2} \simeq M_{2} \otimes_{C} M_{1}$, in general, since the Hopf algebra is not co-commutative. The Hom space $\left(\operatorname{Hom}\left(M_{1}, M_{2}\right), \sigma_{h}, \sigma_{h}^{-1}, \theta_{h}^{*}\right)$ is an internal hom object with respect to

$$
\sigma_{h}(f)=\sigma_{2} \circ f \circ \sigma_{1}^{-1}, \quad \theta_{h}^{(1)}(f)=-\sigma_{2} \circ f \circ \theta_{1}^{(1)}+\theta_{2}^{(1)} \circ f,
$$

where $f \in \operatorname{Hom}\left(M_{1}, M_{2}\right)$. Since $q$ is not a root of unity, $\theta_{h}^{(m)}$ is given by

$$
\theta_{h}^{(m)}(f)= \begin{cases}f & \text { for } m=0, \\ \frac{1}{[m]_{q} !}\left(\theta_{h}^{(1)}\right)^{m}(f) & \text { for } m>0 .\end{cases}
$$

We consider the rigid tensor category $\left(\operatorname{comod}-\mathfrak{H}_{q}\right)$ of right $\mathfrak{H}_{q}$-co-modules that are finite-dimensional $C$-vector spaces. The results of Sections 9 and 10 would imply the following

EXPECTATION 11.2. - We denote by $\{\{M\}\}$ the rigid tensor sub-category of $\left(C\left[\sigma, \sigma^{-1}, \theta^{*}\right]\right.$-mod $)$ generated by the left qsi-module $M$. Then the rigid tensor category $\{\{M\}\}$ is equivalent to the rigid tensor category $\left(\operatorname{comod}-\mathfrak{H}_{q}\right)$ of right $\mathfrak{H}_{q}$-co-modules that are finite-dimensional $C$-vector spaces.

The Expectation is too naive. It is false but it is not so absurd. Since the Hopf algebra $C\left[\sigma, \sigma^{-1}, \theta^{*}\right]$ is neither commutative nor co-commutative, the arguments for the commutative and co-commutative Hopf algebra $C\left[\mathbb{G}_{a}\right]$ require subtle modifications. We prove a corrected version of Expectation 11.2 in Sections 15 and 16 of Part III.

ObSERVATION 11.3. - We have a partial Galois correspondence between the elements of the two sets.

(1) The set of quotient C-Hopf algebras of $\mathfrak{H}_{q}$ :

$$
\mathfrak{H}_{q}, \mathfrak{H}_{q} / I, C
$$

with the sequences of the quotient morphisms inclusions

$$
\mathfrak{H}_{q} \longrightarrow \mathfrak{H}_{q} / I \longrightarrow C,
$$


where $I$ is the bilateral ideal of the Hopf algebra $\mathfrak{H}_{q}$ generated by $v$.

(2) The sub-set of intermediate qsi division rings of $K / C$ :

$$
C, C(X), K
$$

with inclusions

$$
C \subset C(X) \subset K
$$

The intermediate qsi division rings $C(Q)$ is not written as the ring of invariants of a Hopf ideal so that our Galois correspondence is partial.

The extensions $K / C$ and $K / C(X)$ are $q s i$ Picard-Vessiot extensions with respective Galois groups $\mathfrak{H}_{q}$ and $C\left[\mathbb{G}_{a C}\right]$.

\section{Further examples and generalizations}

Looking at the analysis of the qsi module $M=C 1 \oplus C t$, Pierre Cartier [3] discovered that one can generalize the results to every qsi linear equations over $C$. Let us see other examples to understand better what happens. Let us consider two $3 \times 3$ matrices

$$
A=\left[\begin{array}{lll}
q & 1 & 0 \\
0 & q & 0 \\
0 & 0 & 1
\end{array}\right], \quad B=\left[\begin{array}{lll}
0 & 0 & 1 \\
0 & 0 & 0 \\
0 & 0 & 0
\end{array}\right]
$$

so that $A B=q B A$.

As in the in Section 9, we consider

$$
\sigma Y=A Y \quad \text { and } \quad \theta^{(1)} Y=B Y
$$

over $C$, where $Y$ is a $3 \times 3$ unknown matrix. The linear qsi equation is equivalent to considering a 3 -dimensional vector space $V$ equipped with qsimodule structure defined by the $C$-algebra morphism

$$
C\left[\sigma, \sigma^{-1}, \theta^{*}\right] \longrightarrow M_{3}(C)=\operatorname{End}(V), \quad \sigma^{ \pm 1} \longmapsto{ }^{t} A^{ \pm 1}, \theta^{(1)} \longmapsto{ }^{t} B .
$$

The first task is to solve linear qsi equation (12.1) in the qsi algebra $F(\mathbb{Z}, C)[[t]]$. To this end, we set

$$
Y:=\sum_{i=0}^{\infty} X^{i} A_{i} \in M_{3}(F(\mathbb{Z}, C)[[X]])=M_{3}(F(\mathbb{Z}, C))[[X]]
$$

so that $A_{i} \in M_{3}(F(\mathbb{Z}, C))$ for every $i \in \mathbb{N}$. We may also identify

$$
M_{3}(F(\mathbb{Z}, C))=F\left(\mathbb{Z}, M_{3}(C)\right) .
$$


Therefore $A_{i}$ is a function on the set $\mathbb{Z}$ taking values in the set $M_{3}(C)$ of matrices. So

$$
A_{i}=\left[\begin{array}{ccccccc}
\cdots & -2 & -1 & 0 & 1 & 2 & \ldots \\
\ldots & a_{-2}^{(i)} & a_{-1}^{(i)} & a_{0}^{(i)} & a_{1}^{(i)} & a_{2}^{(i)} & \ldots
\end{array}\right]
$$

with $a_{j}^{(i)} \in M_{3}(C)$ for every $i \in \mathbb{N}, j \in \mathbb{Z}$. Substituting (12.2) into (12.1) and comparing coefficients of $t^{i}$, we get recurrence relations among the $A_{i}$ 's

$$
\sigma\left(A_{i}\right)=q^{-i} A A_{i} \quad \theta^{(1)}\left(A_{i+1}\right)=\frac{1}{[i+1]_{q}} B A_{i}
$$

If we solve recurrence relations (12.3) with the initial condition $a_{0}^{(0)}=I_{3}$, since $B^{2}=0, A_{i}=0$ for $i \geqslant 2$ and

$$
\begin{aligned}
& A_{0}=\left[\begin{array}{cccccccc}
\cdots & -2 & -1 & 0 & 1 & 2 & \cdots \\
\cdots & A^{-2} & A^{-1} & I_{3} & A & A^{2} & \ldots
\end{array}\right]=\left[\begin{array}{ccc}
Q & q^{-1} Z Q & 0 \\
0 & Q & 0 \\
0 & 0 & 1
\end{array}\right], \\
& A_{1}=B A_{0}=\left[\begin{array}{lll}
0 & 0 & 1 \\
0 & 0 & 0 \\
0 & 0 & 0
\end{array}\right] .
\end{aligned}
$$

So

$$
Y=A_{0}+X B A_{0}=\left[\begin{array}{ccc}
Q & q^{-1} Z Q & X \\
0 & Q & 0 \\
0 & 0 & 1
\end{array}\right]
$$

where $Z$ is an element of the ring of functions $F(\mathbb{Z}, C)$ taking the value $n$ at $n \in \mathbb{N}$ so that

$$
Z=\left[\begin{array}{lllllll}
\cdots & -2 & -1 & 0 & 1 & 2 & \ldots \\
\cdots & -2 & -1 & 0 & 1 & 2 & \ldots
\end{array}\right]
$$

The solution $Y$ is an invertible element in the matrix $\operatorname{ring} M_{3}(F(\mathbb{Z}, C)[[t]])$. We introduce a qsi $C$-algebra $R$ generated by the entries of the matrices $Y$ and $Y^{-1}$ in the qsi $C$-algebra $F(\mathbb{Z}, C)[[t]]$. To be more concrete we let

$$
R:=C\left\langle Q, Q^{-1}, Z, X\right\rangle_{\text {alg }}
$$

The commutation relations among the generators are

$$
Q Q^{-1}=Q^{-1} Q=1, \quad q X Q=Q X, \quad X(Z+1)=Z X, \quad Z Q-Q Z .
$$

and the operators act as

$$
\begin{aligned}
\sigma(X) & =q X, & \theta^{(1)}(X) & =1, \\
\sigma(Q) & =q Q, & \theta^{(1)}(Q) & =0, \\
\sigma\left(Q^{-1}\right) & =q^{-1} Q^{-1}, & \theta^{(1)}\left(Q^{-1}\right) & =0, \\
\sigma(Z) & =Z+1, & \theta^{(1)}(Z) & =0 .
\end{aligned}
$$


Then the arguments in the previous example show that the $\operatorname{ring} R$ trivializes the qsi module defined by the matrices $A$ and $B$, the qsi ring $R$ is simple and that the ring of constants $C_{R}=C$. The abstract $C$-algebra $R^{\natural}$ has a $C$-algebra morphism $R^{\natural} \rightarrow C$. So we may call it the Picard-Vessiot ring of the qsi module. Of course we can prove the uniqueness.

Now we are going to speak of the Galois group of qsi equation (12.1). The argument of the previous Section, (12.4) and the actions of the operators (12.5), (12.6), (12.7) and (12.8) allow us to prove the following result.

LEMMA 12.1. - The following conditions for a $C$-algebra $T$ and four elements e, $e^{\prime}, f, g \in T$ are equivalent.

(1) There exists a $C$-qsi morphism

$$
\varphi: R \longrightarrow R \otimes_{C} T
$$

such that

$$
\varphi(Q)=e Q, \quad \varphi\left(Q^{-1}\right)=e^{\prime} Q^{-1}, \quad \varphi(Z)=Z+f, \quad \varphi(t)=t+g Q .
$$

(2) The four elements satisfy the following relations.

$$
e e^{\prime}=e^{\prime} e=1, \quad e g=q f g, \quad e f=f e, \quad f g-g f=g .
$$

Lemma 12.1 tells us the universal co-action. To see the co-algebra structure, let $\varphi_{1}: R \rightarrow R \otimes_{C} T$ be the $C$-qsi morphism determined by four elements $e_{1}, e_{1}^{\prime}, f_{1}, g_{1} \in T$ satisfying relations (12.9). We take another $C$-qsi algebra morphism $\varphi_{2}: R \rightarrow R \otimes_{C} T$ defied by four elements $e_{2}, e_{2}^{\prime}, f_{2}, g_{2} \in T$ satisfying relations (12.9). We assume that the subsets $\left\{e_{1}, e_{1}^{\prime}, f_{1}, g_{1}\right\}$ and $\left\{e_{2}, e_{2}^{\prime}, f_{2}, g_{2}\right\}$ of $T$ are element-wisely commutative. Let us compose $\varphi_{1}$ and $\varphi_{2}$.

$$
\begin{aligned}
Q & \mapsto e_{1} Q & \mapsto e_{2}\left(e_{1} Q\right) & =\left(e_{1} e_{2}\right) Q, \\
Q^{-1} & \mapsto e_{1}^{-1} Q^{-1} & \mapsto e_{2}^{-1}\left(e_{1}^{-1} Q^{-1}\right) & =\left(e_{1}^{-1} e_{2}^{-1}\right) Q, \\
Z & \mapsto Z+f_{1} & \mapsto\left(Z+f_{1}\right)+f_{2} & =Z+\left(f_{1}+f_{2}\right), \\
t & \mapsto t+g_{1} Q & \mapsto\left(t+g_{1} Q\right)+g_{2} e_{1} Q & =t+\left(e_{1} g_{2}+g_{1}\right) Q .
\end{aligned}
$$

Let us now set

$$
\mathfrak{H}:=C\left\langle e, e^{\prime}, f, g\right\rangle_{\text {alg }},
$$

where we assume that the elements e, $e^{\prime}, f, g$ satisfy only relations (12.9) so that we have an isomorphism

$$
R^{\natural} \simeq \mathfrak{H}, \quad Q \longmapsto e, Q^{-1} \longmapsto e^{\prime}, Z \longmapsto f, t \longmapsto g
$$

as abstract $C$-algebras. It follows from the result above of the composition of $\varphi_{1}$ and $\varphi_{2}$ that

$$
\Delta: \mathfrak{H} \longrightarrow \mathfrak{H} \otimes_{C} \mathfrak{H}
$$


with

$\Delta(e)=e \otimes e, \quad \Delta\left(e^{\prime}\right)=e^{\prime} \otimes e^{\prime}, \quad \Delta(f)=f \otimes 1+1 \otimes f, \quad \Delta(g)=g \otimes 1+e \otimes g$ defines a $C$-algebra morphism and together with a $C$-algebra morphism

$$
\epsilon: \mathfrak{H} \longrightarrow C, \quad \text { with } \epsilon(e)=\epsilon\left(e^{\prime}\right)=1, \epsilon(f)=\epsilon(g)=0
$$

makes $\mathfrak{H}$ a Hopf algebra over $C$.

The Galois group of the rank 3 qsi module is the Hopf algebra $\mathfrak{H}$.

We add another example.

Example 12.2. - We consider matrices

$$
A=\left[\begin{array}{cc}
l q & 0 \\
0 & l
\end{array}\right], \quad B=\left[\begin{array}{ll}
0 & 1 \\
0 & 0
\end{array}\right] \in M_{2}(C),
$$

where $l$ is an element of the field $C$. Since $A B=q B A$, the $C$-algebra morphism

$$
C\left[\sigma, \sigma^{-1}, \theta^{*}\right] \longrightarrow M_{2}(C), \quad \sigma^{ \pm 1} \longmapsto{ }^{t} A^{ \pm 1}, \theta^{(1)} \longmapsto{ }^{t} B
$$

defines a 2-dimensional qsi module. We assume that $q$ and $l$ are linearly independent over $\mathbb{Q}$.

We do not give details here as it is useless to repeat the arguments.

(1) The solution matrix in $M_{2}(F(\mathbb{Z}, C)[[t]])$ is

$$
\left[\begin{array}{cc}
L Q & t \\
0 & L
\end{array}\right]
$$

where

$$
L=\left[\begin{array}{llllll}
\cdots & -1 & 0 & 1 & 2 & \cdots \\
\cdots & l^{-1} & 1 & l & l^{2} & \cdots
\end{array}\right] \in F(\mathbb{Z}, C) .
$$

(2) The Picard-Vessiot ring is

$$
C\left\langle Q, Q^{-1}, L, L^{-1}, t\right\rangle_{\text {alg }}
$$

with commutation relations

$$
\begin{gathered}
Q Q^{-1}=Q^{-1} Q=1, \quad L L^{-1}=L^{-1} L=1, \quad Q L=L Q, \\
Q X=q X Q, \quad L X=l X L .
\end{gathered}
$$

Actions of operators:

$$
\begin{aligned}
& \sigma Q=q Q \text {, } \\
& \sigma\left(Q^{-1}\right)=q^{-1} Q^{-1}, \\
& \sigma L=l L, \\
& \sigma\left(L^{-1}\right)=l^{-1} L^{-1} \text {, } \\
& \sigma(X)=q X \\
& \theta^{(1)} Q=0, \\
& \theta^{(1)}\left(Q^{-1}\right)=0 \text {, } \\
& \theta^{(1)}(L)=0 \text {, } \\
& \theta^{(1)}\left(L^{-1}\right)=0 \text {, } \\
& \theta^{(1)}(X)=1 \text {. }
\end{aligned}
$$


(3) The Galois group is the Hopf algebra

$$
\mathfrak{H}:=C\left\langle e, e^{-1}, g, h, h^{-1}\right\rangle_{\text {alg }},
$$

satisfying commutation relations

$$
e e^{-1}=e^{-1} e=1, \quad h h^{-1}=h^{-1} h=1, \quad e g=q g e, \quad h g=l g h .
$$

Co-algebra structure $\Delta: \mathfrak{H} \rightarrow \mathfrak{H} \otimes_{C} \mathfrak{H}:$

$$
\Delta\left(e^{ \pm 1}\right)=e^{ \pm 1} \otimes e^{ \pm 1}, \Delta\left(h^{ \pm 1}\right)=h^{ \pm 1} \otimes h^{ \pm 1}, \Delta(g)=g \otimes 1+e \otimes g .
$$

The co-unit $\epsilon: \mathfrak{H} \rightarrow C$ is given by

$$
\epsilon(e)=\epsilon\left(e^{-1}\right)=\epsilon(h)=\epsilon\left(h^{-1}\right)=1, \quad \epsilon(g)=0 .
$$

The last example is inspired of work of Masatoshi Noumi [18] on the quantization of hypergeometric functions. His idea is that $q$-hypergeometric functions should live on the quantized Grassmannians. Namely, he quantizes the framework of Gelfand of defining general hypergeometric functions.

Example 12.3. - Let $V$ be the natural 2-dimensional representation of $U_{q}\left(s l_{2}\right)$ over $C$. Hence $V$ is a left $U_{q}\left(s l_{2}\right)$-module. So we can speak of the Picard-Vessiot extension $R / C$ attached to the left $U_{q}\left(s l_{2}\right)$-module $V$. The argument in the examples so far studied allows us to guess that $R$ is given by

$$
R:=C\langle a, b, c, d\rangle_{\text {alg }} .
$$

with relations

$$
\begin{gathered}
a b=q b a, \quad b d=q d b, \quad a c=q c a, \quad c d=q d c, \quad b c=c b, \\
a d-d a=\left(q+q^{-1}\right) b c, \quad a d-q b c=1 .
\end{gathered}
$$

Imagine a matrix

$$
\left[\begin{array}{ll}
a & b \\
c & d
\end{array}\right]
$$

and on the space of matrices, the quantum group or Hopf algebra $U_{q}\left(s l_{2}\right)$ operates from right.

Let us recall the definitions. The Hopf algebra $U_{q}\left(s l_{2}\right)=C\left\langle q^{ \pm \frac{H}{2}}, X, Y\right\rangle$ is generated by four elements

$$
1, \quad q^{\frac{H}{2}}, \quad q^{-\frac{H}{2}}, \quad X, \quad Y
$$

over $C$ satisfying the commutation relations

$$
\begin{gathered}
q^{\frac{H}{2}} q^{-\frac{H}{2}}=q^{-\frac{H}{2}} q^{\frac{H}{2}}=1, \quad q^{\frac{H}{2}} X q^{-\frac{H}{2}}=q^{2} X \\
q^{\frac{H}{2}} Y q^{-\frac{H}{2}}=q^{-2} Y, \quad[X, Y]=\frac{q^{H}-q^{-H}}{q-q^{-1}} .
\end{gathered}
$$


The co-algebra structure $\Delta: U_{q}\left(s l_{2}\right) \rightarrow U_{q}\left(s l_{2}\right) \otimes_{C} U_{q}\left(s l_{2}\right)$ is given by $\Delta\left(q^{ \pm \frac{H}{2}}\right)=q^{ \pm \frac{H}{2}} \otimes q^{ \pm \frac{H}{2}}, \quad \Delta(X)=X \otimes 1+q^{\frac{H}{2}} \otimes X, \quad \Delta(Y)=Y \otimes q^{-\frac{H}{2}}+1 \otimes Y$. We define the co-unit $\epsilon: U_{q}\left(s l_{2}\right) \rightarrow C$ by

$$
\epsilon\left(q^{ \pm \frac{H}{2}}\right)=1, \quad \epsilon(X)=\epsilon(Y)=0 .
$$

See S. Majid [10, 3.2] for example.

The $C$-algebra $R$ is a $U_{q}\left(s l_{2}\right)$-module algebra by the action of $U_{q}\left(s l_{2}\right)$ on $R$ defined by

$q^{ \pm \frac{H}{2}}\left[\begin{array}{ll}a & b \\ c & d\end{array}\right]=\left[\begin{array}{cc}q^{ \pm \frac{1}{2}} a & q^{\mp \frac{1}{2}} b \\ q^{ \pm \frac{1}{2}} & q^{\mp \frac{1}{2}} d\end{array}\right], \quad X\left[\begin{array}{ll}a & b \\ c & d\end{array}\right]=\left[\begin{array}{ll}0 & a \\ 0 & c\end{array}\right], \quad Y\left[\begin{array}{ll}a & b \\ c & d\end{array}\right]=\left[\begin{array}{ll}b & 0 \\ d & 0\end{array}\right]$.

We had not exactly examined but we believed

(1) The algebra extension $R / C$ is the Picard-Vessiot extension for the $U_{q}\left(s l_{2}\right)$-module $V$.

(2) The Galois group is the Hopf algebra on the abstract $C$-algebra $R$ with adjunction of the co-algebra structure defined by

$$
\begin{aligned}
\Delta(a) & =a \otimes a+b \otimes c, & \Delta(b) & =a \otimes b+b \otimes d, \\
\Delta(c) & =c \otimes a+d \otimes b, & \Delta(d) & =c \otimes b+d \otimes d
\end{aligned}
$$

and the co-unit $\epsilon: R \rightarrow C$ with

$$
\epsilon(a)=\epsilon(d)=1, \quad \epsilon(b)=\epsilon(c)=0 .
$$

Indeed, the exact consequence that follows from a general theory. See Remark 16.3.

\section{Part III. Hopf-algebraic interpretations}

This part is devoted to giving Hopf-algebraic interpretations to some of those results on linear equations with constant coefficients which have been obtained so far. The restriction "constant coefficients" makes the situation quite simple, since differential modules are then quantized simply to modules over a Hopf algebra, say $\mathcal{H}$. It is shown in Section 13 that given a finite-dimensional $\mathcal{H}$-module $M$, the left rigid, abelian tensor category $\{\{M\}\}$ generated by $M$ is isomorphic, by a standard duality, to the category $\left(\operatorname{comod}-\mathcal{H}_{\pi}^{\circ}\right)$ of finite-dimensional co-modules over what we call the co-representation Hopf algebra $\mathcal{H}_{\pi}^{\circ}$, where $\pi$ indicates the matrix representation of $\mathcal{H}$ associated with $M$. Therefore, this Hopf algebra $\mathcal{H}_{\pi}^{\circ}$ plays the role of the Picard-Vessiot quantum group of $M$. In Section 14 we discuss the qualification for Picard-Vessiot rings: which properties should an $\mathcal{H}$-module algebra have, to be called a Picard-Vessiot ring for a given $M$ ? We will see 
that this question (for general $\mathcal{H}$ ) is subtle, and such a ring is not necessarily unique in contrast to the classical, commutative situation. It looks that $\mathcal{H}_{\pi}^{\circ}$ may be called a Picard-Vessiot ring for $M$. We prove that $\mathcal{H}_{\pi}^{\circ}$ has some of the properties which would be required for such a ring, and in addition, that some objects that generalize $\mathcal{H}_{\pi}^{\circ}$, as well, have them; the latter already implies the non-uniqueness above. Indeed, it does happen that an $\mathcal{H}$-module algebra which even has all reasonably required properties is not unique, as will be shown by explicit examples in the last Section 17. Another contrast to the classical theory arises for the tensor-equivalence $\{\{M\}\} \approx\left(\operatorname{comod}-\mathcal{H}_{\pi}^{\circ}\right)$ mentioned above. Since $\mathcal{H}_{\pi}^{\circ}$ is the trivial $\mathcal{H}_{\pi}^{\circ}$-torsor one might expect that the tensor-equivalence can be realized by the torsor as in the classical theory. But this is not the case in general, as will be explained in Section 15; the result modifies correctly Expectation 11.2. The remaining Section 16 reviews some examples from Part II, refining the results using the arguments in the present part.

Throughout in this Part III we work over a fixed, arbitrary field $C$. Vector spaces, (co-)algebras and Hopf algebras are supposed to be those over $C$. Given a vector space $V$, we let $V^{*}$ denote the dual vector space. The unadorned $\otimes$ denotes the tensor product over $C$.

\section{The co-representation Hopf algebra $\mathcal{H}_{\pi}^{\circ}$}

Let $\mathcal{H}$ be a Hopf algebra. Let $\mathcal{H}^{\circ}$ denote the dual Hopf algebra [20, Section 6.2]. Thus, $\mathcal{H}^{\circ}$ is the filtered union of the finite-dimensional coalgebras $(\mathcal{H} / I)^{*}$ in $\mathcal{H}^{*}$,

$$
\mathcal{H}^{\circ}=\bigcup_{I}(\mathcal{H} / I)^{*},
$$

where $I$ runs over the set of all co-finite ideals of $\mathcal{H}$; note that $(\mathcal{H} / I)^{*}$ is the co-algebra dual to the finite-dimensional quotient algebra $\mathcal{H} / I$ of $\mathcal{H}$.

For a representation-theoretic interpretation of $\mathcal{H}^{\circ}$, note that each $I$ coincides with the kernel of some matrix representation, $\pi: \mathcal{H} \rightarrow M_{n}(C)$, of $\mathcal{H}$. Then one will recall that $(\mathcal{H} / I)^{*}$ coincides with the image

$$
\operatorname{cf}(\pi)=\operatorname{Im}\left(\pi^{*}\right)
$$

of the dual $\pi^{*}: M_{n}(C)^{*} \rightarrow \mathcal{H}^{*}$ of $\pi$. Let $\left\{e_{i j}^{*}\right\}$ denote the dual basis of the basis $\left\{e_{i j}\right\}$ of $M_{n}(C)$ consisting of the matrix units $e_{i j}$, and set $c_{i j}=\pi^{*}\left(e_{i j}^{*}\right)$. Then $\operatorname{cf}(\pi)$ is a co-algebra spanned by $c_{i j}$; it may be called the coefficient co-algebra of $\pi$, whose structure is determined by

$$
\Delta\left(c_{i j}\right)=\sum_{k=1}^{n} c_{i k} \otimes c_{k j}, \quad \epsilon\left(c_{i j}\right)=\delta_{i j} .
$$


We present as

$$
\mathbf{Y}_{\pi}=\left[c_{i j}\right]_{1 \leqslant i, j \leqslant n}=\left[\begin{array}{ccc}
c_{11} & c_{12} & \ldots \\
c_{21} & c_{22} & \ldots \\
\vdots & \vdots & \ddots
\end{array}\right],
$$

and call this the co-representation matrix of $\pi$. One sees that $\operatorname{cf}(\pi)=\operatorname{cf}\left(\pi^{\prime}\right)$, if matrix representations $\pi$ and $\pi^{\prime}$ are equivalent. We have

$$
\mathcal{H}^{\circ}=\bigcup_{\pi} \operatorname{cf}(\pi),
$$

where $\pi$ runs over the set of all equivalence classes of matrix representations of $\mathcal{H}$. Given a matrix representation $\pi$, the image $S(\operatorname{cf}(\pi))$ of $\operatorname{cf}(\pi)$ by the antipode $S$ equals the coefficient co-algebra $\mathrm{cf}\left(\pi^{t}\right)$ of the transpose $\pi^{t}$ of $\pi$. Given another matrix representation $\pi^{\prime}$, we have $\operatorname{cf}(\pi) \subset \operatorname{cf}\left(\pi^{\prime}\right)$ if and only if $\pi$ is a sub-quotient of the direct sum $\pi^{\prime} \oplus \cdots \oplus \pi^{\prime}$ of some copies of $\pi^{\prime}$.

Let $(\mathcal{H}$-mod $)\left(\right.$ resp., $\left.\left(\operatorname{comod}-\mathcal{H}^{\circ}\right)\right)$ denote the category of left $\mathcal{H}$-modules (resp., right $\mathcal{H}^{\circ}$-co-modules) of finite dimension. These two categories are $C$-linear abelian tensor categories. They are both left rigid. Indeed, given an object $M$, the dual $M^{*}$ is naturally a right $\mathcal{H}$-module (resp., a left $\mathcal{H}^{\circ}$-comodule). This, with the side switched through the antipode, gives the left dual of $M$. We have a $C$-linear tensor-isomorphism

$$
(\mathcal{H}-\bmod ) \simeq\left(\operatorname{comod}-\mathcal{H}^{\circ}\right),
$$

which is given by the following one-to-one correspondence between the structures defined on a fixed vector space $M$ of finite dimension. Given an $\mathcal{H}^{\circ}$ co-module structure $\rho: M \rightarrow M \otimes \mathcal{H}^{\circ}$, we present it as

$$
\rho(m)=\sum_{(m)} m_{(0)} \otimes m_{(1)},
$$

following the notation of [20, Section 2.0]. Then the corresponding $\mathcal{H}$-module structure is defined by

$$
h m=\sum_{(m)} m_{(0)}\left\langle m_{(1)}, h\right\rangle, \quad m \in M, h \in \mathcal{H} .
$$

Here and in what follows, $\langle\cdot, \cdot\rangle$ denotes the canonical pairing with values in $C$. Conversely, given an $\mathcal{H}$-module structure on $M$, or a matrix representation $\pi: \mathcal{H} \rightarrow M_{n}(C)$ with respect to some basis $\left\{m_{i}\right\}_{1 \leqslant i \leqslant n}$ of $M$, the corresponding $\mathcal{H}^{\circ}$-co-module structure is defined by

$$
\rho\left(m_{j}\right)=\sum_{i=1}^{n} m_{i} \otimes c_{i j}, \quad 1 \leqslant j \leqslant n,
$$


where $\left[c_{i j}\right]_{1 \leqslant i, j \leqslant n}$ is the co-representation matrix of $\pi$. See [20, Section 2.1]. Let $\mathrm{m}$

$$
\mathbf{Y}_{\pi}(h)=\left[\left\langle c_{i j}, h\right\rangle\right]_{1 \leqslant i, j \leqslant n}, \quad h \in \mathcal{H} .
$$

Then we see from (13.1) and (13.5) that for every $h \in H$,

$$
\begin{gathered}
{\left[\begin{array}{llll}
h m_{1} & h m_{2} & \ldots & h m_{n}
\end{array}\right]=\left[\begin{array}{llll}
m_{1} & m_{2} & \ldots & m_{n}
\end{array}\right] \mathbf{Y}_{\pi}(h) ;} \\
h \mathbf{Y}_{\pi}=\mathbf{Y}_{\pi} \mathbf{Y}_{\pi}(h), \\
\text { or }\left[\begin{array}{lllll}
h c_{i 1} & h c_{i 2} & \ldots & h c_{i n}
\end{array}\right]=\left[\begin{array}{llll}
c_{i 1} & c_{i 2} & \ldots & c_{i n}
\end{array}\right] \mathbf{Y}_{\pi}(h), \quad 1 \leqslant i \leqslant n .
\end{gathered}
$$

In view of Definition 10.2, we say that an $n \times n$ matrix $\mathbf{Y}$ with entries in a left $\mathcal{H}$-module algebra $\mathcal{R}$ is a matrix of fundamental system of solutions for $M$ (or for $\pi$ ), if

(1) $h \mathbf{Y}=\mathbf{Y} \mathbf{Y}_{\pi}(h)$ for all $h \in \mathcal{H}$, and

(2) $\mathbf{Y}$ has an inverse $\mathbf{Y}^{-1}$ in $M_{n}(\mathcal{R})$.

For example, the co-representation matrix $\mathbf{Y}_{\pi}$ of $\pi$ is such a matrix. In fact, the preceding argument ensures (1), while Remark 14.2 below ensures (2). The matrix $\mathbf{Y}$ depends on the choice of a $C$-basis of $M$. But by basis change, the matrix changes only by conjugation by some invertible matrix in $M_{n}(C)$.

Let $M \in \mathrm{ob}(\mathcal{H}$-mod $)$. Let $\{\{M\}\}$ denote the left rigid, abelian tensor subcategory of $(\mathcal{H}$-mod) generated by $M$. It is a full sub-category consisting of those objects which are sub-quotients of some direct sum $L_{1} \oplus \cdots \oplus L_{s}, s \geqslant 0$, of tensor products $L_{i}=X_{i 1} \otimes \cdots \otimes X_{i, t_{i}}, t_{i} \geqslant 0$, where $X_{i j}$ is either $M, M^{*}$, $M^{* *}$ or some further iterated left-dual $M^{* * \cdots *}$ of $M$.

Choose a matrix representation $\pi$ associated with $M$, and define $\mathcal{H}_{\pi}^{\circ}$ to be the smallest Hopf sub-algebra of $\mathcal{H}^{\circ}$ that includes $\operatorname{cf}(\pi)$. If $\left[c_{i j}\right]_{1 \leqslant i, j \leqslant n}$ is the co-representation matrix of $\pi$, then $\mathcal{H}_{\pi}^{\circ}$ is the sub-algebra of $\mathcal{H}^{\circ}$ which is generated by the images

$$
S^{k}\left(c_{i j}\right), \quad k \geqslant 0, \quad 1 \leqslant i, j \leqslant n
$$

of all $c_{i j}$ by iterated antipode. We call $\mathcal{H}_{\pi}^{\circ}$ the co-representation Hopf algebra of $\pi$, or of $M$. The category (comod- $\mathcal{H}_{\pi}^{\circ}$ ) of finite-dimensional right $\mathcal{H}_{\pi}^{\circ}$-comodules is regarded as a full sub-category of $\left(\operatorname{comod}-\mathcal{H}^{\circ}\right)$.

We now reach the following standard result, which is easy to see.

Proposition 13.1. — The isomorphism (13.3) restricts to the $C$-linear tensor-isomorphism

$$
\{\{M\}\} \simeq\left(\operatorname{comod}-\mathcal{H}_{\pi}^{\circ}\right)
$$




\section{Qualification for non-commutative Picard-Vessiot rings}

Let $M \in \mathrm{ob}(\mathcal{H}$-mod), and keep the notation as above. In view of Proposition 13.1 we see

(I) $\mathcal{H}_{\pi}^{\circ}$ plays the role of the Picard-Vessiot quantum group of $M$.

Note that the isomorphism (13.3) extends to a $C$-linear tensor-isomorphism between the category of locally finite left $\mathcal{H}$-modules and the category (comod- $\mathcal{H}^{\circ}$ ) of right $\mathcal{H}^{\circ}$-co-modules of possibly infinite dimension. Since $\mathcal{H}_{\pi}^{\circ}$ is naturally in $\left(\operatorname{comod}-\mathcal{H}^{\circ}\right)$, it is a left $\mathcal{H}$-module, and is indeed a left $\mathcal{H}$-module algebra. The $\mathcal{H}$-module structure is explicitly given by

$$
h x=\sum_{(x)} x_{(1)}\left\langle x_{(2)}, h\right\rangle, \quad h \in \mathcal{H}, x \in \mathcal{H}_{\pi}^{\circ} .
$$

We say that $M$ is trivialized by a left $\mathcal{H}$-module algebra $\mathcal{R}$ (or $\mathcal{R}$ trivializes $M)$, if the $\mathcal{H}$-module $M \otimes \mathcal{R}$ of tensor product is isomorphic to the direct sum of some copies of $\mathcal{R}$. The same term was used in Observation 9.5, replacing the $M \otimes \mathcal{R}$ here with $\mathcal{R} \otimes M$ with conversely ordered tensor factors; it will be shown in Remark 14.5(3) that the order does not matter under some assumption that is satisfied by our examples.

Proposition 14.1. - We have the following.

(II) The $\mathcal{H}$-module $M$ is trivialized by $\mathcal{H}_{\pi}^{\circ}$.

(III) The $\mathcal{H}$-module algebra $\mathcal{H}_{\pi}^{\circ}$ is simple in the sense that it includes no non-trivial $\mathcal{H}$-stable ideals. Moreover, it includes no non-trivial $\mathcal{H}$-stable right ideals.

Proof. -

(II). - Let $(M) \otimes \mathcal{H}_{\pi}^{\circ}\left(=\left(\mathcal{H}_{\pi}^{\circ}\right)^{\operatorname{dim} M}\right)$ denote the left $\mathcal{H}$-module for which $\mathcal{H}$ acts on the single factor $\mathcal{H}_{\pi}^{\circ}$. Then a desired isomorphism

$$
M \otimes \mathcal{H}_{\pi}^{\circ} \stackrel{\simeq}{\longrightarrow}(M) \otimes \mathcal{H}_{\pi}^{\circ}
$$

is given, with the notation (13.4), by $m \otimes x \mapsto \sum_{(m)} m_{(0)} \otimes m_{(1)} x$; the inverse is given by $m \otimes x \mapsto \sum_{(m)} m_{(0)} \otimes S\left(m_{(1)}\right) x$.

(III). - In general, every Hopf sub-algebra $\mathfrak{H}$ of $\mathcal{H}^{\circ}$ is regarded naturally as a left $\mathcal{H}$-module algebra, and is simple. Indeed, $\mathfrak{H}$ is an $\mathfrak{H}$-Hopf module [20, Section 4.1], whence by [20, Theorem 4.0.5], it includes no non-trivial $\mathcal{H}$ stable right ideals. 
Remark 14.2. - In view of Properties (II) and (III) above one would call $\mathcal{H}_{\pi}^{\circ}$ a Picard-Vessiot ring for $M$. But let us see that the algebra is generated by the entries of all

$$
\mathbf{Y}_{0}=\mathbf{Y}_{\pi}=\left[c_{i j}\right], \quad \mathbf{Y}_{1}=\left[S\left(c_{i j}\right)\right], \quad \mathbf{Y}_{2}=\left[S^{2}\left(c_{i j}\right)\right], \quad \ldots
$$

Note that if $k$ is even, $\mathbf{Y}_{k}$ and $\mathbf{Y}_{k+1}$ are inverse to each other, while if $k$ is odd, the transposes $\mathbf{Y}_{k}^{t}$ and $\mathbf{Y}_{k+1}^{t}$ are inverse to each other. Thus the circumstance is naturally different from the commutative situation in which the Picard-Vessiot ring is generated by the entries of $\mathbf{Y}$ and $\mathbf{Y}^{-1}$, where $\mathbf{Y}$ is a matrix of fundamental system of solutions. Indeed, in our situation as well, $\mathbf{Y}_{\pi}$ is a matrix of fundamental system of solutions, and the subalgebra $\mathcal{R}$ of $\mathcal{H}_{\pi}^{\circ}$ generated by the entries of $\mathbf{Y}_{0}=\mathbf{Y}_{\pi}$ and $\mathbf{Y}_{1}=\mathbf{Y}_{\pi}^{-1}$ is a left $\mathcal{H}$ module algebra which trivializes $M$. But it may loose the property probably required for Picard-Vessiot rings that it should be an $\mathcal{H}_{\pi}^{\circ}$-torsor; see the second paragraph following Proposition 14.3 below. Even if $\mathcal{H}_{\pi}^{\circ}$ happens to coincide with the $\mathcal{R}$ above, it may not be the unique "Picard-Vessiot ring" for $M$, as will be shown by explicit examples in Section 17 . We are now going to prove that Properties (II) and (III) are possessed by some $\mathcal{H}$-module algebras that generalize the Hopf algebra $\mathcal{H}_{\pi}^{\circ}$; those generalized objects are used as well to present the examples referred to above.

Proposition 14.1 is generalized by the following.

Proposition 14.3. - Let $\mathfrak{H}$ be an arbitrary Hopf sub-algebra of $\mathcal{H}^{\circ}$ including $\mathcal{H}_{\pi}^{\circ}$. Let $\mathcal{R}$ be a non-zero right $\mathfrak{H}$-co-module algebra, which is naturally regarded as a left $\mathcal{H}$-module algebra.

(II') If $\mathcal{R}$ is cleft, then the $\mathcal{H}$-module $M$ is trivialized by $\mathcal{R}$.

$\left(\mathrm{III}^{\prime}\right)$ If $\mathcal{R}$ is an $\mathfrak{H}$-torsor, then it includes no non-trivial $\mathcal{H}$-stable right ideals.

To prove the Proposition in a generalized situation, suppose that $\mathfrak{H}$ is an arbitrary Hopf algebra, and let $\mathcal{R}$ be a non-zero right $\mathfrak{H}$-co-module algebra.

First, as for $\left(\mathrm{III}^{\prime}\right)$, we say that $\mathcal{R}$ is an $\mathfrak{H}$-torsor (or an $\mathcal{H}$-Galois extension [15, Definition 8.1.1] over $C$ ), if the map $\mathcal{R} \otimes \mathcal{R} \rightarrow \mathcal{R} \otimes \mathfrak{H}$ given by $x \otimes y \mapsto \sum_{(y)} x y_{(0)} \otimes y_{(1)}$, with the same notation as in (13.4), is bijective. Note that $\mathfrak{H}$ itself is naturally an $\mathfrak{H}$-torsor, whence (III') generalizes (III) of Proposition 14.1. This (III') follows since we see from [15, Theorem 8.5.6] that every $\mathfrak{H}$-torsor includes no non-trivial $\mathcal{H}$-stable right ideals.

Next, as for $\left(\mathrm{II}^{\prime}\right)$, recall from [15, Definition 7.2.1] that $\mathcal{R}$ is said to be cleft, if there exists an $\mathfrak{H}$-co-module map $\phi: \mathfrak{H} \rightarrow \mathcal{R}$ that is invertible with respect to the convolution-product [15, Definition 1.2.1]; the inverse $\phi^{-1}$ is 
then characterized by

$$
\sum_{(x)} \phi\left(x_{(1)}\right) \phi^{-1}\left(x_{(2)}\right)=\epsilon(x) 1=\sum_{(x)} \phi^{-1}\left(x_{(1)}\right) \phi\left(x_{(2)}\right), \quad x \in \mathfrak{H} .
$$

Note that $\mathfrak{H}$ itself is cleft with respect to $\phi=\mathrm{Id}$, the identity, and $\phi^{-1}=S$, the antipode. Hence $\left(\mathrm{II}^{\prime}\right)$ generalizes (II) of Proposition 14.1; note that $M$ is regarded naturally as a right $\mathfrak{H}$-co-module since $\mathfrak{H} \supset \mathcal{H}_{\pi}^{\circ}$ by assumption. We see that $\left(\mathrm{II}^{\prime}\right)$ follows from the next Lemma.

LEMmA 14.4. - Suppose that $\mathcal{R}$ is cleft. Given a right $\mathfrak{H}$-co-module $N$, an $\mathfrak{H}$-co-module isomorphism

$$
N \otimes \mathcal{R} \stackrel{\simeq}{\longrightarrow}(N) \otimes \mathcal{R}
$$

(analogous to (14.2)) is given by $n \otimes x \mapsto \sum_{(n)} n_{(0)} \otimes \phi\left(n_{(1)}\right) x$, where $N \otimes \mathcal{R}$ denotes the $\mathfrak{H}$-co-module of tensor product, and $(N) \otimes \mathcal{R}$ denotes the $\mathfrak{H}$-comodule for which $\mathfrak{H}$ co-acts on the single factor $\mathcal{R}$.

Proof. - The inverse is given by $n \otimes x \mapsto \sum_{(n)} n_{(0)} \otimes \phi^{-1}\left(n_{(1)}\right) x$.

It is known (see [15, Theorem 8.2.4]) that the cleft $\mathfrak{H}$-torsors coincide with each of the following two classes of non-zero right $\mathfrak{H}$-co-module algebras:

- Those $\mathfrak{H}$-torsors which are isomorphic to $\mathfrak{H}$ as $\mathfrak{H}$-co-module;

- The cleft right $\mathfrak{H}$-co-module algebras $\mathcal{R}=(\mathcal{R}, \rho)$ such that $\mathcal{R}^{\text {cor }}=$ $C$, where $\mathcal{R}^{c o \mathfrak{H}}=\{x \in \mathcal{R} \mid \rho(x)=x \otimes 1\}$ denotes the sub-algebra of $\mathcal{R}$ consisting of all $\mathfrak{H}$-co-invariants.

We remark that given an $\mathfrak{H}$-torsor $\mathcal{R}$, an $\mathfrak{H}$-co-module map $\mathfrak{H} \rightarrow \mathcal{R}$ is an isomorphism if and only if it is invertible with respect to the convolutionproduct; see the proof of [15, Theorem 8.2.4].

One sees easily that if $\mathfrak{H}$ is finite-dimensional, then every $\mathfrak{H}$-torsor is necessarily cleft.

Remark 14.5. -

(1). - Let $\mathfrak{H}$ be a Hopf algebra in general. Assume that the antipode $S$ of $\mathfrak{H}$ is bijective. Then the inverse $\bar{S}$ of $S$ is the unique linear endomorphism of $\mathfrak{H}$ such that

$$
\sum_{(x)} x_{(2)} \bar{S}\left(x_{(1)}\right)=\epsilon(x) 1=\sum_{(x)} \bar{S}\left(x_{(2)}\right) x_{(1)}, \quad x \in \mathfrak{H} .
$$

The assumption is satisfied if $\mathfrak{H}$ is finite-dimensional, commutative, co-commutative or pointed [20, Section 8.0].

(2). - Given an algebra $\mathcal{R}$, let $\mathcal{R}^{\text {op }}$ denote the opposite algebra. Then $\mathfrak{H}^{\text {op }}$, given the original co-algebra structure on $\mathfrak{H}$, is a bi-algebra. This $\mathfrak{H}^{\text {op }}$ 
is a Hopf algebra if and only if $S$ is bijective. Suppose that this is the case. Then $\bar{S}$ is the antipode of $\mathfrak{H}^{\text {op }}$. Through $\mathcal{R} \leftrightarrow \mathcal{R}^{\text {op }}$, the (cleft) $\mathfrak{H}$-torsors and the (cleft) $\mathfrak{H}^{\text {op }}$-torsors are in one-to-one correspondence. Let $\mathcal{R}$ be a cleft $\mathfrak{H}$-torsor, and suppose that $\phi: \mathfrak{H} \rightarrow \mathcal{R}$ is an $\mathfrak{H}$-co-module map which is invertible with respect to the convolution-product. Regarded as an $\mathfrak{H}^{\text {op }}$-comodule map $\mathfrak{H}^{\text {op }} \rightarrow \mathcal{R}^{\text {op }}, \phi$ remains invertible (see the second last paragraph preceding this Remark 14.5), whence there uniquely exists a linear map $\bar{\phi}: \mathfrak{H} \rightarrow \mathcal{R}$ such that

$$
\sum_{(x)} \phi\left(x_{(2)}\right) \bar{\phi}\left(x_{(1)}\right)=\epsilon(x) 1=\sum_{(x)} \bar{\phi}\left(x_{(2)}\right) \phi\left(x_{(1)}\right), \quad x \in \mathfrak{H} .
$$

Note that if $\mathcal{R}=\mathfrak{H}$ and $\phi=\mathrm{Id}$, then $\bar{\phi}$ coincides with the $\bar{S}$ above.

(3). - Suppose that we are in the situation of Proposition 14.3 and Lemma 14.4. Assume that the antipode of $\mathfrak{H}$ is bijective, and let $\mathcal{R}$ be a cleft $\mathfrak{H}$-torsor with $\phi, \bar{\phi}$ as above. Given a right $\mathfrak{H}$-co-module $N$, we have an $\mathfrak{H}$-co-module isomorphism analogous to (14.3),

$$
\mathcal{R} \otimes N \stackrel{\simeq}{\longrightarrow}(N) \otimes \mathcal{R}, \quad x \otimes n \longmapsto \sum_{(n)} n_{(0)} \otimes x \phi\left(n_{(1)}\right),
$$

whose inverse is given by $n \otimes x \longmapsto \sum_{(n)} x \bar{\phi}\left(n_{(1)}\right) \otimes n_{(0)}$. In particular, in the situation of Proposition 14.1, if the antipode of $\mathcal{H}_{\pi}^{\circ}$ is bijective, then we have an $\mathcal{H}$-module isomorphism analogous to (14.2),

$$
\mathcal{H}_{\pi}^{\circ} \otimes M \stackrel{\simeq}{\longrightarrow}(M) \otimes \mathcal{H}_{\pi}^{\circ}, \quad x \otimes m \longmapsto \sum_{(m)} m_{(0)} \otimes x m_{(1)} .
$$

In the examples which we will treat in Sections 16 and 17, the Hopf algebras $\mathcal{H}_{\pi}^{\circ}$ are pointed, so that we have the last isomorphism for those.

\section{Tensor-equivalence expected from the classical one}

Let $M \in \mathrm{ob}(\mathcal{H}$-mod $)$, and keep the notation as above. Let us return to Property (I). Recall that in the classical theory, Picard-Vessiot rings give the tensor-equivalences as found in [1, Theorem 8.11], for example; for this, essential is that those rings are torsors. From (I) one may expect an analogous tensor-equivalence $\{\{M\}\} \approx\left(\operatorname{comod}-\mathcal{H}_{\pi}^{\circ}\right)$, since $\mathcal{H}_{\pi}^{\circ}$ is indeed a (trivial) $\mathcal{H}_{\pi}^{\circ}$ torsor. We will see that under some mild assumption, there exists such a tensor-equivalence, for which, however, $\mathcal{H}_{\pi}^{\circ}$ does not act as a torsor any more. The result gives the "corrected version of Expectation 11.2" as referred to just after the Expectation. 
Let us write $\mathcal{R}$ for the trivial $\mathcal{H}_{\pi}^{\circ}$-torsor $\mathcal{H}_{\pi}^{\circ}$, to make its role clearer. Recall that $\mathcal{R}$ has the natural left $\mathcal{H}$-module structure as given by (14.1). Define a right $\mathcal{H}_{\pi}^{\circ}$-co-module structure $\varrho: \mathcal{R} \rightarrow \mathcal{R} \otimes \mathcal{H}_{\pi}^{\circ}$ on $\mathcal{R}$ by

$$
\varrho(x)=\sum_{(x)} x_{(2)} \otimes S\left(x_{(1)}\right), \quad x \in \mathcal{R},
$$

where $S$ denotes the antipode of $\mathcal{H}_{\pi}^{\circ}$. Note that $(\mathcal{R}, \varrho)$ is not an $\mathcal{H}_{\pi}^{\circ}$-torsor nor even a right $\mathcal{H}_{\pi}^{\circ}$-co-module algebra in general. Given $N \in \mathrm{ob}(\mathcal{H}$-mod), endow the left $\mathcal{H}$-module $\mathcal{R} \otimes N$ with the right $\mathcal{H}_{\pi}^{\circ}$-co-module structure induced by $\varrho$. Since the $\mathcal{H}$-module structure on $\mathcal{R}$ commutes with $\varrho$, the $\mathcal{H}$-invariants $(\mathcal{R} \otimes N)^{\mathcal{H}}$ in $\mathcal{R} \otimes N$, consisting of those elements on which $\mathcal{H}$-acts trivially through the co-unit, form an $\mathcal{H}_{\pi}^{\circ}$-sub-co-module.

Lemma 15.1. - Assume that the antipode of $\mathcal{H}_{\pi}^{\circ}$ is bijective. Then $N \mapsto$ $(\mathcal{R} \otimes N)^{\mathcal{H}}$ gives a tensor-equivalence $\{\{M\}\} \approx\left(\operatorname{comod}-\mathcal{H}_{\pi}^{\circ}\right)$.

Proof. - Let $\bar{S}$ denote the inverse of the antipode $S$ of $\mathcal{H}_{\pi}^{\circ}$. Let $N \in$ $\{\{M\}\}$, and regard it as an object also in (comod- $\mathcal{H}_{\pi}^{\circ}$ ) through (13.3). Using the notation (13.4), we see that

$$
\alpha_{N}: N \longrightarrow(\mathcal{R} \otimes N)^{\mathcal{H}}, \quad \alpha_{N}(n)=\sum_{(n)} \bar{S}\left(n_{(1)}\right) \otimes n_{(0)} .
$$

is an isomorphism in $\left(\operatorname{comod}-\mathcal{H}_{\pi}^{\circ}\right)$. Indeed, the last sum is $\mathcal{H}$-invariant since it is seen to be $\mathcal{H}_{\pi}^{\circ}$-co-invariant. The inverse $\alpha_{N}^{-1}$ associates $\sum_{i} \epsilon\left(x_{i}\right) n_{i}$ to an element $\sum_{i} x_{i} \otimes n_{i} \in(\mathcal{R} \otimes N)^{\mathcal{H}}$. The isomorphism $\alpha_{N}$ is natural in $N$. It translates the tensor-isomorphism (13.3) into the tensor-equivalence $F: N \mapsto(\mathcal{R} \otimes N)^{\mathcal{H}}$, whose tensor structure is given by $\alpha_{C}$ and

$$
\begin{aligned}
F(N) \otimes F\left(N^{\prime}\right) & \longrightarrow F\left(N \otimes N^{\prime}\right), \\
\left(\sum_{i} x_{i} \otimes n_{i}\right) \otimes\left(\sum_{j} y_{j} \otimes n_{j}^{\prime}\right) & \longmapsto \sum_{i, j} y_{j} x_{i} \otimes\left(n_{i} \otimes n_{j}^{\prime}\right)
\end{aligned}
$$

for $N, N^{\prime} \in\{\{M\}\}$. Note that the last map, composed with $\alpha_{N} \otimes \alpha_{N}^{\prime}$, coincides with $\alpha_{N \otimes N^{\prime}}$, and is, therefore, an isomorphism.

Assume that $\mathcal{H}$ is co-commutative, and so that $\mathcal{H}_{\pi}^{\circ}=\mathcal{R}$ is commutative. Their antipodes are then involutions. Therefore, $\mathcal{R}$ is a Picard-Vessiot ring, being generated by the entries of $\mathbf{Y}_{\pi}^{ \pm 1}$. Through the antipode of $\mathcal{H}_{\pi}^{\circ}$ transfer the structures of $\mathcal{R}$ onto its copy, which we denote by $\mathcal{R}^{\prime}$. Then $\mathcal{R}^{\prime}$ has the original, natural right $\mathcal{H}_{\pi}^{\circ}$-co-module structure, under which it is the trivial $\mathcal{H}_{\pi}^{\circ}$-torsor. The left $\mathcal{H}$-module structure on $\mathcal{R}^{\prime}$ is given by

$$
h x=\sum_{(x)}\left\langle x_{(1)}, S(h)\right\rangle x_{(2)}, \quad h \in \mathcal{H}, x \in \mathcal{R}^{\prime},
$$


where $S$ denotes the antipode of $\mathcal{H}$. The co-commutativity assumption ensures that $\mathcal{R}^{\prime}$ is a left $\mathcal{H}$-module algebra, and it remains a Picard-Vessiot ring for $M$. The tensor-equivalence above now reads $N \mapsto\left(\mathcal{R}^{\prime} \otimes N\right)^{\mathcal{H}}$, which is, given by the torsor $\mathcal{R}^{\prime}$, of the same form as the classical ones as found in $[1$, Theorem 8.11].

\section{Reviewing some examples}

To review two examples from Part II, let $\mathcal{H}=\mathcal{H}_{q}$ be the Hopf algebra defined by Definition 3.9. Assume that the element $0 \neq q \in C$ is not a root of 1 , unless otherwise stated. We write $t$ for the element $t_{1}$ given in the Definition. Then

$$
\left(\mathcal{H}_{q}=\right) \mathcal{H}=C\left[s, s^{-1}\right] \otimes C[t] .
$$

This means that $\mathcal{H}$ includes the Laurent polynomial algebra $C\left[s, s^{-1}\right]$ and the polynomial algebra $C[t]$ so that the product map $C\left[s, s^{-1}\right] \otimes C[t] \rightarrow \mathcal{H}$ is bijective. The algebra structure on $\mathcal{H}$ is determined by the relation $t s=q s t$. Obviously, $\mathcal{H}$ has

$$
v_{m, n}=s^{m} \frac{t^{n}}{[n]_{q} !}, \quad m \in \mathbb{Z}, n \in \mathbb{N}
$$

as a basis. The co-algebra structure is given by

$$
\Delta\left(v_{m, n}\right)=\sum_{i+j=n} v_{m+j, i} \otimes v_{m, j}, \quad \epsilon\left(v_{m, n}\right)=\delta_{n, 0} .
$$

In particular, $s$ and $s^{-1}$ are grouplikes, and $t$ is $(s, 1)$-primitive, or $\Delta(t)=$ $s \otimes t+t \otimes 1$. Generated by those elements, $\mathcal{H}$ is a pointed Hopf algebra, whose antipode is necessarily bijective. We remark that $s$ and $t$ are denoted in Section 9 by $\sigma$ and $\theta^{(1)}$, respectively.

Define elements $e, f, g \in \mathcal{H}^{*}$ by

$$
\left\langle e, v_{m, n}\right\rangle=q^{m} \delta_{n, 0}, \quad\left\langle f, v_{m, n}\right\rangle=m \delta_{n, 0}, \quad\left\langle g, v_{m, n}\right\rangle=\delta_{n, 1} .
$$

Then we see that

$$
\mathfrak{H}=C\left[e, e^{-1}, f\right] \otimes C[g]
$$

is included in $\mathcal{H}^{\circ}$; this means, just as before, that the two commutative algebras are included in $\mathcal{H}^{\circ}$ so that the product map $C\left[e, e^{-1}, f\right] \otimes C[g] \rightarrow \mathcal{H}^{\circ}$ is injective. By using (16.1) we obtain

$$
e g=q g e, \quad f g-g f=g .
$$


These relations show that $\mathfrak{H}$ is a sub-algebra of $\mathcal{H}^{\circ}$, determining its algebra structure. Since $e$ (resp., $f$ ) is in fact a $C$-valued algebra map (resp., derivation) defined on the quotient Hopf algebra $\mathcal{H} /(t)=C\left[s, s^{-1}\right]$ of $\mathcal{H}$, it follows that

$$
\Delta(e)=e \otimes e, \quad \epsilon(e)=1, \quad \Delta(f)=1 \otimes f+f \otimes 1, \quad \epsilon(f)=0 .
$$

(As an additional remark, $g^{n}, n \geqslant 0$, can be grasped as follows: the quotient co-algebra $\mathcal{Q}$ of $\mathcal{H}$ divided by the right ideal generated by $s-1$ is spanned by the $\infty$-divided power sequence $1, t, t^{2} /[2]_{q} !, \ldots$ The $g^{n}$ are the elements of $\mathcal{Q}^{*}$ given by $\left\langle g^{n}, t^{\ell} /[\ell]_{q} !\right\rangle=\delta_{n, \ell}$.) Since one computes

$$
\left\langle g, v_{m, n} v_{k, \ell}\right\rangle= \begin{cases}1 & \text { if } n=0, \ell=1, \\ q^{k} & \text { if } n=1, \ell=0, \\ 0 & \text { otherwise }\end{cases}
$$

it follows that

$$
\Delta(g)=1 \otimes g+g \otimes e, \quad \epsilon(g)=0 .
$$

By (16.3) and (16.4), $\mathfrak{H}$ is a sub-bi-algebra of $\mathcal{H}^{\circ}$. Indeed, it is a Hopf subalgebra, having the antipode determined by

$$
S(e)=e^{-1}, \quad S(f)=-f, \quad S(g)=-g e^{-1} .
$$

From this explicit form of the antipode we will see that for the following two examples of matrix representations $\pi$ of $\mathcal{H}$, the co-representation Hopf algebra $\mathcal{H}_{\pi}^{\circ}$ is generated by the entries of the co-representation matrix $\mathbf{Y}_{\pi}$ and $e^{-1}=S(e)$; indeed, since $e$ is an entry of $\mathbf{Y}_{\pi}, \mathcal{H}_{\pi}^{\circ}$ is generated by the entries of $\mathbf{Y}_{\pi}^{ \pm 1}$. Note also that the Hopf algebra $\mathfrak{H}$ and those $\mathcal{H}_{\pi}^{\circ}$ which will be obtained as its Hopf sub-algebras are all pointed.

Example 16.1. - Recall from Section 9 the matrix representation $\pi$ of $\mathcal{H}$ determined by

$$
\pi(s)=\left[\begin{array}{ll}
q & 0 \\
0 & 1
\end{array}\right], \quad \pi(t)=\left[\begin{array}{ll}
0 & 0 \\
1 & 0
\end{array}\right] .
$$

These matrices are the transposes of those in Section 9, since the basis of the corresponding $\mathcal{H}$-module is supposed here to be in a row vector, whereas in Section 9 it is supposed to be in a column vector. Since one computes

$$
\begin{aligned}
\pi\left(v_{m, n}\right)= & \begin{cases}{\left[\begin{array}{cc}
q^{m} & 0 \\
0 & 1
\end{array}\right]} & n=0, \\
{\left[\begin{array}{ll}
0 & 0 \\
1 & 0
\end{array}\right]} & n=1, \\
O & n \geqslant 2,\end{cases} \\
& -1426-
\end{aligned}
$$


it follows that

$$
\mathbf{Y}_{\pi}=\left[\begin{array}{ll}
e & 0 \\
g & 1
\end{array}\right]
$$

We conclude that $\mathcal{H}_{\pi}^{\circ}=C\left\langle e^{ \pm 1}, g\right\rangle$, the Hopf sub-algebra of $\mathfrak{H}$ generated by $e^{ \pm 1}$ and $g$.

Direct computations show that the natural left $\mathcal{H}$-module structure on this $C\left\langle e^{ \pm 1}, g\right\rangle$ is given by

$$
s\left[\begin{array}{ll}
e^{ \pm 1} & g
\end{array}\right]=\left[\begin{array}{ll}
q^{ \pm 1} e^{ \pm 1} & q g
\end{array}\right], \quad t\left[\begin{array}{ll}
e^{ \pm 1} & g
\end{array}\right]=\left[\begin{array}{ll}
0 & 1
\end{array}\right] .
$$

One then sees that $e^{ \pm 1} \mapsto Q^{ \pm 1}, g \mapsto t$ give an isomorphism from $C\left\langle e^{ \pm 1}, g\right\rangle$ to the $\mathcal{H}$-module algebra $R=C\left\langle t, Q, Q^{-1}\right\rangle$ alg given in Observation 9.2. As was shown by Proposition 9.13, the Hopf algebra $\mathfrak{H}_{q}$ constructed in Lemma 4.12 co-acts on this last $R$ from the right, so that $R$ is an $\mathfrak{H}_{q^{-}}$ torsor, and the $\mathfrak{H}_{q}$-co-action commutes with the $\mathcal{H}$-action. Note that the Hopf algebra $\mathcal{H}_{\pi}^{\circ}=C\left\langle e^{ \pm 1}, g\right\rangle$ here obtained differs from the $\mathfrak{H}_{q}$ only in that their co-multiplications are opposite to each other. The reason of this difference has been seen in the preceding section: in the quantized situation, the Picard-Vessiot quantum group $\mathcal{H}_{\pi}^{\circ}$ does not act as a torsor which was expected to give the tensor equivalence (13.6). In other words, $\mathfrak{H}_{q}$ is not the Picard-Vessiot quantum group, so that we do not have $\{\{M\}\} \approx\left(\operatorname{comod}-\mathfrak{H}_{q}\right)$ analogous to (13.6). Indeed, Proposition 9.13 does not assert such a tensor equivalence.

Theorem 10.7 has proved that $\mathcal{H}_{\pi}^{\circ}$ is the unique (up to isomorphism) Picard-Vessiot ring for $\pi$. The definition of Picard-Vessiot rings adopted by the theorem will be reproduced in the following section, in which the proved uniqueness will be seen not to hold under some milder (seemingly reasonable) restriction.

Example 16.2. - Recall from Example 12.1 the matrix representation $\pi$ of $\mathcal{H}$ determined by

$$
\pi(s)=\left[\begin{array}{lll}
q & 0 & 0 \\
1 & q & 0 \\
0 & 0 & 1
\end{array}\right], \quad \pi(t)=\left[\begin{array}{lll}
0 & 0 & 0 \\
0 & 0 & 0 \\
1 & 0 & 0
\end{array}\right]
$$


Since one computes

$$
\pi\left(v_{m, n}\right)=\left\{\begin{array}{lccc}
{\left[\begin{array}{ccc}
q^{m} & 0 & 0 \\
m q^{m-1} & q^{m} & 0 \\
0 & 0 & 1
\end{array}\right]} & n=0, \\
{\left[\begin{array}{lll}
0 & 0 & 0 \\
0 & 0 & 0 \\
1 & 0 & 0
\end{array}\right]} & n=1, \\
O & & n \geqslant 2,
\end{array}\right.
$$

it follows that

$$
\mathbf{Y}_{\pi}=\left[\begin{array}{ccc}
e & 0 & 0 \\
\frac{1}{q} e f & e & 0 \\
g & 0 & 1
\end{array}\right] .
$$

We conclude that $\mathcal{H}_{\pi}^{\circ}=\mathfrak{H}$, the Hopf algebra given above by (16.2).

One sees that this $\mathfrak{H}$ is isomorphic, as an $\mathcal{H}$-module algebra, to the $R=$ $C\left\langle Q, Q^{-1}, Z, t\right\rangle_{\text {alg }}$ obtained in Example 12.1 via $e^{ \pm 1} \mapsto Q^{ \pm 1}, f \mapsto Z, g \mapsto t$. As Hopf algebras, $\mathfrak{H}$ and the $A=C\left\langle e, e^{\prime}, f, g\right\rangle_{\text {alg }}$ resulting from Lemma 12.1 coincide except in that their co-multiplications are opposite to each other, again; see the second last paragraph of the preceding example.

Remark 16.3. - Suppose that characteristic $\operatorname{char} C$ of $C$ is not 2, and that $0 \neq q \in C$ is not a root of 1 . Let $\mathcal{H}=U_{q}\left(s l_{2}\right)$ be the quantized enveloping algebra of $s l_{2}$ as given in Example 12.3. This example is essentially the attempt to determine the Hopf algebra $\mathcal{H}_{\pi}^{\circ}$ for the fundamental representation $\pi$ of $\mathcal{H}$. It is known that $\mathcal{H}_{\pi}^{\circ}$ is the coordinate Hopf algebra $O_{q}\left(S L_{2}\right)$ of the quantized $S L_{2}$, in which the elements $a, b, c, d$ given in the example are the standard generators; the antipode of this $O_{q}\left(S L_{2}\right)$ is bijective. See [21], for example.

\section{Non-uniqueness of "Picard-Vessiot rings"}

To see the non-uniqueness mentioned at the last paragraph of Example 16.1 we start with reproducing the definition of Picard-Vessiot rings adopted by Theorem 10.7, in a generalized situation. Let $\mathcal{H}$ be a Hopf algebra, and let $M$ be a finite-dimensional left $\mathcal{H}$-module. We say that a left $\mathcal{H}$-module algebra $\mathcal{R}$ is a Picard-Vessiot ring for $M$ (or for the corresponding matrix representation), if

(1) $\mathcal{R}$ is generated by the entries of $\mathbf{Y}^{ \pm 1}$, where $\mathbf{Y}$ is a matrix of fundamental system of solutions for $M$, 
(2) $\mathcal{R}$ is simple as an $\mathcal{H}$-module algebra, and

(3) there exists a $C$-algebra map $\mathcal{R} \rightarrow C$.

One sees that Condition (1) does not depend on the choice of a $C$-basis of $M$, since the matrix $\mathbf{Y}$ is not affected (up to conjugacy) by basis change. In the classical, commutative situation, Condition (3) is ensured by (1), if we assume, as usual, that $C$ is algebraically closed. But in the present, quantized situation, it seems reasonable to remove Condition (3), since the condition possibly exclude many non-commutative rings. We will see that the uniqueness does not hold any more if we remove the condition.

Let $\left(\mathcal{H}_{q}=\right) \mathcal{H}=C\left[s, s^{-1}\right] \otimes C[t]$ be the Hopf algebra which was defined by Definition 3.9 and reproduced in the preceding section. Note that the Hopf algebra $\mathcal{H}_{\pi}^{\circ}=C\left\langle e^{ \pm 1}, g\right\rangle$ obtained in Example 16.1 is isomorphic to $\mathcal{H}$; in fact, $s \mapsto e^{-1}$ and $t \mapsto e^{-1} g$ define an isomorphism

$$
\mathcal{H} \stackrel{\simeq}{\longrightarrow} \mathcal{H}_{\pi}^{\circ} .
$$

Since this $\mathcal{H}$ is pointed, every $\mathcal{H}$-torsor is necessarily cleft. It is known and easy to see that every $\mathcal{H}$-torsor is isomorphic to the trivial $\mathcal{H}$.

Assume that $q$ is a primitive $N$-th root of 1 , where $N>1$. Then the ideal $\mathcal{I}=\left(s^{N}-1, t^{N}\right)$ of $\mathcal{H}$ is a Hopf ideal. The quotient Hopf algebra $\mathcal{T}=\mathcal{H} / \mathcal{I}$ is called Taft's Hopf algebra; it is finite-dimensional and pointed. We denote the natural images of $s, t$ in $\mathcal{T}$ by the same symbols.

Example 17.1. - Given an element $\lambda \in C$, let $\mathcal{R}_{\lambda}$ denote the algebra generated by two elements $s^{\prime}, t^{\prime}$, and defined by the relations

$$
t^{\prime} s^{\prime}=q s^{\prime} t^{\prime}, \quad s^{\prime N}=1, \quad t^{\prime N}=\lambda .
$$

One sees that

$$
\rho\left(s^{\prime}\right)=s^{\prime} \otimes s, \quad \rho\left(t^{\prime}\right)=s^{\prime} \otimes t+t^{\prime} \otimes 1
$$

defines an algebra map $\rho: \mathcal{R}_{\lambda} \rightarrow \mathcal{R}_{\lambda} \otimes \mathcal{T}$, by which $\mathcal{R}_{\lambda}$ is a right $\mathcal{T}$-comodule algebra; it coincides with $\mathcal{T}$ if $\lambda=0$. It is essentially proved in [13] that $\mathcal{R}_{\lambda}$ is a (necessarily, cleft) $\mathcal{T}$-torsor, and $\mathcal{R}_{\lambda} \simeq \mathcal{R}_{\lambda^{\prime}}$ if and only if $\lambda=\lambda^{\prime}$. Moreover, if $C^{\times}=\left(C^{\times}\right)^{N}$, every $\mathcal{T}$-torsor is isomorphic to $\mathcal{R}_{\lambda}$ for some $\lambda$.

The same formulas as in (16.5) define a matrix representation $\pi$ of $\mathcal{T}$. Recall that $\mathcal{T}$ is a quotient Hopf algebra of $\mathcal{H}$. By the same argument as in Example 16.1, we see that $\mathcal{T}_{\pi}^{\circ}$ is the quotient Hopf algebra of $\mathcal{H}_{\pi}^{\circ}$ by the Hopf ideal $\left(e^{N}-1, g^{N}\right)$, and the isomorphism (17.1) induces an isomorphism $\mathcal{T} \simeq \mathcal{T}_{\pi}^{\circ}$. Moreover, the isomorphism (17.1) induces such an isomorphism that sends $\mathcal{R}_{\lambda}$ onto the quotient $\mathcal{R}_{\lambda}^{\prime}$ of $\mathcal{H}_{\pi}^{\circ}$ by the ideal $\left(e^{N}-1, g^{N}-\lambda\right)$; this $\mathcal{R}_{\lambda}^{\prime}$ is thus a $\mathcal{T}_{\pi}^{\circ}$-torsor. Let us identify $\mathcal{R}_{\lambda}$ with $\mathcal{R}_{\lambda}^{\prime}$ through the induced isomorphism. Then $\mathcal{R}_{\lambda}$ turns into a left $\mathcal{T}$-module algebra which is simple 
by Proposition 14.3. Let $e^{\prime}, g^{\prime}$ denote the natural images of the elements $e$, $g$ of $\mathcal{H}_{\pi}^{\circ}$ in $\mathcal{R}_{\lambda}$. In view of (16.6), we see that

$$
\left[\begin{array}{ll}
e^{\prime} & 0 \\
g^{\prime} & 1
\end{array}\right]
$$

is a matrix of fundamental system of solutions for $\pi$. Apparently, the entries already generate $\mathcal{R}_{\lambda}$.

To summarize, $\mathcal{R}_{\lambda}, \lambda \in C$, are mutually non-isomorphic $\mathcal{T}$-module algebras satisfying Conditions (1) and (2) above. Note that $\mathcal{R}_{\lambda}$ satisfies Condition (3) if and only if $\lambda=0$.

\section{Acknowledgments}

The authors would like to thank the referees for their helpful suggestions and warm support.

\section{Bibliography}

[1] K. Amano, A. Masuoka \& M. TAkeuchi, "Hopf algebraic approach to PicardVessiot theory", vol. 6, p. 127-171, Elsevier; North-Holland, 2009.

[2] Y. AndRÉ, "Différentielles non commutatives et théorie de Galois différentielle ou aux différences. (French) [Noncommutative differentials and Galois theory for differential or difference equations]", Ann. Sci. Éc. Norm. Supér. 34 (2001), p. 685-739.

[3] P. CARtier, "Personal correspondence", November 2013.

[4] L. Euler, Introductio in Analysin Infinitorum, Tomus Primus, Springer, 1992, Seies mathematica volumen VIII, Opera Omnia of L.Euler. Original book was published in 1748 .

[5] C. Hardouin, "Iterative difference Galois theory", J. Reine Angew. Math. 644 (2010), p. 101-144.

[6] H. Haynal, "PI degree parity in $q$-skew polynomial rings", J. Algebra 319 (2008), no. 10, p. 4199-4221.

[7] F. Heiderich, "Galois theory of module fields", PhD Thesis, Barcelona University, 2010.

[8] E. Heine, "Über die Reihe $1+\frac{\left(q^{\alpha}-1\right)\left(q^{\beta}-1\right)}{\left(q^{\gamma}-1\right)(q-1)} x+\frac{\left(q^{\alpha}-1\right)\left(q^{\alpha+1}-1\right)\left(q^{\beta}-1\right)\left(q^{\beta+1}-1\right)}{\left(q^{\gamma}-1\right)\left(q^{\gamma+1}-1\right)(q-1)\left(q^{2}-1\right)} x^{2}+$ ...", J. Reine Angew. Math. 32 (1846), p. 210-212.

[9] C. G. J. JACOBI, Fundamenta nova theoriae functionum ellipticorum, Cambridge University Press, 2013, Reprint of the paper published in Königsberg 1829.

[10] S. MAJID, Foundation of quantum group theory, Cambridge University Press, 1995.

[11] B. Malgrange, "Le groupoïde de Galois d'un feuilletage", in Essays on geometry and related topics, Vol. 1, 2, Monographies de l'Enseignement Mathématique, L'Enseignement Mathématique, 2001, p. 465-501.

[12] Y. I. Manin, Quantum groups and noncommutative geometry, Centre de Recherches Mathématiques de l'université de Montréal, 1988. 
[13] A. MASUOKA, "Cleft extensions for a Hopf algebra generated by a nearly primitive element", Commun. Algebra 22 (1994), p. 4537-4559.

[14] A. Masuoka \& M. YANAGAWA, " $\times_{R}$-bialgebras associated with iterative $q$-difference rings", Int. J. Math. 24 (2013), no. 4, article no. 1350030 (27 pages).

[15] S. Montgomery, Hopf algebras and their actions on rings, Regional Conference Series in Mathematics, vol. 82, American Mathematical Society, 1993.

[16] S. Morikawa, "On a general difference Galois theory. I", Ann. Inst. Fourier 59 (2009), p. 2709-2732.

[17] S. Morikawa \& H. Umemura, "On a general difference Galois theory. II", Ann. Inst. Fourier 59 (2009), p. 2733-2771.

[18] M. NoumI, "Quantum Grassmannians and $q$-hypergeometric functions", $C W I$ Q. 5 (1992), no. 4, p. 293-307.

[19] M. VAn Der Put \& M. F. Singer, Galois theory of linear differential equations, Springer, 2003.

[20] M. Sweedler, Hopf algebras, Mathematics Lecture Series, W.A. Benjamin, 1969.

[21] M. TAKEuCHI, "Hopf algebra techniques applied to the quantum group $U_{q}\left(s l_{2}\right)$ ", in Deformation theory and quantum groups with applications to mathematical physics, Contemporary Mathematics, vol. 134, American Mathematical Society, 1992, p. 309323.

[22] H. Umemura, "Differential Galois theory of infinite dimension", Nagoya Math. J. 144 (1996), p. 59-135.

[23] - "Galois theory of algebraic and differential equations", Nagoya Math. J. 144 (1996), p. 1-58.

[24] - "Galois theory and Painlevé equations", in Théories asymptotiques et équations de Painlevé, Séminaires et Congrès, vol. 14, Société Mathématique de France, 2006, p. 299-339.

[25] - "Invitation to Galois theory", in Differential equations and quantum groups, IRMA Lectures in Mathematics and Theoretical Physics, vol. 9, European Mathematical Society, 2007, p. 269-289.

[26] _ "Picard-Vessiot theory in general Galois theory", vol. 94, p. 263-293, Polish Academy of Sciences, 2011. 